\title{
Characteristics of Horizontal Liquid - Liquid Flows in a Circular Pipe using Simultaneous High-Speed Laser-Induced Fluorescence and Particle Velocimetry
}

Rhys G. Morgan ${ }^{\mathrm{a}}$, Christos N. Markides ${ }^{\mathrm{b},{ }^{*}}$, Ivan Zadrazil ${ }^{\mathrm{c}}$, Geoffrey F. Hewitt ${ }^{\mathrm{d}}$

Department of Chemical Engineering, Imperial College London, London SW7 2AZ, UK

\footnotetext{
rhys.morgan@imperial.ac.uk

${ }^{b}$ c.markides@imperial.ac.uk

${ }^{c}$ i.zadrazil06@imperial.ac.uk

${ }^{d}$ g.hewitt@imperial.ac.uk
}

\begin{abstract}
This paper describes a set of experiments on liquid-liquid flows in a horizontal circular tube. The liquids used in the experiments were an aliphatic hydrocarbon oil (Exxsol D80) and an aqueous solution of glycerol. The concentration of glycerol in the solution was adjusted so that the two liquids had the same refractive index, and optical distortions due to the curvature of the (transparent) circular tube test section were corrected for with the use of a graticule technique. The test section was far downstream of an inlet section that established an initially stratified co-current flow of the two immiscible liquids, with the Exxsol D80 oil flowing over the glycerol/water solution. The flows were investigated at the test section with the application of laser-based optical diagnostic methods, which included high-speed simultaneous Planar Laser Induced Fluorescence (PLIF), Particle Image Velocimetry (PIV) and Particle Tracking Velocimetry (PTV). These techniques allowed the reliable evaluation of the nature of the investigated

\footnotetext{
${ }^{*}$ Corresponding author. Tel.: +44 2075941601.

E-mail address: c.markides@imperial.ac.uk (C. N. Markides).
} 
horizontal liquid-liquid flows (i.e., the flow patterns from phase distribution information), together with the detailed spatiotemporally resolved measurement of key flow characteristics such as phase and velocity distributions, and also of important parameters such as droplet size. The resulting PLIF images provide a clear indication of the distribution of the phases within a plane that passed through the channel centreline, and are used to obtain qualitative information about the arising flow patterns. The images were also used quantitatively to generate data on phase distribution, in-situ phase fraction, interface level and droplet size distribution. Much of the PLIF data on in-situ phase fraction and interface level agrees well with predictions from a simple stratified laminar-laminar flow model. The particle velocimetry methods (PIV and PTV) provide data on the velocity profiles in the investigated flows. Over the range of superficial velocity conditions investigated, the velocity profile in the lower (heavier and more viscous) glycerol/water solution phase was typically characteristic of laminar flow, whereas in the upper (lighter and less viscous) Exxsol D80 oil phase the profile often showed a shape characteristic of turbulent flow.

Keywords: Horizontal liquid-liquid flow; flow regimes; laser measurements; phase fraction; interface level; droplet size; velocity profile 


\section{Introduction}

The co-current flow of two immiscible liquids is encountered in wide variety of industrial applications. The investigation described here arose in the context of liquid-liquid flows in subsea pipelines in petroleum production facilities, where the fluids are oil and water. The water can either occur naturally in the reservoir (this is known as "connate water") or result from water injection into the reservoir to increase pressure and in turn enhance oil recovery (EOR).

An ability to characterise liquid-liquid flow behaviour accurately is of fundamental importance. For example, the accurate prediction of the in-situ phase fraction in two-phase flows allows the determination of numerous other flow parameters, such as the two-phase density and viscosity, which are key requirements for the closure of multiphase models for predicting the flow behaviour, particularly the pressure drop and flow pattern transitions, both of which are dependent on the in-situ phase fraction.

The importance of liquid-liquid flows led to a number of studies of such flows (see for example Russell and Charles, 1959; Arirachakaran, et al., 1989; Solemani, 1999). However, these studies concentrated on measurement of overall parameters such as pressure gradient and phase holdup; the objective of the study described here was to obtain much more detailed information about the liquid-liquid flow behaviour using modern optical techniques. By matching the refractive index of the two liquids and adding a fluorescent dyestuff to one of the phases, it is possible to determine the phase distribution using planar laser-induced fluorescence (PLIF). Liu (2005) reports a study of vertical downflow of liquid-liquid flow mixtures using PLIF and, more recently, Morgan, et al. (2012) reported the application of the technique to horizontal liquid-liquid flows. Though the studies of Liu (2005) and Morgan, et al. (2012) revealed a number of interesting new phenomena, they were both carried out with channels which had a square cross-section in order to avoid optical distortion of the image by the curved (transparent) tube wall; this is clearly not typical of the real applications in which tubes of circular cross-section are the norm. In the present study, circular tubes were used together with an automated method for correcting the images for distortion.

One of the most powerful tools for the study of fluid flow and mixing is laser-induced fluorescence (LIF). Liu (2005) employed PLIF to visualise co-current liquid-liquid downward flows and produced images 
with a strong and clear distinction between the two phases. Liu (2005) used these images to study the phase inversion phenomena that arose in the investigated flows and found an ambivalent range over which the identity of the continuous phase alternates in time between the one fluid and the other. This was extended to the more practical case of horizontal liquid-liquid flows in an original study by the present authors (Morgan, et al., 2012). However, phase information on its own lacks the ability to provide a full insight into the numerous facets of these multiphase flows. The current work is a development of that described in Morgan, et al. (2012), not only in the use of a circular cross-section tube (a square cross-section tube was used in the work described in Morgan, et al. (2012), and also in the work by Liu (2005)), but also in the extension of the laser measurement techniques to include, in addition to PLIF, Particle Image Velocimetry (PIV) and Particle Tracking Velocimetry (PTV). To the best of the author's knowledge, the results described in the present paper are the first obtained in liquid-liquid systems using simultaneous PLIF and PIV/PTV. This enhanced measurement capability, and specifically the use of PIV/PTV, enables the detailed diagnostic inspection of the co-current liquid-liquid flow velocity profiles. In addition, the use of a tube with a circular cross-section is more representative of industrial pipeline systems, although it is optically more challenging. The provision of detailed data in the circular pipe section has been made feasible by the utilisation of an image correction technique involving the use of a graticule (printed target screen), as described in detail in Sections 2.3.1.

The analysis performed on the data (i.e., images) generated by the laser-based techniques, and the associated results presented in this paper, are similar to those used to characterise the square section flows presented in Morgan, et al. (2012). However, herein the quantitative analysis is extended to include results for the velocity profiles in the flow. A qualitative analysis of the results, including images of the flow regimes observed and a flow regime map constructed from the flow regime classifications, is presented first in Section 3.1. Following this, the subsequent sections present the results from the quantitative analyses of the flow images, as follows: (i) vertical phase distribution profiles (in Section 3.2); (ii) in-situ phase fractions (in Section 3.3); (iii) interface level data (in Section 3.4); (iv) droplet size distribution results (in Section 3.5), and; (v) velocity profiles (in Section 3.6). Finally, the main conclusions from this work can be found in Section 4. 


\section{Experimental Methods}

This section presents the experimental flow facility, test liquids, measurement procedure and postprocessing analysis, and includes a description of the apparatus (inlet, main test section, visualisation section), optical measurements (laser system, camera, synchronisation system) and the image processing methodology used in the present study. Specifically, the flow facility, related experimental procedures and flow conditions are presented in Section 2.1. Section 2.2 discusses the rationale behind the selection of the two test fluids (based on refractive index matching), along with a presentation of the equipment and techniques employed for the optical flow measurements, including a detailed description of the circular cross-section visualisation section. Finally, a description of the image processing, data analysis and related calculations performed on the raw images generated from the laser techniques in order to obtain the final results is given in Section 2.3.

\subsection{Flow facility, procedure and conditions}

\subsubsection{Flow facility and experimental procedure}

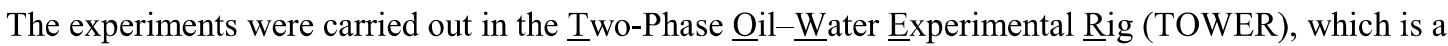
multiphase flow facility at Imperial College London. This facility was designed and built for the detailed investigation of flow phenomena occurring in co-current horizontal liquid-liquid flows. A schematic diagram of the facility is shown in Figure 1. The main test section of the rig comprises a $7.30 \mathrm{~m}$ long and 1-inch $(D=25.4 \mathrm{~mm})$ nominal bore stainless steel circular pipe. The same facility was also used in our earlier related study with a square section visualisation section (Morgan, et al., 2012), making comparisons between the two sets of results highly appropriate.

The test fluids used in the present effort are also the same as those used in the earlier study by Morgan, et al., (2012), namely: (i) Exxsol D80 oil, a kerosene-like aliphatic hydrocarbon, and; (ii) a homogeneously mixed solution of glycerol in water. Details of the test fluids and their selection can be found in Section 2.2.2. The flows were provided by two Grundfos CRN 10-5 pumps with three-phase supplies, 
one for each fluid. The pumps each have a maximum rated flow-rate of $2.8 \mathrm{~L} . \mathrm{s}^{-1}$ and a maximum rated pressure of 3.60 bar. The flow-rates were measured by means of four NB liquid turbine flow-meters, fitted with a Fluid Well FllQ-X LCD digital display. The flow-rates were time-logged onto a computer by means of a 4-20 mA linear current output. Each fluid was directed through one of two turbine flowmeters. The orientation of this arrangement is illustrated schematically in Figure 1. The two flow-meters for each fluid have different measurement flow-rate ranges, these being: $2-20 \mathrm{~L} \cdot \mathrm{min}^{-1}$ and $14-$ $140 \mathrm{~L} \cdot \mathrm{min}^{-1}$, denoted by $\mathrm{FM}_{\text {low }}$ and $\mathrm{FM}_{\text {high }}$ respectively in Figure 1. The reason for adopting this approach is that it facilitates experimental runs to be conducted over a broader range of flow-rates and phase fractions, while minimising the overall experimental uncertainty in the setting and measurement of the flow-rates. The accuracy of the NB liquid flow turbines (both $\mathrm{FM}_{\text {low }}$ and $\mathrm{FM}_{\text {high }}$ in Figure 1) is $\pm 0.5 \%$, while their repeatability is $\pm 0.1 \%$ of full scale.

The inlet to the test section is configured such that either of the test fluids can be introduced into the test section on top of the other. In the experimental campaign detailed in this paper the oil phase was introduced on top of the glycerol/water solution phase. In addition, a $1.5 \mathrm{~mm} \mathrm{~K}$-type thermocouple, positioned directly before the inlet to the test section, was used to monitor the temperature of the flow. In the experimental campaign described in this paper the temperatures of the flows were within a maximum deviation of $2{ }^{\circ} \mathrm{C}$ from ambient, i.e., $21^{\circ} \mathrm{C}$.

\subsubsection{Flow conditions}

The flow conditions are defined via two independent parameters: (i) the superficial mixture velocity $U_{\mathrm{m}}$, defined as the total volumetric flow-rate of both liquids (oil and aqueous glycerol) in the pipe section $Q_{\mathrm{T}}=Q_{\mathrm{oil}}+Q_{\mathrm{gs}}$ divided by the cross-sectional area of the pipe $A=\pi D^{2} / 4$, and; (ii) the inlet (volumetric) phase fraction of oil in the pipe $\phi_{\text {in }}$, defined as the volumetric flow-rate of oil $Q_{\text {oil }}$ divided by the total volumetric flow-rate of both liquids $Q_{\mathrm{T}}$ at the inlet of the pipe section. The two independent flow parameters $U_{\mathrm{m}}$ and $\phi_{\mathrm{in}}$ were varied independently in 48 test runs. The two parameters were kept constant for the duration of each run (i.e., set of conditions). The parameters were set by the volumetric flow-rates of each phase (i.e., the oil $Q_{\mathrm{oil}}$ and aqueous glycerol $Q_{\mathrm{gs}}$ ) being fed into the pipe at the inlet that were 
adjusted by means of suitable valves. The investigated experimental conditions spanned a range of $U_{\mathrm{m}}$ from 0.11 to $0.84 \mathrm{~m} \cdot \mathrm{s}^{-1}$ and $\phi_{\text {in }}$ from 0.1 to 0.9 . The aim has been to focus on the flow regime transition from stratified to dual continuous flow, in order to enhance our understanding of the flow characteristics in the regimes between stratified and dispersed flow. The matrix of experimental conditions was selected based upon consultation of previous flow regime maps, most notably those by Soleimani (1999) and Hussain (2004), and is largely the same as that in Morgan, et al. (2012).

\subsection{Optical methods and test fluids}

\subsubsection{Optical measurement techniques}

A multitude of optical diagnostic techniques for non-intrusive flow visualisation and/or measurement can be adopted for the study of multiphase flows (e.g., Hewitt, et al., 1990; Barbosa, et al., 2001; Liu, 2005). Of these, there are two main categories: optical methods and tracer methods, of which PLIF, as employed in the current investigation, falls into the former category, whereas PIV and PTV both fall into the latter.

PLIF is a spectroscopic technique that was applied in the present work to visualise the complex interfacial configurations which occur in the flow of two immiscible liquids. In PLIF it is necessary to provide a means of distinguishing between one liquid phase and the other within the plane of illumination, and this was achieved in the present experiments by adding a fluorescent dyestuff (Eosin $\mathrm{Y}$ ) to the aqueous glycerol solution (see Section 2.2.2). Eosin Y emits light (fluoresces) following excitation by the laser light sheet. This allows an unequivocal identification of the aqueous glycerol phase, and consequently of the position of the interfaces between the phases. By using high-speed imaging (see Section 2.2.4), the temporal development of the interfacial structure in the illumination plane was achieved.

A key requirement in the application of this technique was the matching of the refractive indices of the two liquids. This allowed the viewing of a fixed illuminated plane through the flow, without distortions of the incident laser sheet, and also eliminated distortions of the collected light in the line-of-sight of the camera. In both cases the distortions would arise due to changes in the refractive index within the flow. Because, in the experiments described here, the test section tube was circular, matching the refractive 
indices is a necessary but not sufficient requirement to eliminate the effects of distortion. It was necessary to further correct the images to account for the effect of the tube and the methodology used for achieving this is described in Section 2.3.1 below.

PIV and PTV are optical methods for the measurement of velocity fields based on either elastic Mie scattering (when particles with diameters of the order of the wavelength of the incident light are used) or geometric reflection (when particles with diameters an order of magnitude, or more, greater than the incident light wavelength). In our experiments these techniques were used to measure the axial and radial components of velocity within a 2-dimensional illuminated plane normal to the flow axis. The laser light source used to create the sheet for the illumination of this plane had a wavelength of $510.6 \mathrm{~nm}$ (see Section 2.2.4), while the particles (in fact, micro-bubbles and micro-droplets; refer to Section 2.3.7) used for the PIV/PTV analysis were selected to be of the order of a few tens to $100 \mu \mathrm{m}$. Both techniques can be used to provide instantaneous vector maps of flow velocity within the illuminated flow plane. The main difference between PIV and PTV concerns the recovery of velocity information from correlations of the positions of groups of particles within defined windows (in the case of PIV) or, correlations of individual particle positions (the case of PTV), between successive images. The ability to use these techniques depends on the density of the seeded particles in the flow; with optimal PIV requiring higher densities than PTV. In the present work the density of the micro-bubbles and micro-droplets was such that it was possible to apply PIV, in a first stage of processing, in order to assess reference vectors, followed by PTV in a second stage of processing. Pairs of successive images are taken with a small delay that depends on the flow velocity (and in the case of PIV, the size of the PIV window). The correlation calculations are done by post-processing algorithms for the recovery of velocity vector maps.

Accurate optical PLIF and PIV/PTV measurements demand a fixed and known illumination plane, a lack of distortions within the imaging line-of-sight (or an ability to correct reliably for these distortions), a clear distinction between the two fluids and a clear and well-timed observation of particles (here, microbubbles) that faithfully follow the surrounding flow. A number of key factors have to be accounted for in order to achieve this. These factors largely pertain to: (i) the experimental arrangement (flow geometry, test fluids, optical paths); (ii) the choice of the selected light source (beam energy and excitation wavelength), and; (iii) the choice of the fluorescent compounds and tracers. So as to ensure improved 
measurement quality, this study employs two fluids with matched refractive indices, as well as a specially constructed visualisation section at the immediate location where the laser measurements were performed, whose purpose was to reduce laser light intensity non-uniformity and image distortion in the measurement plane due to the curved walls of the containing circular duct. The selection of the fluids and dyes is discussed in Section 2.2.2, while the visualisation section is presented in Section 2.2.3. Finally, information on the laser system is provided in Section 2.2.4.

\subsubsection{Test fluid selection}

As mentioned previously, the two immiscible liquids employed are a kerosene-like aliphatic hydrocarbon, specifically Exxsol D80 oil, and a homogeneously mixed solution of glycerol in water. The two liquids are the same as those used in our earlier study of horizontal liquid-liquid flows in a square duct (Morgan, et al., 2012), while the oil has identical physical properties to the oils used by Soleimani (1999), Hussain (2004), and Liu (2005), and similar property values to those used by Angeli (1996), and, Angeli and Hewitt (2000). The density ratio of the test fluids (aqueous glycerol solution to oil) is 1.5 , which is comparable to the density ratios found in many oil-water systems, usually in the range 1 to 1.5 (Russell, et al., 1959; Charles and Lilleleht, 1966; Simmons and Azzopardi, 2001; Ioannou, et al., 2005). In addition, the fluids have a viscosity ratio (aqueous glycerol solution to oil) of approximately 20 , which is comparable to the viscosity ratios found in the work by Charles and Lilleleht (1966), and Guzhov, et al. (1973). However, in much of the previous experimental work the oil is the less dense and more viscous fluid, while in the current study the oil is the less dense and also the less viscous fluid.

In order to obtain clear and accurate data from the planar laser-based imaging techniques, any out-ofplane refraction, reflection or scattering into the camera of the incident light needs to be eliminated as much as possible. This allows the successive illumination of a known and invariant measurement (interrogation) plane by the incident laser sheet and also an undistorted line-of-sight image of the light coming from the measurement plane onto the camera. In the current study this was achieved by the use of a specially designed visualisation section featuring flat outside windows for light delivery and imaging, (as described in detail in Section 2.2.3) and by having refractive index matched liquids that were 
transparent to both the incident light (excitation peak at $510.6 \mathrm{~nm}$ ) and the generated broadband fluorescence light (with an emission peak at $544 \mathrm{~nm}$ ). The refractive indices were measured using an Abbe 60 Refractometer manufactured by Bellingham and Stanley. A solution of $80 \%$ by weight glycerol in $20 \%$ water at $20^{\circ} \mathrm{C}$ was found to match the refractive index of Exxsol D80 oil to 3 decimal points, with a refractive index of 1.444 .

The composition of the final glycerol/water-dye solution (glycerine, water and seeded fluorescent dye mix) was optimised to maximise the brightness of the fluorescent phase and hence the contrast between the phases, while minimising the absorption of incident light by the added dye. This required the addition (seeding) of $0.4 \mathrm{~mL}$ of fluorescent dye solution (containing $5 \%$ by weight Eosin $\mathrm{Y}$ dye in $95 \%$ water) to each litre of aqueous glycerol solution, i.e., $0.4 \times 10^{-3}(\mathrm{v} / \mathrm{v})$. The resulting mass fraction of pure dye in the glycerol/water-dye mixture is $1.7 \times 10^{-5}$ and the corresponding molar fraction is $1.4 \times 10^{-5}$, to 2 significant figures. The absorption and emission peaks of the Eosin Y fluorescent dye are found at $524.8 \mathrm{~nm}$ and $544 \mathrm{~nm}$, respectively, though the emitted fluorescence spectrum is broadband, such that the fluorescence emission signal drops to $10 \%$ of its peak value at about $630 \mathrm{~nm}$ and $1 \%$ of its peak value at about $710 \mathrm{~nm}(\mathrm{Du}$, et al., 1998). The physical properties of the two selected liquids are presented in Table 1.

Direct measurements were made of the interfacial tension between the glycerol/water solution and oil phases at varying concentrations of Eosin $\mathrm{Y}$ dye in the glycerol/water solution phase. This was done in order to ascertain whether, and if so to what degree, the addition of the Eosin Y dye (whose addition was necessary for the fluorescence measurements) affected this important property of the two-phase system. A significant modification of the interfacial tension can lead to altered observations of interfacial phenomena and, consequently, a non-representative analysis of the system. The results from these measurements were presented in Morgan, et al. (2012), and showed that the interfacial tension is not altered significantly from its value at zero dye concentration, making the present results directly relevant and transferable to generic two-phase oil-water systems.

Finally, it is noted that the two liquids under investigation are immiscible and that there is no diffusion of one phase into the other, something that is confirmed by the measurements. As such, the purpose here is 
not the recovery of instantaneous concentration information that would be necessary in diffusive flows (as done, for example, by Markides and Mastorakos (2006)). In the current flows the normalised concentration of the two phases is at all times either locally zero or unity. Instead, the key measurement of interest relates to the correct identification of the interface between the phases, where this exists in the flow. The PLIF measurement methodology employed is only concerned with the on/off presence/absence of fluorescent dye, which (given that the dye is only soluble in the aqueous glycerol phase and insoluble in oil) signifies the local, unique presence of the aqueous glycerol solution. Details of the procedure used to determine the local presence/absence of the aqueous glycerol phase are given below in Section 2.3.2.

\subsubsection{Circular Cross-Section Visualisation Section}

Although the data generated with the square cross-section duct were useful in the general understanding of these flows (Morgan, et al., 2012), it is obvious that a duct of circular cross-section would be more representative of industrial pipeline systems. A problem that arises is that of image distortion by the (circular) walls of the flow tube. This problem was addressed by identifying a solid transparent material with a refractive index that is identical to the (matched) refractive indices of the liquids, and constructing the (circular cross-section) observation/measurement section from this material. Considerable effort was placed on establishing a triply refractive index matched system, i.e., one in which both test fluids and the pipeline wall material have the same refractive indices. However, this task is particularly challenging due to the added constraint of establishing a wall material of suitable characteristics, i.e., transparent to both the laser light and the fluorescence light, sufficient strength and rigidity, and chemically compatible with the test fluids. It was found that the material most closely matching these requirements was borosilicate glass; however, the refractive index of this material is 1.474 , which although being a close match to that of the fluids used in the square cross-section duct studies (1.444), was not a perfect match.

The alternative approach was to accept that the images produced would be distorted to a small (but, constant) degree due to the small (and constant) mismatch between the refractive index of the fluids and that of the tube walls, and to correct for this distortion using an image processing technique. As a precursor to the experiments, a graticule with a printed target of known dimensions was mounted inside 
the visualisation pipe section such that its flat surface was in the same position as that traversed by the planar laser sheet. The known positions of points on the graticule could then be related to their apparent position in the generated (distorted) images. This allowed the images to be corrected for the distortion. Details of the image correction method are given in Section 2.3.1.

The circular cross-section visualisation section adopted is shown in Figure 2 and consists of an $L_{\mathrm{S}}=100 \mathrm{~mm}$ long (or, 3.6 equivalent diameters, $L_{\mathrm{S}} / H_{\mathrm{T}}=3.6$ ) borosilicate glass pipe with an internal diameter of $H_{\mathrm{T}}=27.6 \mathrm{~mm}$. The visualisation section was housed within a Perspex box. The void between the pipe section and the internal walls of the box was filled with the test fluid that is not seeded with fluorescent dye (i.e., Exxsol D80). The section was located $L_{\mathrm{E}}=6.20 \mathrm{~m}$ downstream of the inlet of a horizontal $D=25.4 \mathrm{~mm}$ diameter stainless steel pipe (such that $L_{\mathrm{E}} / D=244$ ). The small difference in diameter between the stainless steel pipe and the test section tubing and the effects of this were minimised by arranging a smooth transition between the respective tubes. As was stated above, the difference in refractive index between the wall material and the fluids causes a distortion of the image; this distortion was minimised by the choice of wall material and the use of a planar fluid-filled volume outside the tube.

\subsubsection{Laser system, camera and synchronisation}

An Oxford Lasers LS20-10 pulsed copper vapour laser with a nominal output power of $20 \mathrm{~W}$ and an internal clock frequency of $10 \mathrm{kHz}$ was used as the green light source for the experimental campaign. The output light spectrum exhibits a peak at $510.6 \mathrm{~nm}$, has a pulse duration of $2 \mathrm{~ns}$ and a pulse energy of approximately $2 \mathrm{~mJ}$. Various pulse repetition frequencies can be achieved with the use of an external frequency source. The flows presented herein were recorded at either 1 or $2 \mathrm{kHz}$, depending on the flowrate (and thus, flow velocity). A dedicated light sheet generator produced by Oxford Lasers was connected to the copper vapour laser by means of a fibre optic cable. The resulting laser sheet had a thickness of less than $1 \mathrm{~mm}$. The arrangement of the laser light sheet and related details are similar to those described in detail in Morgan, et al., (2012).

The fluorescent images resulting from the laser illumination of the test section were video recorded using an iSpeed3 high-speed video cameras produced by Olympus. The camera has a maximum resolution of 
$1,280 \times 1,024$ pixels at which the maximum attainable frame-rate is $2,000 \mathrm{fps}$. The actual imaging frequency employed in the current measurements was set by the laser repetition frequency, which was either 1 or $2 \mathrm{kHz}$, as mentioned in the previous paragraph. A Macro $105 \mathrm{~mm}$ F2.8 EX DG medium telephoto lens produced by Sigma Imaging Ltd was used. The suitability of the lens was confirmed from evaluation of its MTF (Modulation Transfer Function) characteristic. The aperture was optimised to a setting of $f / 8$ (where $f$ is the focal length of the camera) to maximise image sharpness. The resulting spatial pixel resolution was $33 \mu \mathrm{m}$ per pixel.

The copper vapour laser delivers a pulsed output and it was necessary to synchronise this with the camera system to ensure that the laser produced pulses during the exposure of the camera and that the camera captured the resulting laser-induced fluorescence. A trigger box employing TTL (transistor-transistor logic) signals was used to synchronise the laser with the camera. The trigger box was driven by a framerate signal outputted from the camera. From the trigger box a signal was then sent to the laser. The signal reduced the pulse repetition frequency of the laser from its internal clock frequency of $10 \mathrm{kHz}$ to the frequency the camera was set to, for as long as the camera was allowed to capture images.

\subsection{Image Processing}

\subsubsection{Graticule Correction Technique}

The graticule calibration target (see Figure 3) contains a flat planar surface on to which crosses of known size and spacing were printed. During the calibration this surface was orientated so that it aligned with the central vertical plane of the visualisation section; i.e., the same plane as that which the laser light sheet penetrated the visualisation section during normal measurement operation. It should be noted that during the calibration no laser light was used; only ambient light was used to illuminate the visualisation section.

Prior to, and after each set of experimental runs the graticule calibration piece was inserted into the visualisation section. This was done by detaching the downstream connection between the visualisation section and the main pipeline. Once the graticule had been inserted, the test section was filled with Exxsol D80. Considerable care was taken to immobilise the test section and the adjoining visualisation 
section (see Figure 2) during this process. A limit of 1 pixel was imposed on the movement of the visualisation section when compared with the calibration images. As the size and spacing of the crosses on the graticule are known, one can use the values to measure the displacement and distortion of them when it is imaged by the camera through the visualisation section. Figure 3(a) shows a raw image of the graticule when viewed through the visualisation section. Based on the discrepancy between the generated image and the known position of the crosses, one can determine the manipulation that needs to be performed on the image of the graticule to restore the crosses to their actual size and spacing. The operation was performed using existing algorithms in the DaVis software package produced by LaVision. The resulting correction was based upon the average of 500 instantaneous images. Figure 3(b) shows Figure 3(a) after it has undergone correction. Since all of the images of the liquid-liquid flows obtained using the laser-based techniques undergo the same distortion, the same manipulation as that used for the graticule images can be applied to all of the flow images to remove the distortion.

Figure 3(c) shows an example of a raw image and Figure 3(d) shows the corresponding image after the correction has been undertaken. Though the correction is a necessary one, it is noted that the distortion is small; this reflects the small discrepancy in the refractive index of the liquids and that of the solid material of choice for the walls of the visualisation section (i.e., borosilicate glass), as well as the practice of filling a compartment with a flat outer wall, surrounding the visualisation section, with Exxsol D80.

\subsubsection{LIF image processing}

The phase distribution analysis, the results of which are presented in Section 3.2, has been performed by first converting the PLIF images of the flow into binarised black and white equivalents $I(x, y, t) \equiv I(x, y)_{i}(I$ taking a value between zero and unity depending on the local, instantaneous phase) by using a thresholding approach discussed in detail in Morgan, et al. (2012), and then processing the pixel information of the resulting image $I(x, y, t) \equiv I(x, y)_{i}$ using an in-house code in MATLAB. The threshold value was chosen as a compromise between smaller values that were more sensitive in identifying the exact location of the interface and larger values which were less sensitive to noise. The resulting relative (systematic) uncertainty that is introduced by the final choice of the threshold value in the result for the 
interface locations and consequently the instantaneous vertical phase fraction profiles has been estimated from direct measurements to be about $\pm 10 \%$ at a $95 \%$ confidence level, with a corresponding (systematic) uncertainty in the result for the instantaneous phase fraction of $\pm 4 \%$. The result from the procedure was an image with dark regions representing the oil phase and white regions representing the glycerol/water solution phase (which contained the fluorescent dye).

All of the data has been taken along the centreline of the circular cross-section duct. A key assumption of the phase fraction analysis that has been conducted (i.e. the phase distribution profiles and the in-situ phase fraction) is that there is a flat interface between the phases. The validity of this assumption has been assessed using work by $\mathrm{Ng}$ (2002). This revealed the interface to be flat along the cross-section of the pipe, to within close proximity of the wall.

Based on the binarised PLIF images $I(x, y, t) \equiv I(x, y)_{i}$, instantaneous and time-averaged oil phase fraction profiles (Section 2.3.3), in-situ phase fractions (Section 2.3.4), oil-glycerol/water solution interface levels (Section 2.3.5) and droplet sizes (Section 2.3.6) were evaluated. The approach employed was identical to that described by Morgan, et al. (2012), which allows a direct comparison of the two sets of results.

\subsubsection{Vertical oil phase fraction profiles}

The instantaneous phase fraction profile in the $i^{\text {th }}$ image, taken at time $t$, is denoted by $\phi(y, t) \equiv \phi(y)_{i}$, where $y$ is the height inside the visualisation section from the bottom of the pipe along a vertical axis perpendicular to the flow direction and pipe orientation, and $\phi$ is the corresponding phase fraction evaluated at that height over the length available in that image $L_{x}$ in the horizontal/flow direction $x$,

$$
\phi(y, t)=\frac{1}{L_{x}} \int_{x=0}^{L_{x}} I(x, y)_{i} d x
$$

The vertical phase fraction profiles $\bar{\phi}(y)$ reported in this paper refer to aggregated (i.e., time-averaged) phase profiles over a number of instantaneous images $n$ for a given flow condition, 


$$
\bar{\phi}(y)=\frac{1}{n} \sum_{i=1}^{n} \phi(y)_{i} .
$$

At least 1,000 frames were used to evaluate the time-averaged vertical profile of (oil) phase fraction $\bar{\phi}(y)$ from Eq. (2) for each condition. As with all other flow parameters calculated from the raw images, the number of pixels was converted to a length by using the known dimensions of the height of visualisation section. The phase fraction profiles are presented in Section 3.2. The relative experimental uncertainty in the estimation of the time-averaged profiles amounts to less than $\pm 1 \%$ at a $95 \%$ confidence level.

\subsubsection{In-situ phase fraction}

The instantaneous in-situ oil phase fraction $\langle\phi\rangle_{y}(t) \equiv\langle\phi\rangle_{y_{i}}$ in a single image is defined as the measured instantaneous volumetric ratio of oil, which is equal to the fraction of dark area in a single image. Thus, it is noted that the in-situ oil phase fraction $\langle\phi\rangle_{y, t}$ is an area fraction, unlike the input oil phase fraction $\varphi_{\text {in }}$ which is a volumetric flow-rate fraction. The in-situ phase fraction data $\langle\phi\rangle_{y, t}$ has been acquired by employing the same initial steps as those detailed for the phase distribution analysis, and then by timeaveraging the resulting vertical phase profiles to obtain a mean oil content in the measurement section for each flow condition, such that from $n$ images:

$$
\begin{aligned}
& \langle\phi\rangle_{y}(t)=\frac{4}{\pi H_{T}^{2}} \int_{y=0}^{y=H_{\mathrm{T}}} \phi(y, t) \sqrt{H_{T}^{2}-\left(H_{T}-2 y\right)^{2}} d y, \\
& \langle\phi\rangle_{y, t}=\frac{1}{n} \sum_{i=1}^{n}\langle\phi\rangle_{y_{i}} .
\end{aligned}
$$

The in-situ oil phase fraction data $\langle\phi\rangle_{y, t}$ are presented in Section 3.3. The uncertainty in the instantaneous in-situ oil phase fraction amounts to $\pm 4 \%$.

\subsubsection{Interface level}


The instantaneous interface level $H(x, t)$ is defined as the height of the oil-glycerol/water solution interface from the bottom of the pipe. The interface level analysis was performed by selecting thirty PLIF images from the recorded sequence. The selected images were 100 frames apart to ensure sample independence. The height $H$ was recorded at five points ( $x$ positions) in each image. Thus, for each flow condition 150 interface height values were generated. The experimental uncertainty in the instantaneous interface level $H(x, t)$ is the same as that for the instantaneous interface location, i.e., $\pm 10 \%$.

The mean $\mu_{H}$ (from Eq. (5)) and the standard deviation $\sigma_{H}$ (from Eq. (6)) of the oil-glycerol/water solution interface height $H$ data has been determined for each run (i.e., set of conditions):

$$
\begin{aligned}
& \mu_{H}=\frac{1}{n} \sum_{i=1}^{n} H_{i}, \\
& \sigma_{H}=\sqrt{\frac{1}{n-1} \sum_{i=1}^{n}\left(H_{i}-\mu_{H}\right)^{2}} .
\end{aligned}
$$

The results from the interface level analysis are presented in Section 3.4, where the mean interface levels $\mu_{H}$ are presented together with the $\mu+2 \sigma$ and $\mu-2 \sigma$ values. Assuming a normal distribution, these values represent the upper and lower limits of the $95 \%$ confidence interval. The resulting relative uncertainties in the statistical estimation of the mean $\mu_{H}$ and standard deviation $\sigma_{H}$ of the interface level height $H$ are both less than $\pm 1 \%(0.8 \%$ and $0.6 \%$, respectively).

\subsubsection{Droplet size}

Finally, a droplet size analysis was performed by measuring the area $A_{d}$ of a number of droplets as they appeared in the PLIF images, after converting the images to black and white binarised equivalents. For each set of conditions approximately 20 images and 100 droplets were selected, based on which Eqs. (7) and (8) were then used to evaluate a mean effective droplet diameter $\mu_{d}$ :

$$
d=2 \sqrt{A_{d} / \pi}
$$




$$
\mu_{d}=\frac{1}{n} \sum_{i=1}^{n} d_{i}
$$

The droplet size data can be found in Section 3.5. The relative uncertainty in the statistical estimation of the mean of the droplet size $d$ is $\pm 1 \%$.

Note that the mean effective droplet diameter that results from this calculation is in fact a mean droplet chord length. In Section 3.5 this is used to estimate a mean spherical droplet diameter, under the assumptions of uniformly spaced, spherical drops.

\subsubsection{PIV and PTV processing}

The velocity profiles within the oil and glycerol/water solution phases were evaluated by using the DaVis software package supplied by LaVision. Both the PIV and the PTV algorithms were applied to each data set. Although these particle velocimetry processes ordinarily require the presence of seeded particles, the determination of the velocity profiles was made possible in the present data from the presence of a broad range of micro-bubbles of air, and also of micro-droplets of one liquid phase in the other. The largest diameter of the micro-bubbles of air in our flows was $\sim 150 \mu \mathrm{m}$, by direct observation. Nevertheless, only micro-bubbles and micro-droplets of a certain size in the resulting images were selected for use in particle velocimetry computations; specifically, $2-5$ pixels in diameter. This was in order to prevent over sampling of individual micro-bubbles, and to ensure that these were small enough to closely follow the flow and low enough to eliminate "peak locking" quantization errors.

The PIV/PTV calculation procedure first involved the correction of the raw images for optical distortions, in a similar way to that used on the raw PLIF images, as described in Section 2.3.1; e.g., see Figure 4(a).

The corrected images were then pre-processed by an algorithm that subtracted off a sliding minimum over time, whereby the intensity of each pixel within a given image was compared with the intensity of the same pixel in the images immediately before it and after it, and the minimum value of the three was subtracted from that pixel. This improved the signal-to-noise ratio in the resulting images, i.e., the contrast between the "particles" (in this work, micro-bubbles or micro-droplets) and the background. A result of this procedure is shown in Figure 4(b). 
On completion of the pre-processing, the velocity vectors were then calculated via a multi-step PIV/PTV procedure in DaVis which employed a series of cross-correlation functions; firstly in a multi-pass PIV algorithm and finally a PTV algorithm. This process was performed on successive images, which were taken at 1 or $2 \mathrm{kHz}$, i.e., with a spacing of 0.5 or $1 \mathrm{~ms}$. Over this time, the typical displacement of the particles in the flows (recall that $U_{\mathrm{m}}=0.11-0.84 \mathrm{~m} \cdot \mathrm{s}^{-1}$ ) was between 3 and 20 pixels. In the first two PIV passes (the initial estimation of the velocity vectors), estimated velocity vectors within $128 \times 128$ pixel interrogation windows within the image were calculated. The third and final PIV pass was performed on $64 \times 64$ pixel PIV windows. An example of a velocity vector map calculated by the PIV algorithm is presented in Figure 4(c). Based on the intermediate PIV results, the final velocity vectors were calculated using a PTV approach in which individual particles within $8 \times 8$ pixel interrogation windows were tracked by employing the knowledge of the interrogation window displacement (from the intermediate velocity field) calculated previously by the PIV steps. Figure 4(d) presents an example of the instantaneous velocity vector map that was generated between two successive images.

Finally, for each given run, i.e., time-sequence of images, the instantaneous PTV velocity vector maps were time-averaged. Any obviously spurious vectors (i.e., vectors pointing in the opposite direction to the flow) within the time-averaged vector map were removed by employing a permissibility range on the velocity vectors, and in addition, the quality of the remaining vectors was further improved by applying a $3 \times 3$ median filter. The spurious vectors were either removed or replaced by a secondary or ternary cross-correlation peak. The resulting time-averaged PTV velocity vector map was then spatially averaged in the streamwise direction and filtered, which yielded a velocity profile (such as those presented in Figure 20). The resulting velocity profiles are presented in Section 3.6. 


\section{Results}

The PLIF and PIV/PTV measurements presented in this section have provided a wealth of new insights into the flow structure of liquid-liquid horizontal flows, as well as unique information concerning interfacial behaviour and the underlying velocity fields in the investigated flows. Characteristic images from the flow regimes observed are presented in Section 3.1, which also includes a flow regime map constructed from the observations. In the succeeding sections the results of the quantitative analysis of the generated data is presented. Specifically, Section 3.2 presents oil fraction distribution profiles along a vertical axis perpendicular to the flow centreline and Section 3.3 presents the results for time-averaged phase fraction. Sections 3.4 and 3.5 present the results for interface level and droplet size respectively. Finally, Section 3.6 presents the data on vertical velocity profiles.

The investigated flow conditions are defined via the two independent parameters defined in Section 2.1.2, namely: (i) the superficial mixture velocity $U_{\mathrm{m}}$, and; (ii) the inlet volumetric phase fraction of oil in the pipe $\phi_{\text {in }}$. These parameters were varied independently in 48 runs and kept constant for the duration of each run. The flow regime map that is presented in Section 3.1 has been constructed using the full matrix of experimental conditions up to superficial mixture velocities of $U_{\mathrm{m}}=0.84 \mathrm{~m} \cdot \mathrm{s}^{-1}$, whereas the quantitative analysis that follows in Sections 3.2 to 3.6 focuses on the lower superficial mixture velocities, in the range $U_{\mathrm{m}}=0.11$ to $0.42 \mathrm{~m} \cdot \mathrm{s}^{-1}$. In addition, $\phi_{\text {in }}$ was varied between 0.1 and 0.9 .

\subsection{Flow Phenomenology and Regime Maps}

Eight distinct flow regimes have been observed in the current study. These have been identified using the same classification system as used in Morgan, et al. (2012). Instantaneous images of each of the flow regimes are presented in Figure 5. Once again, the flows can be grouped into four more general flow regimes, namely: (1) stratified flow; (2) mixed flow, which is characterised by two distinct continuous phase regions with droplets in each; (3) two-layer flow, which comprised of a dispersed region and a 
continuous, unmixed region, and; (4) dispersed flows. These are the same as those identified in Morgan, et al. (2012); the generalised grouping is presented in Table 2.

A flow regime map relating the flow classifications to the input oil fraction $\phi_{\text {in }}$ and the superficial mixture velocity $U_{\mathrm{m}}$, and based on images such as those in Figure 5, is presented in Figure 6. From this flow regime map it can be seen that the stratified flow regime is observed up to a superficial mixture velocity of $U_{\mathrm{m}}=0.34 \mathrm{~m} \cdot \mathrm{s}^{-1}$; this being $0.27 \mathrm{~m} \cdot \mathrm{s}^{-1}$ higher than the highest superficial mixture velocity (i.e., $U_{\mathrm{m}}=0.07 \mathrm{~m} \cdot \mathrm{s}^{-1}$ ) at which the stratified regime was observed for the square cross-section visualisation section (see Morgan, et al. (2012)). However, above a superficial mixture velocity of $U_{\mathrm{m}}=0.17 \mathrm{~m} \cdot \mathrm{s}^{-1}$ stratified flow is only observed at low oil input phase fractions $\phi_{\text {in }}<0.4$.

Above a superficial mixture velocity of $U_{\mathrm{m}}=0.34 \mathrm{~m} \cdot \mathrm{s}^{-1}$ droplets are found at all oil input phase fractions and the flow is different forms of stratified flow with droplets, i.e., above $U_{\mathrm{m}}=0.34 \mathrm{~m} \cdot \mathrm{s}^{-1}$ the flow has transitioned to dual continuous flow for all oil input phase fractions. Examples of dual continuous flow can be seen in Figure 5(c) and (d). As the superficial mixture velocity increases, the range of oil input phase fractions covered by dual continuous flow diminishes and dispersed flows begin to form at the oil input phase fraction extremes, i.e., very low (near zero) and very high (near unity) oil input phase fractions.

At a superficial mixture velocity of $U_{\mathrm{m}}=0.84 \mathrm{~m} \cdot \mathrm{s}^{-1}$ the flow changes to three-layer flow, see Figure 5(e), at an oil input phase fraction of $\phi_{\text {in }}=0.5$ and to dispersed flows, see Figure 5(f) to (h), at higher oil input phase fractions. Oil dispersions begin to form at an oil input phase fraction of $\phi_{\text {in }}=0.1$ and glycerol/water solution dispersions begin to form at an oil input phase fraction of $\phi_{\text {in }}=0.9$. Comparing Figure 6 with the flow regime map for a square cross-section channel (presented by Morgan, et al., 2012) it is seen that the flow regime transitions occur at higher superficial mixture velocities for the circular tube case. For all the oil input phase fractions investigated, dual continuous flow was not seen for superficial mixture velocity below $U_{\mathrm{m}}=0.34 \mathrm{~m} \cdot \mathrm{s}^{-1}$ whereas; this transition occurred at a superficial mixture velocity of $U_{\mathrm{m}}=0.07 \mathrm{~m} \cdot \mathrm{s}^{-1}$ in the case of the square cross-section duct. 
Furthermore, dispersions were observed at a superficial mixture velocity of $U_{\mathrm{m}} \approx 0.6 \mathrm{~m} \cdot \mathrm{s}^{-1}$ when using the square cross-section duct, whereas the transition to dispersions is first seen at a superficial mixture velocity of $U_{\mathrm{m}} \approx 0.8 \mathrm{~m} \cdot \mathrm{s}^{-1}$ in the circular cross-section duct.

As was the case with the PLIF study presented in Morgan, et al. (2012), the flow regimes are in good general agreement with those from previous studies (Soleimani, 1999; Lovick and Angeli, 2004; Hussain, 2004), yet some flow regimes identified by previous researchers were not observed in the present study. Neither, oil-slugs-in-water (Charles, et al., 1961; Hasson, et al., 1970) nor annular flows (Russell, et al., 1959; Charles, et al., 1961; Hasson, et al., 1970; and, Arirachakaran, et al., 1989) were observed in the current study. This absence of annular flows is consistent with the observations of Angeli (1995), Nädler and Mewes (1995), Soleimani (1999) and Hussain (2004). The absence of annular flows might be attributed to the fact that the oil phase was not dense and viscous enough to sustain an oil core.

The regime transitions observed with the circular pipe section relate much more closely to the transitions observed by Soleimani (1999) than did the results for a square cross-section duct (Morgan, et al., 2012). Three-layer flow was identified in the present campaign, however its onset was not seen until much higher superficial mixture velocities when compared with the flow regime map of the previous study, i.e., at $U_{\mathrm{m}} \approx 0.8 \mathrm{~m} \cdot \mathrm{s}^{-1}$ for the circular duct compared to $U_{\mathrm{m}} \approx 0.4 \mathrm{~m} \cdot \mathrm{s}^{-1}$ for the square duct. The present circular duct data align much more closely with the transition to three layer flow observed by Soleimani (1999) for $U_{\mathrm{m}} \approx 1 \mathrm{~m} \cdot \mathrm{s}^{-1}$, and Hussain (2004) for $U_{\mathrm{m}} \approx 0.8 \mathrm{~m} \cdot \mathrm{s}^{-1}$. Hussain (2004) reported stratified-wavy flow with droplets at superficial mixture velocities up to $U_{\mathrm{m}} \approx 3 \mathrm{~m} \cdot \mathrm{s}^{-1}$; this is considerably higher than the limit found in the current study, in which stratified-wavy flow with droplets was only observed up to mixture velocities of $U_{\mathrm{m}} \approx 0.67 \mathrm{~m} \cdot \mathrm{s}^{-1}$. However, the current value more closely relates to the limit reported by Soleimani (1999), which was a mixture velocity of $U_{\mathrm{m}} \approx 0.7 \mathrm{~m} \cdot \mathrm{s}^{-1}$.

\subsection{Vertical Phase Distribution Profiles}

Figure 7 shows vertical phase profiles $\bar{\phi}(y)$ for superficial mixture velocities $U_{\mathrm{m}}$ in the range 0.11 to $0.42 \mathrm{~m} \cdot \mathrm{s}^{-1}$ at selected input oil fractions $\phi_{\text {in }}$ of: (a) 0.25 , (b) 0.50 and (c) 0.75 , and (d) shows the height of 
the interface (or, mixed) zone as a function of superficial mixture velocities $U_{\mathrm{m}}$ for constant input oil fraction $\phi_{\text {in. }}$ Figure 7 shows similar vertical distribution characteristics to those observed in the experimental study for square cross-section ducts (Morgan, et al., 2012). Specifically, the flow has three distinct regimes: (i) an oil region at the top of the pipe; (ii) a glycerol/water solution region at the bottom of the pipe, and; (iii) a mixed region separating them.

For stratified flows, such as for the $U_{\mathrm{m}}=0.22 \mathrm{~m} \cdot \mathrm{s}^{-1}$ and $\phi_{\text {in }}=0.75$ case presented in Figure $7(\mathrm{c})$, the mixed region covers a narrow vertical band. As the oil input fraction increases for a given superficial mixture velocity, two observations are made: (i) the height of the aqueous glycerol solution at the bottom of the pipe decreases, and; (ii) the vertical range covered by the mixed region increases, as the gradient of the transition from the glycerol/water solution region to the oil region increases. From inspection of Figure 7 (a) to (c) it is seen that the gradient of the transition from the glycerol/water solution region to the oil region (i.e., the height of the mixed region) also increases as the superficial mixture velocity increases for a given oil input fraction. These findings concur with the findings for a square cross-section duct by Morgan, et al. (2012).

From Figure 7(d) it can be seen that as the height of the mixed zone increases with superficial mixture velocity for a given oil input phase fraction. As the superficial mixture velocity is increased, it is seen that the height of the mixed zone increases monotonically with oil input phase fraction. The rate of the increase in the height of the mixed zone with oil input phase fraction is higher at higher superficial mixture velocities. These effects may reflect the tendency of higher turbulence levels (at the higher superficial mixture velocities) to promote mixing of the two initially separated flows.

\subsection{In-Situ Phase Fraction}

This section presents the results for in-situ oil phase fraction $\langle\phi\rangle_{y, t}$. The in-situ oil phase fractions have been calculated using the phase distribution profiles coupled with a numerical integration technique to account for the curvature of the visualisation section wall. For a given image, the instantaneous phase 
fraction $\langle\phi\rangle_{y}(t)$ is calculated via the integral in Eq. (3). For a given run (i.e., a fixed combination of $U_{\mathrm{m}}$ and $\left.\phi_{\text {in }}\right)$ the average in-situ oil fraction $\langle\phi\rangle_{y, t}$ is calculated using Eq. (4).

Figure 8 shows that the in-situ oil fraction $\langle\phi\rangle_{y, t}$ is lower than the input oil fraction $\phi_{\text {in }}$ for almost all flow conditions (as was the case for the square cross-section duct; see Figure 11 in Morgan, et al. (2012)). For a completely mixed flow (i.e., a homogeneous flow) the two phases travel at the same velocity, such that the slip ratio $S$, which is the ratio of the velocity of the lighter phase to that of the heavier phase, is unity. The homogeneous model is represented by the line $S=1$ in Figure 8 . Clearly, from Figure 8 , the flows cannot be described in terms of the homogeneous model, and therefore, two alternative models are investigated in the present paper, namely a laminar drag model and a differential momentum balance model. The results from these models being presented in Figure 8 as $\phi_{\mathrm{mod}, 1}$ and $\phi_{\mathrm{mod}, 2}$, respectively.

The "laminar drag model" for prediction of the in-situ phase fraction was developed by equating the frictional pressure drop in a two layer flow, i.e., by considering an equilibrium between viscous drag due to laminar flow and pressure drop in the pipe. This model is denoted by $\phi_{\text {mod, } 1}$ in Figure 8 . It has been derived using the same basis as $\phi_{\text {mod,lb }}$ in Morgan, et al. (2012). A full derivation is provided in Appendix 1 of Morgan, et al. (2012); the following details and the modifications necessary to account for the new test section geometry (i.e., a circular cross-section opposed to a square cross-section). Firstly, the dimensionless pressure drop is related to the Reynolds number through a Fanning friction factor $f$,

$$
f_{i}=\frac{\Delta p}{\frac{1}{2} \rho_{i} U_{i}^{2}} \frac{D_{i}}{4 L}=\frac{16}{R e_{i}}
$$

Once written for each phase ' $i$ ' and after equating pressure drops $\Delta p / L$, Eq. (9) reduces to,

$$
\frac{\mu_{\mathrm{oil}}}{\mu_{\mathrm{gs}}} \frac{\phi_{\mathrm{in}}}{1-\phi_{\text {in }}} F=1
$$

where, 


$$
F \equiv f(\theta)=\frac{\theta-\sin \theta}{2 \pi-\sin \theta}\left(\frac{2 \pi-\theta}{\theta}\right)^{2}
$$

and,

$$
\frac{Q_{\mathrm{oil}}}{Q_{\mathrm{gs}}}=\frac{\phi_{\mathrm{in}}}{1-\phi_{\mathrm{in}}} ; \operatorname{Re}_{i}=\frac{\delta_{i} U_{i} D_{i}}{\mu_{i}} ; D_{i}=\frac{4 A_{i}}{P_{i}}
$$

Here, $P_{i}$ is defined as the periphery of each layer in contact with the wall; the parameters used to construct Eqs. (9) to (12) are defined in Figure 9.

Finally, the modelled in-situ oil phase fraction $\langle\phi\rangle_{y, t}$ presented in Figure 8 is defined as,

$$
\phi_{\mathrm{mod}, 1}=\langle\phi\rangle_{y, t}=\frac{A_{\mathrm{oil}}}{A_{\mathrm{oil}}+A_{\mathrm{gs}}}=1-\frac{\theta-\sin \theta}{2 \pi}
$$

In Eqs. (11) and (13), $\theta$ is in radians. It should be noted that the contact area between the fluids has not been included in the calculation of $\phi_{\bmod , 1}$.

There is very good agreement between $\phi_{\mathrm{mod}, 1}$ and the experimental results, particularly at lower input oil fractions (typically, $0.1<\phi_{\text {in }}<0.5$ ) and lower superficial mixture velocities.

The second means of comparison, denoted by $\phi_{\mathrm{mod}, 2}$ in Figure 8 , has been derived from a differential momentum balance (Bird, et al., 2001) and is applicable to the special case in which the in-situ oil fraction $\langle\phi\rangle_{y, t}=0.5$ and when the interface level $H$ is at the midpoint of the pipe, i.e., $Y_{\mathrm{oil}}=Y_{\mathrm{gs}}=H=H_{\mathrm{T}} / 2$ in Figure 8. This form of prediction is also in excellent agreement with the experimental results. Therefore, the same conclusions can be drawn as were made for the study using a square cross-section visualisation section (Morgan, et al., 2012). Specifically, that the interface level is adjusting in order to satisfy the increased viscous drag caused by the higher viscosity of the aqueous glycerol solution. 
The input oil fraction $\phi_{\text {in }}$ that results in this value of in-situ oil fraction (i.e., $\langle\phi\rangle_{y, t}=0.5$ ) is given by,

$$
\phi_{\bmod , 2}=\phi_{\text {in }}=\frac{y_{2}}{y_{2}+1}
$$

where $y_{2}=Q_{\mathrm{oil}} / Q_{\mathrm{gs}}$. Now, bearing in mind that $Y_{\mathrm{oil}}=Y_{\mathrm{gs}}=H_{\mathrm{T}} / 2$ we obtain,

$$
y_{2}=\frac{Q_{\mathrm{oil}}}{Q_{\mathrm{gs}}}=\frac{\left\langle u_{x}^{\mathrm{oil}}\right\rangle}{\left\langle u_{x}^{\mathrm{gs}}\right\rangle}
$$

Hence, $\phi_{\text {mod,2 }}=\phi_{\text {in }}$ can be evaluated from the ratio of the average/bulk velocities of each liquid in the cocurrent flow, which is derived from differential momentum balances:

$$
\begin{aligned}
& \left\langle u_{x}^{\mathrm{oil}}\right\rangle=\frac{2}{H_{\mathrm{T}}} \int_{0}^{H_{\mathrm{T}} / 2} u_{x}^{\mathrm{oil}} d y=\frac{\Delta p H_{\mathrm{T}}^{2}}{48 \mu_{\mathrm{oil}} L}\left(\frac{7 \mu_{\mathrm{oil}}+\mu_{\mathrm{gs}}}{\mu_{\mathrm{gs}}+\mu_{\mathrm{oil}}}\right), \\
& \left\langle u_{x}^{\mathrm{gs}}\right\rangle=\frac{2}{H_{\mathrm{T}}} \int_{-H_{\mathrm{T}} / 2}^{0} u_{x}^{\mathrm{gs}} d y=\frac{\Delta p H_{\mathrm{T}}^{2}}{48 \mu_{\mathrm{gs}} L}\left(\frac{7 \mu_{\mathrm{gs}}+\mu_{\mathrm{oil}}}{\mu_{\mathrm{gs}}+\mu_{\mathrm{oil}}}\right) .
\end{aligned}
$$

After substituting Eq. (16) and Eq. (17) into Eq. (15) we obtain,

$$
y_{2}=\frac{\left\langle u_{x}^{\mathrm{oil}}\right\rangle}{\left\langle u_{x}^{\mathrm{gs}}\right\rangle}=\frac{\mu_{\mathrm{gs}}}{\mu_{\mathrm{oil}}}\left(\frac{7 \mu_{\mathrm{oil}}+\mu_{\mathrm{gs}}}{7 \mu_{\mathrm{gs}}+\mu_{\mathrm{oil}}}\right) \text {, }
$$

and finally from Eq. (14):

$$
\phi_{\mathrm{mod}, 2}=\frac{m+7}{m+14+m^{-1}},
$$

where $m=\mu_{\mathrm{gs}} / \mu_{\mathrm{oil}}$ is the ratio of the dynamic viscosities of the two fluids. 
In the homogenous model of two-phase flow the central tenet and key assumption is that the slip ratio $S$, i.e., the velocity ratio of the fluids (defined in Eq. (20)) is unity. In what follows an assessment is made of the suitability of a homogenous flow model approach, based on the slip ratio criterion, compared with separated flow models which account for the existence of slip between the phases,

$$
S=\frac{u_{\mathrm{oil}}}{u_{\mathrm{gs}}}
$$

where $u_{\mathrm{oil}}$ and $u_{\mathrm{gs}}$ are the average velocities of the oil and aqueous glycerol solution. This can be utilised to obtain an expression for the in-situ oil phase fraction $\langle\phi\rangle_{y, t}$ as a function of the input oil fraction $\phi_{\text {in }}$,

$$
S=\frac{u_{\mathrm{oil}}}{u_{\mathrm{gs}}}=\left(\frac{Q_{\mathrm{oil}}}{A_{\mathrm{oil}}}\right) /\left(\frac{Q_{\mathrm{gs}}}{A_{\mathrm{gs}}}\right)=\left(\frac{U_{\mathrm{oil}}}{\langle\phi\rangle_{y, t}}\right) /\left(\frac{U_{\mathrm{gs}}}{1-\langle\phi\rangle_{y, t}}\right)=\frac{U_{\mathrm{oil}}}{U_{\mathrm{gs}}} \frac{1-\langle\phi\rangle_{y, t}}{\langle\phi\rangle_{y, t}},
$$

where,

$$
\frac{U_{\mathrm{oil}}}{U_{\mathrm{gs}}}=\left(\frac{Q_{\mathrm{oil}}}{A}\right) /\left(\frac{Q_{\mathrm{gs}}}{A}\right)=\frac{Q_{\mathrm{oil}}}{Q_{\mathrm{gs}}}=\frac{Q_{\mathrm{oil}} / Q_{\mathrm{T}}}{\left(1-Q_{\mathrm{oil}}\right) / Q_{\mathrm{T}}}=\frac{\phi_{\mathrm{in}}}{1-\phi_{\mathrm{in}}}
$$

Hence, the following expression is obtained for the slip ratio,

$$
S=\frac{\phi_{\text {in }}}{1-\phi_{\text {in }}} \frac{1-\langle\phi\rangle_{y, t}}{\langle\phi\rangle_{y, t}}
$$

One can see that under homogenous flow conditions, i.e., when $S=1$, Eq. (23) reduces to $\phi_{i n}=\langle\phi\rangle_{y, t}$. The homogenous flow model is denoted by the thin sold line labelled $S=1$ in Figure 8 .

It is seen that this approach over-predicts the in-situ phase fraction, with the extent of the over-prediction increasing with an increasing oil input fraction (this will be accounted for and explained to a large extent 
for in the further analysis below). Hence, a slip ratio of $S=1$ does not adequately characterise the experimental results contained herein.

Figure 10 examines the effect of the input oil phase fraction $\phi_{\text {in }}$ on the slip ratio $S$ at constant superficial mixture velocities $U_{\mathrm{m}}$ and includes a comparison with experimental data acquired by Lovick and Angeli (2004) for an oil-water system. From Figure 10 it is seen that for a given oil input phase fraction, as the superficial mixture velocity increases the slip ratio decreases. It can be deduced that as the superficial mixture velocity increases beyond a critical point, the flow is sufficiently well mixed (i.e., dispersed) that it can be adequately described via the homogenous flow model.

Finally, Figure 11 compares the in-situ phase fraction results with the experimental results of Lovick and Angeli (2004) and Russell, et al. (1959) and with the predictions from the laminar drag model $\phi_{\bmod , 1}$ (Eq. (13)). The same conclusions can be drawn from Figure 11 as from the square cross-section duct results of Morgan, et al. (2012); namely that at low superficial mixture velocities the laminar drag model provides excellent agreement with the experimental results. However, as the superficial mixture velocity increases, mixing in the flow increases leading to the entrainment of one liquid as drops into a continuum of the other; when such entrainment occurs, the laminar drag model breaks down. However, for sufficiently high superficial mixture velocities (see the data of Lovick and Angeli (2004) for $U_{\mathrm{m}}=3.00 \mathrm{~m} \cdot \mathrm{s}^{-1}$ ) the flow becomes sufficiently well mixed (i.e., it is in the dispersed flow regime) that it can be adequately characterised by the homogeneous flow model, i.e., $S=1$, hence $\langle\phi\rangle_{y, t}=\phi_{\text {in }}$.

\subsection{Interface Level}

Figure 12 presents the results for the mean $\mu_{H}$ and standard deviation $\sigma_{H}$ of the interface level $H$ as a function of input oil fraction $\phi_{\text {in }}$ for fixed superficial mixture velocities of: (a) $U_{\mathrm{m}}=0.11 \mathrm{~m} \cdot \mathrm{s}^{-1}$ and (b) $0.28 \mathrm{~m} \cdot \mathrm{s}^{-1}$. The results reveal that, for a given superficial mixture velocity $U_{\mathrm{m}}$, the interface level $H$ generally decreases on average as the oil input fraction $\phi_{\text {in }}$ increases. This trend is seen to be more prominent for higher superficial mixture velocities. Furthermore, at the lower superficial mixture velocity 
case, $U_{\mathrm{m}}=0.11 \mathrm{~m} \cdot \mathrm{s}^{-1}$, the interfacial disturbances (characterised by the standard deviation $\sigma_{H}$ ) seem to be enhanced at the higher $\phi_{\text {in }}$; however, this trend cannot be seen at the higher mixture velocity case.

Figure 13 shows the interface level $H$ as a function of oil input phase fraction $\phi_{\text {in }}$ and shows the predicted results for the modified form of the laminar drag model (Eq. (13)), denoted by $H_{\text {mod, } 1}$ and the predictions from the stratified models presented by Hall and Hewitt (1993). The results reveal that, for a given superficial mixture velocity $U_{\mathrm{m}}$, the interface level decreases as the oil input fraction increases. This trend is seen to be more prominent for higher superficial mixture velocities. From comparison of the results for $U_{\mathrm{m}}=0.11$ to $0.28 \mathrm{~m} \cdot \mathrm{s}^{-1}$ it is seen that the rate of decrease in interface level increases as the superficial mixture velocity $U_{\mathrm{m}}$ is increased.

The laminar drag model $H_{\text {mod,1 }}$ predicts the lowering of the interface level $H$ as the oil input fraction $\phi_{\text {in }}$ increases and is in very good agreement with the experimental data points relating to low-velocity stratified flows $\left(U_{\mathrm{m}}=0.11\right.$ to $\left.0.17 \mathrm{~m} \cdot \mathrm{s}^{-1}\right)$ in which the flow contains no droplets. As the laminar drag model does not account for phase break-up and droplet formation the model fails to predict that the interface level decreases for a given oil input phase fraction as the superficial mixture velocity increases.

The Hall and Hewitt (1993) model for liquid-liquid flows generally over-predicts the data. However, the liquids in the present study are such that the oil phase is less dense than the aqueous phase, but also less viscous. This is similar to the case of a gas-liquid flow and the Hall and Hewitt (1993) model for this case is compared with the present data in Figure 13. It is seen that both predictive techniques capture the lowering of the interface level with an increasing oil input phase fraction. However, the liquid-liquid model over-predicts the interface level for all flowing conditions investigated; the difference increases with an increasing oil input phase fraction for a given superficial mixture velocity. The model for gasliquid systems shows excellent agreement with the present experimental data. The breakdown in the ability of the Hall and Hewitt (1993) liquid-liquid model to accurately predict the interface level as the superficial mixture velocity increases can be attributed to the model assuming a flat interface between the two liquids. However, and particularly at higher mixture velocities, the interface is not flat and is covered with waves. The Hall and Hewitt (1993) gas liquid flow model takes account of such waves by assigning an enhanced interfacial friction. 
Figures 14 and 15 present instantaneous images and interface level probability histograms for the points that have been used to construct the mean interface level plots shown in Figure 12. From inspection of the instantaneous images $\left(\mathrm{a}_{1} \rightarrow \mathrm{c}_{1}\right.$ and $\left.\mathrm{x}_{1} \rightarrow \mathrm{z}_{1}\right)$ and the histograms $\left(\mathrm{a}_{2} \rightarrow \mathrm{c}_{2}\right.$ and $\left.\mathrm{x}_{2} \rightarrow \mathrm{z}_{2}\right)$, one can easily see that the mean interface level $\mu_{H}$ decreases with an increasing oil input oil fractions $\phi_{\text {in }}$ for a given superficial mixture velocity $U_{\mathrm{m}}$. From inspection of the images $\left(\mathrm{a}_{1} \rightarrow \mathrm{c}_{1}\right.$ and $\left.\mathrm{x}_{1} \rightarrow \mathrm{z}_{1}\right)$ and the histograms $\left(a_{2} \rightarrow c_{2}\right.$ and $\left.x_{2} \rightarrow z_{2}\right)$, one can conclude that the mean interface level $\mu_{H}$ decreases for an increasing superficial mixture velocity $U_{\mathrm{m}}$ while at the same time the interface level fluctuation range widens. We would expect that at a low superficial mixture velocity $U_{\mathrm{m}}$, when the flow is stratified, that the interface level would be flat and stable $H$ (Shaha, 1999).

The widening of the interface height range with increasing superficial mixture velocity is a manifestation of the corresponding increase of the amplitude of the waves on the interface, and thus to the onset and increase of turbulence in the flow as the superficial mixture velocity increases. Increased turbulence can lead to droplet formation and the entrainment of one phase into a continuum of the other which can further widen the range of interface level heights.

\subsection{Droplet Size Distribution}

Figure 16 shows the effects of: (a) oil input fraction $\phi_{\text {in }}$ and (b) superficial mixture velocity $U_{\mathrm{m}}$, on the mean glycerol/water solution droplet sizes $\mu_{d, \text { gs }}$. Figure 16 presents mean chord lengths, as well as equivalent spherical diameter values that correspond to the direct measured droplet chord lengths, calculated in MATLAB based upon an assumption of a uniform distribution of droplets in the flow and a spherical droplet shape. Figures 17 and 18 present instantaneous images $\left(\mathrm{a}_{1} \rightarrow \mathrm{c}_{1}\right.$ and $\left.\mathrm{x}_{1} \rightarrow \mathrm{z}_{1}\right)$ and probability histograms $\left(\mathrm{a}_{2} \rightarrow \mathrm{c}_{2}\right.$ and $\left.\mathrm{x}_{2} \rightarrow \mathrm{z}_{2}\right)$ for the droplet chord diameter (direct measurement) points labelled $\mathrm{a} \rightarrow \mathrm{c}$ and $\mathrm{x} \rightarrow \mathrm{z}$ in Figure 16.

In particular, Figure 16(a) indicates that for a fixed superficial mixture velocity $U_{\mathrm{m}}$, the average glycerol/water solution droplet diameter $\mu_{d, \mathrm{gs}}$ increases at a constant rate with an increasing oil input fraction up to $\phi_{\text {in }}=0.5$. However, as the oil input fraction $\phi_{\text {in }}$ is increased further, the average 
glycerol/water solution droplet diameter decreases monotonically, i.e., the mean droplet diameter is shown to peak at an oil input fraction of $\phi_{\text {in }}=0.5$ with a value of $\mu_{d, \mathrm{gs}}=4.1 \mathrm{~mm}$, at a superficial mixture velocity of $U_{\mathrm{m}}=0.22 \mathrm{~m} \cdot \mathrm{s}^{-1}$. As was seen with the PLIF study presented in Morgan, et al. (2012), this result is consistent with the fining of $\mathrm{Pal}$ (1993) who observed a peak in the droplet size at the phase inversion point, $\phi_{\text {in,water }}=0.3$. This is also consistent with the findings of Liu (2005) who also observed a maximum in the droplet size at the phase inversion point.

Figure 16(b) shows results for mean glycerol/water solution droplet size as a function of superficial mixture velocity $U_{\mathrm{m}}$ for a given (fixed) input oil fraction of $\phi_{\mathrm{in}}=0.25$. The mean glycerol/water solution droplet diameter $\mu_{d, \mathrm{gs}}$ increases slightly (by about $6 \%$ ) and then decreases (by about 14\%) as a result of the increase in the superficial mixture velocity $U_{\mathrm{m}}$ from $0.22 \mathrm{~m} \cdot \mathrm{s}^{-1}$ to $0.28 \mathrm{~m} \cdot \mathrm{s}^{-1}$, and then to $0.42 \mathrm{~m} \cdot \mathrm{s}^{-1}$.

Figures 17 and 18 show probability histograms of glycerol/water solution droplet size and instantaneous images for the points $\mathrm{a} \rightarrow \mathrm{c}$ and $\mathrm{x} \rightarrow \mathrm{z}$ in Figure 16(a) and (b), respectively. From Figure 17 it can be seen that as the oil input fraction is increased for a given superficial mixture velocity (in this case, $U_{\mathrm{m}}=0.22 \mathrm{~m} \cdot \mathrm{s}^{-1}$ ) the glycerol/water solution droplets occupy the same diameter range though the distribution becomes more uniform with increasing oil input fraction. Figure 18( $\left.\mathrm{x}_{2}\right)$ shows that, at a low superficial mixture velocity of $U_{\mathrm{m}}=0.22 \mathrm{~m} \cdot \mathrm{s}^{-1}$, there is a clear preference for droplets of a certain size. The preference for this size is maintained as the superficial mixture velocity is increased (firstly to $U_{\mathrm{m}}=0.28 \mathrm{~m} \cdot \mathrm{s}^{-1}$ and then on to $U_{\mathrm{m}}=0.42 \mathrm{~m} \cdot \mathrm{s}^{-1}$ ), however, the probability for smaller droplets decreases as the superficial mixture velocity is increased.

Figure 19 presents probability histograms for the point labelled "a" in Figure 16(a), i.e., for a flow with a superficial mixture velocity $U_{\mathrm{m}}=0.22 \mathrm{~m} \cdot \mathrm{s}^{-1}$ and oil input fraction of $\phi_{\mathrm{in}}=0.25$ (these are the same flow conditions for the point labelled " $x$ " in Figure 16(b)). Figure 19(a) presents a probability histogram distribution of the direct measurements of droplet chord length, whereas Figure 19(b) presents the equivalent probability histogram distribution of the associated droplet diameters back-calculated from the chord measurements, and the assumptions of uniform and spherical droplets mentioned above. The histograms in Figure 19 show that, once the droplet chord data is processed to provide droplet diameters, the operation leads to a shift in probability towards larger droplet size values, as expected. 


\subsection{Velocity Profiles}

Figure 20 shows velocity profiles for superficial mixture velocities in the range $U_{\mathrm{m}}=0.11$ to $0.42 \mathrm{~m} \cdot \mathrm{s}^{-1}$ at oil input fractions $\phi_{\text {in }}$ of (a) 0.25 ; (b) 0.50 ; and (c) 0.75 . For a given run (i.e., a fixed superficial mixture velocity $U_{\mathrm{m}}$ and fixed oil input fraction $\phi_{\mathrm{in}}$ ) the velocity profile has been divided by the superficial mixture velocity of that run, i.e., it has been normalised. From inspection of Figure 20 it can be seen that once normalised, the velocity profiles for a given oil input fraction $\phi_{\text {in }}$ collapse to a 'generic' profile that is largely independent of the superficial mixture velocity (though with some exceptions). However, there is a region that retains dependence to the superficial mixture velocity, namely the interface region. This dependence can be attributed to the transition through the different flow regimes that are encountered as the superficial mixture velocity is increased.

Though the velocity of the two phases must be close to being the same at the interface, there may be thin boundary layers in the interface region in either or both phases in which the velocity may change significantly. These changes may manifest themselves as an apparent step change in velocity from one phase to the other as the interface region is traversed. The "step-change" at the interface is seen to be most prominent for the lowest superficial mixture velocities investigated, $U_{\mathrm{m}}=0.11$ to $0.22 \mathrm{~m} \cdot \mathrm{s}^{-1}$. As previously discussed in Section 3.3 (see in particular, Figure 10), the slip ratio $S$ between the two liquid phases is highest for these conditions. Comparing Figure 20(a) and (b), the "step-change" at the interface is seen to increase with oil input phase fraction. Ultimately, if there is sufficient shear (i.e., a sufficient velocity difference) across the interface, this will give rise to an instability which can cause waves at the common interface and the onset of a transition to other flow regimes, first dual continuous flow and ultimately dispersed flow which have their own characteristic velocity profiles.

Figure 21 presents a normalised velocity profile in relation to a corresponding instantaneous image of a stratified flow with the following input conditions: $U_{\mathrm{m}}=0.28 \mathrm{~m} \cdot \mathrm{s}^{-1}$ and $\phi_{\text {in }}=0.38$. Inspection of the velocity profile (a) in relation to the flow image (b) shows that, from the bottom of the test section, the velocity profile follows a parabolic curve up to the interface, characteristic of a velocity profile for laminar flow. The velocity profile in the upper region is more representative of a turbulent flow. 
The results shown in Figure 21 are consistent with the Reynolds numbers of the individual phases which can be calculated using a combination of the volumetric flow-rates $\left(Q_{i}\right)$ of the individual phases coupled with the respective in-situ phase fractions, such that,

$$
R e_{i}=\frac{\rho_{i} U_{i} D_{i}}{\mu_{i}},
$$

where,

$$
U_{i}=\frac{Q_{i}}{\langle\phi\rangle_{y, t}\left(\frac{\pi D_{i}^{2}}{4}\right)},
$$

and,

$$
\langle\phi\rangle_{y, t} A=A_{i}=\frac{\pi D_{i}^{2}}{4}
$$

where $A$ is the total cross-sectional area of the pipe and $A_{i}$ is the cross-sectional area occupied by phase $i$.

Figure 21 indicates that the aqueous glycerol solution is still travelling as laminar flow $\left(R e_{\mathrm{gs}}=55\right)$; this is supported by the shape of the velocity profile for the aqueous glycerol solution in Figure 21(a). However, the Reynolds number for the oil phase is not in the laminar region $\left(R e_{\mathrm{oil}}=3414\right)$. This is in line with the shape of the velocity profile of the oil phase in Figure 21(a), which is steeper closer to the pipe wall and so more characteristic of a turbulent flow. It should be emphasised that the results presented in Figure 21 and the associated calculations of the Reynolds numbers of the individual phases were for runs in which the two phases remained separated and there are no droplets present, i.e., for stratified flow only.

Figure 22 presents a velocity profile and an instantaneous flow image for $U_{\mathrm{m}}=0.84 \mathrm{~m} \cdot \mathrm{s}^{-1}$ and $\phi_{\text {in }}=0.50$. Here the flow regime is three-layer flow. Three distinct regions to the profile are identifiable, these being: (i) a single-phase glycerol/water solution region (i.e., up to $H \approx 10 \mathrm{~mm}$ ); (ii) a highly dispersed 
region (i.e., from $H \approx 10 \mathrm{~mm}$ up to $H \approx 20 \mathrm{~mm}$ ), and; (iii) a single phase oil region up to the top of the channel. From the shape of the velocity profile it is deduced that all three regions are turbulent.

Figures 23 and 24 show velocity profiles and corresponding instantaneous flow images for various flow conditions. In Figure 23 we observe results for: (1) $U_{\mathrm{m}}=0.67 \mathrm{~m} \cdot \mathrm{s}^{-1}$ and $\phi_{\text {in }}=0.75$, and (2) $U_{\mathrm{m}}=0.56 \mathrm{~m} \cdot \mathrm{s}^{-1}$ and $\phi_{\mathrm{in}}=0.75$, respectively. In both cases, there is a thin layer (up to $H \approx 2 \mathrm{~mm}$ ) of glycerol-solution on the pipe wall. Above $H \approx 2 \mathrm{~mm}$ there is a second layer arising from the flow of a glycerol-solution droplet region above the glycerol-solution film, travelling at a different velocity to that of the film. Figure 24 presents a normalised velocity profiles $\left(a_{1} \rightarrow a_{2}\right)$ and instantaneous images $\left(\mathrm{b}_{1} \rightarrow \mathrm{b}_{2}\right)$ for dispersed flows. Figure $24(1)\left(U_{\mathrm{m}}=0.84 \mathrm{~m} \cdot \mathrm{s}^{-1}\right.$ and $\left.\phi_{\text {in }}=0.25\right)$ shows results for a dispersion of oil droplets in the glycerol/water solution phase, and Figure $24(2)\left(U_{\mathrm{m}}=0.83 \mathrm{~m} \cdot \mathrm{s}^{-1}\right.$ and $\left.\phi_{\text {in }}=0.90\right)$ shows results for a dispersion of glycerol/water solution droplets in oil. Inspection of the velocity profile in Figure 24(a1) in relation to the instantaneous flow image shown in Figure 24(b1) shows that the step changes in the velocity profile observed at $H \approx 24 \mathrm{~mm}$ and $H \approx 20 \mathrm{~mm}$ are due to discrete layers of oil droplets flowing at different velocities. 


\section{Conclusions}

The experimental campaign that is the subject of this paper is a development of the square duct section study presented in Morgan, et al. (2012). However, the current investigation makes use of a test section with a geometry more representative of practical/industrial pipeline systems (i.e., with a circular crosssection). In addition, the characterisation of the investigated flows has been developed herein to include both phase information and velocity profiles.

The following main conclusions can be drawn from this work:

(1) A non-intrusive, spatiotemporally resolved optical measurement technique, incorporating planar laser-induced fluorescence (PLIF) simultaneously with particle velocimetry (PIV/PTV), was developed and used to measure co-current liquid-liquid flows in a horizontal circular pipe section. The two liquid phases (Exxsol D80 oil and an aqueous glycerol solution) had matched refractive indices. The borosilicate glass test section wall had a different refractive index and this gave rise to image distortion, which required the development of a method for the successful correction of the raw images and the provision of undistorted images for further analysis.

(2) The images obtained were processed to yield information on flow regime, vertical phase distribution, in-situ phase fraction, interface level, drop size distribution and velocity profile. In these experiments it was not necessary to add seeding particles to the flow, since it was found that a sufficient (though very small) concentration of micro-bubbles and micro-droplets existed naturally in the flow which could be used to track the fluid motion using the PIV/PTV method. The velocity profile was determined by applying a standard PIV/PTV algorithm.

(3) Eight distinct flow regimes have been observed. These been grouped into the following four flow types: (1) stratified flow; (2) mixed flow (i.e., a flow with two distinct continuous phase regions with droplets); (3) continuous oil-phase dispersion, and; (4) continuous aqueous-phase dispersion. Comparing the results to those obtained for a square cross-section (Morgan et al., 2012) it is seen that stratified flows persist to considerably higher superficial mixture velocities. The transition to dispersed flow also occurs at much higher velocities in the circular geometry. 
(4) The data on the interface level were compared with several predictive models. For lower flow velocities, a stratified laminar-laminar model was found to work well, but a more classical (smooth interface) two-fluid model was needed to predict the data as the flow rate increased. At the highest flow rates, it was necessary to use a model (namely that of Hall and Hewitt, 1993) which took account of the waviness of the interface.

(5) The drop sizes observed were of the order of $3-5 \mathrm{~mm}$. Drop size passed through a peak with increasing input oil fraction at a given fluid velocity, and with increasing fluid velocity at a constant oil fraction.

(6) From the bottom of the test section to the interface, the velocity profile followed a parabolic curve typifying a laminar flow. The velocity profile in the upper region was typically more representative of turbulent flow.

(7) A simple stratified laminar-laminar flow drag model (tailored for a circular cross-section pipeline) has been shown to give good agreement with experimental in-situ phase fraction and interface level results. Moreover, the gas-liquid interface level predictive technique presented by Hall and Hewitt (1993) has also been shown to agree with the current experimental results. 


\section{References}

Arirachakaran, S., Oglesby, K. D., Malinowsky, M. S., Shoham, O. and Brill, J. P., 1989. An analysis of oil/water phenomena in horizontal pipes. SPE Paper 18836, SPE Prod. Oper. Symp., Oklahoma City, March 13-14, pp. 155-167.

Angeli, P., 1996. Liquid-liquid dispersed flows in horizontal pipes. Ph.D. thesis, Imperial College London, London.

Angeli, P. and Hewitt, G. F., 2000a. Drop size distributions in horizontal oil-water dispersed flows. Chem. Eng. Sci. 55 (16), 3133-3143.

Angeli, P. and Hewitt, G. F., 2000b. Flow structure in horizontal oil-water flow. Int. J. Multiph. Flow 26 (7), 1117-1140.

Barbosa, J. R., Govan, A. H. and Hewitt, G. F., 2001. Visualisation and modelling studies of churn flow in a vertical pipe. Int. J. Multiph. Flow 27 (12), 2105-2127.

Bird, R. B., Stewart, W. E. and Lightfoot, E. N., 2001. Transport Phenomena. John Wiley and Sons, New York.

Chen, J., 2003. Study on oil-water two phase pipe flow. Ph.D. thesis, University of Petroleum, Beijing.

Charles, M. E., Govier, G. W. and Hodgson, G. W., 1961. The horizontal pipeline flow of equal density oil-water mixtures. Can. J. Chem. Eng. 39 (1), 27-36.

Charles, M. E. and Lilleleht, L. U., 1966. Correlation of pressure gradients for the stratified laminarturbulent pipeline flow of two immiscible liquids. Can. J. Chem. Eng. 44 (1), 47-49.

Coulaloglou, C. A. and Tavlarides, L. L., 1976. Drop size distribution and coalescence frequencies of liquid-liquid dispersions in flow vessels. AIChE J. 22 (2), 289-297.

Du, H., Fuh, R. A., Li, J ., Corkan, A. and Lindsey, J. S., 1998. PhotochemCAD: A computer-aided design and research tool in photochemistry. Photochem. Photobiol. 68 (2), 141-142.

Guzhov, A. I., Grishin, A. D., Medredev, V. F. and Medredeva, O. P., 1973. Emulsion formation during the flow of two liquids in a pipe. Neft. Khoz. 8, 58-61 (in Russian).

Hall, A. R. and Hewitt, G. F., 1993. Application of two-fluid analysis to laminar stratified oil-water flows. Int. J. Multiph. Flow 19 (4), 711-717. 
Hasson, D., Mann, U. and Nir, A., 1970. Annular flow of two immiscible liquids. Can. J. Chem. Eng. 48 (5), 514-520.

Hewitt, G. F., Jayanti, S., and Hope, C. B., 1990. Structure of thin liquid films in gas-liquid horizontal flow. Int. J. Multiph. Flow 16 (6), 951-957.

Hussain, S. A., 2004. Experimental and computational studies of liquid-liquid dispersed flows. Ph.D. thesis, Imperial College London, London.

Ishii, M. and Mishima, K., 1984. Two-fluid model and hydrodynamic constitutive relations. Nucl. Eng. Des. $82(2-3), 107-126$.

Ioannou, K., Nydal, O. J. and Angeli, P., 2005. Phase inversion in dispersed liquid-liquid flows. Exp. Therm. Fluid Sci. 29 (3), 331-339.

Kashid, M. N. and Agar, D. W., 2007. Hydrodynamics of liquid-liquid slug flow capillary microreactor: Flow regimes, slug size and pressure drop. Chem. Eng. J. 131 (1-3), 1-13.

Liu, L., 2005. Optical and computational studies of liquid-liquid flows. Ph.D. thesis, Imperial College London, London.

Lovick, J. and Angeli, P., 2004. Experimental studies on the dual continuous flow pattern in oil-water flows. Int. J. Multiph. Flow 30 (2), 139-157.

Malinowsky, M., 1975. An experimental study of oil-water and air-oil-water flowing mixtures in horizontal pipes. Ph.D. thesis, University of Tulsa, Tulsa.

Markides, C. N. and Mastorakos, E., 2006. Measurements of scalar dissipation in a turbulent plume with planar laser-induced fluorescence of acetone. Chem. Eng. Sci. 61 (9), 2835-2842.

Morgan, R. G., Markides, C. N., Hale, C. P., Hewitt, G.F., 2012. Horizontal liquid-liquid flow characteristics at low superficial velocities using laser-induced fluorescence. In. K. Muliph. Flow 43, 101-117.

Nädler, M. and Mewes, D., 1995. Effects of the liquid viscosity on the phase distributions in horizontal gas-liquid slug flow. Int. J. Multiph. Flow 21 (2), 253-266.

Ng, T. S., 2002. Interfacial structure of stratified pipe flow. Ph.D. thesis, Imperial College London, London.

Pal, R., 1993. Pipeline flow of unstable and surfactant-stabilized emulsions. AIChE J. 39 (11), 17541764. 
Russell, T. W. F. and Charles, M. E., 1959. The effect of the less viscous liquid in the laminar flow of two immiscible liquids. Can. J. Chem. Eng. 37 (1), 18-24.

Russell, T. W. F., Hodgson, G. W. and Govier, G. W., 1959. Horizontal pipeline flow of mixtures of oil and water. Can. J. Chem. Eng. 37 (1), 9-17.

Sajjadi, S., Zerfa, M. and Brooks, B. W., 2002. Dynamic behaviour of drops in oil/water/oil dispersions. Chem. Eng. Sci. 57 (4), 663-675.

Selker, A. H., Sleicher, Jr., C. A., 1965. Factors affecting which phase will disperse when immiscible liquids are stirred together. Can. J. Chem. Eng. 43 (6), 298-301.

Shaha, J., 1999. Phase interactions in transient stratified flow. Ph.D. thesis, Imperial College London, London.

Simmons, M. J. H and Azzopardi, B. J., 2001. Drop size distributions in dispersed liquid-liquid pipe flow. Int. J. Multiph. Flow 27 (5), 843-859.

Soleimani, A., 1999. Phase distribution and associated phenomena in oil-water flows in horizontal tubes. Ph.D. thesis, Imperial College London, London.

Taitel, Y. and Dukler, A. E., 1976. A model for predicting flow regime transitions in horizontal and near horizontal gas-liquid flow. AIChE J. 22 (1), 47-55.

Tobin, T. and Ramkrishna, D., 1992. Coalescence of charged droplets in agitated liquid-liquid dispersions. AIChE J. 38 (8), 1199-1205.

Tokeshi, A., Minagawa, T., Uchiyama, K., Hibara, A., Sato, K., Hisamoto, H. and Kitamori, T., 2002. Continuous-flow chemical processing on a microchip by combining microunit operations and a multiphase flow network. Anal. Chem. 74 (7), 1565-1571.

Ward, J. P. and Knudsen, J. G., 1967. Turbulent flow of unstable liquid-liquid dispersions: drop sizes and velocity distributions. AIChE J. 13 (2), 356-365.

Wegmann, A. and von Rohr, P. R., 2006. Two phase liquid-liquid flows in pipes of small diameters. Int. J. Multiph. Flow 32 (8), 1017-1028.

Yeo, L. Y., Matar, O. K., Perez de Ortiz, E. S. and Hewitt, G. F., 2002. A simple predictive tool for modelling phase inversion in liquid-liquid dispersions. Chem. Eng. Sci. 57 (6), 1069-1072. 


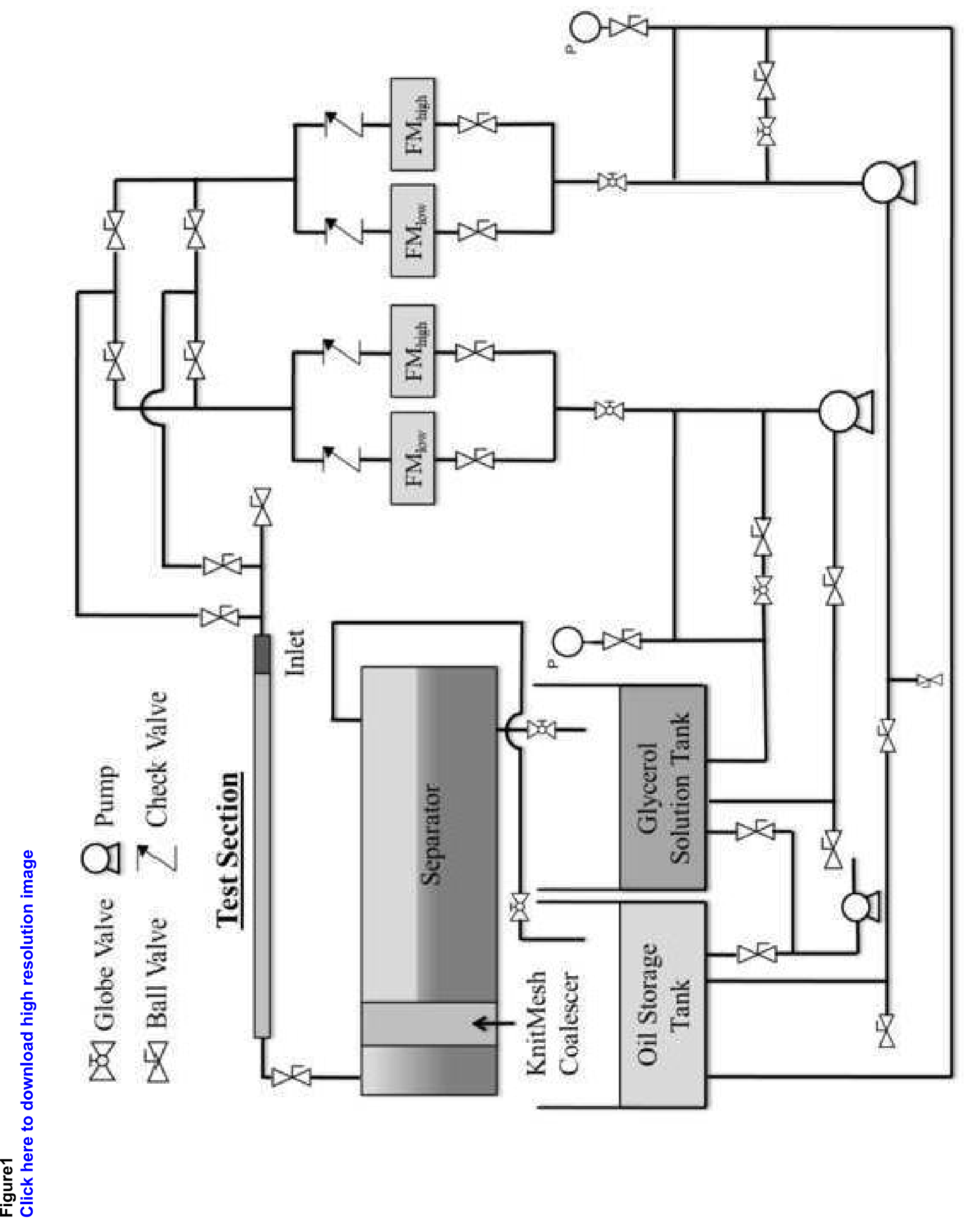



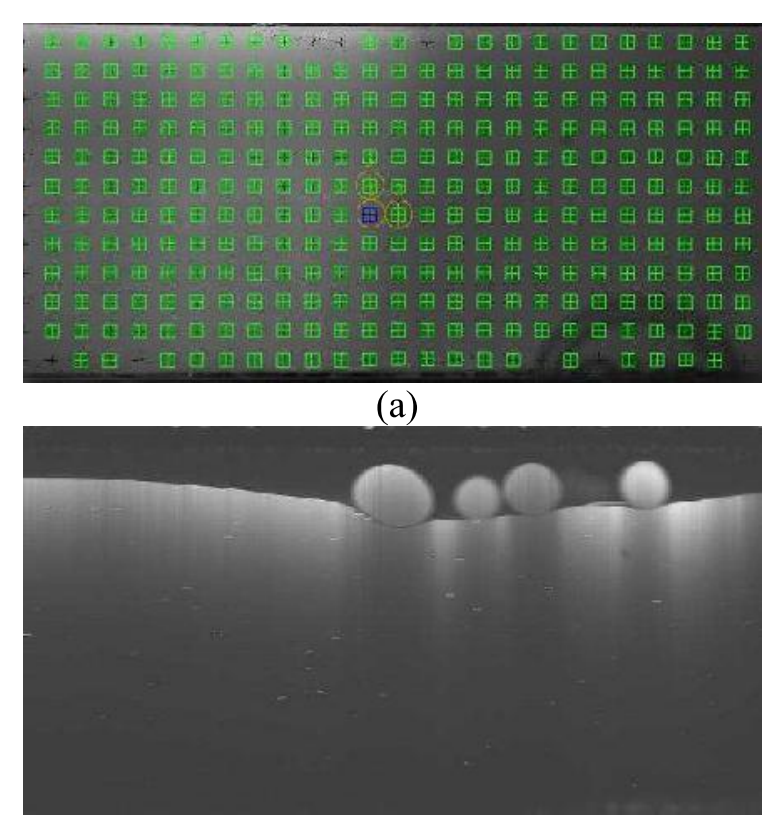

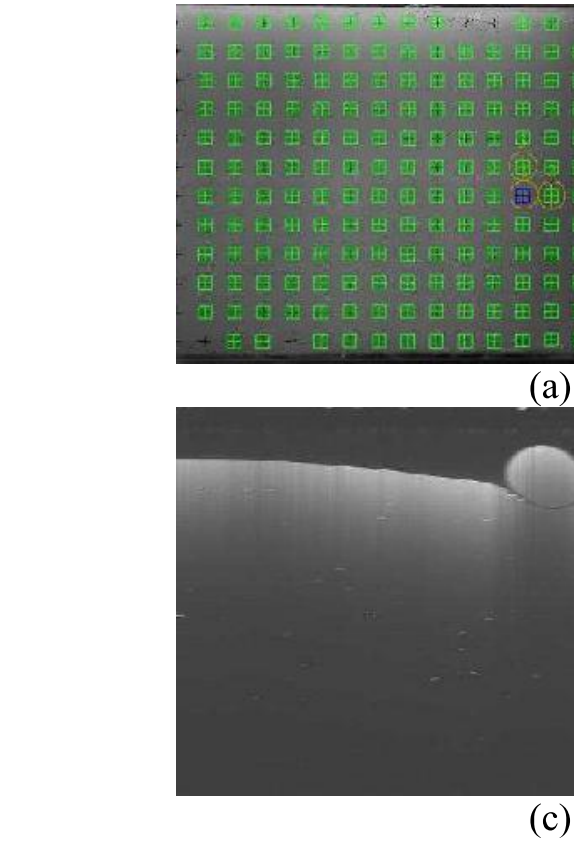

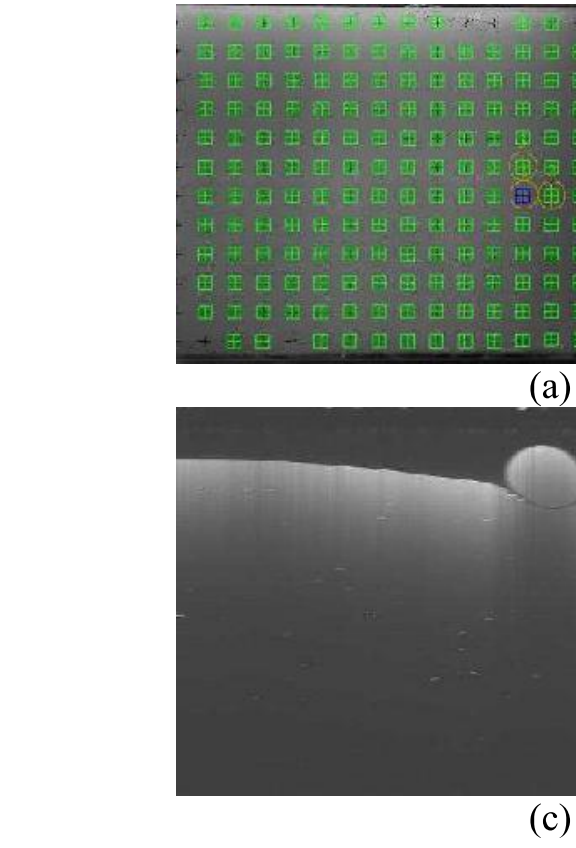

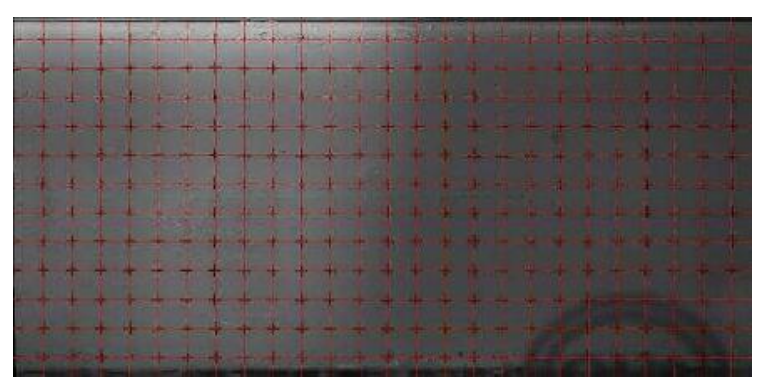

(b)

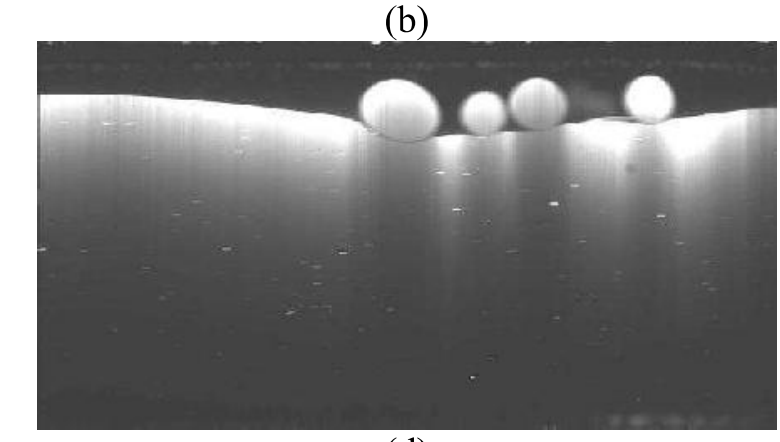

(d)

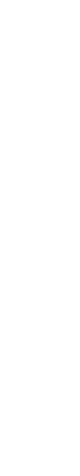




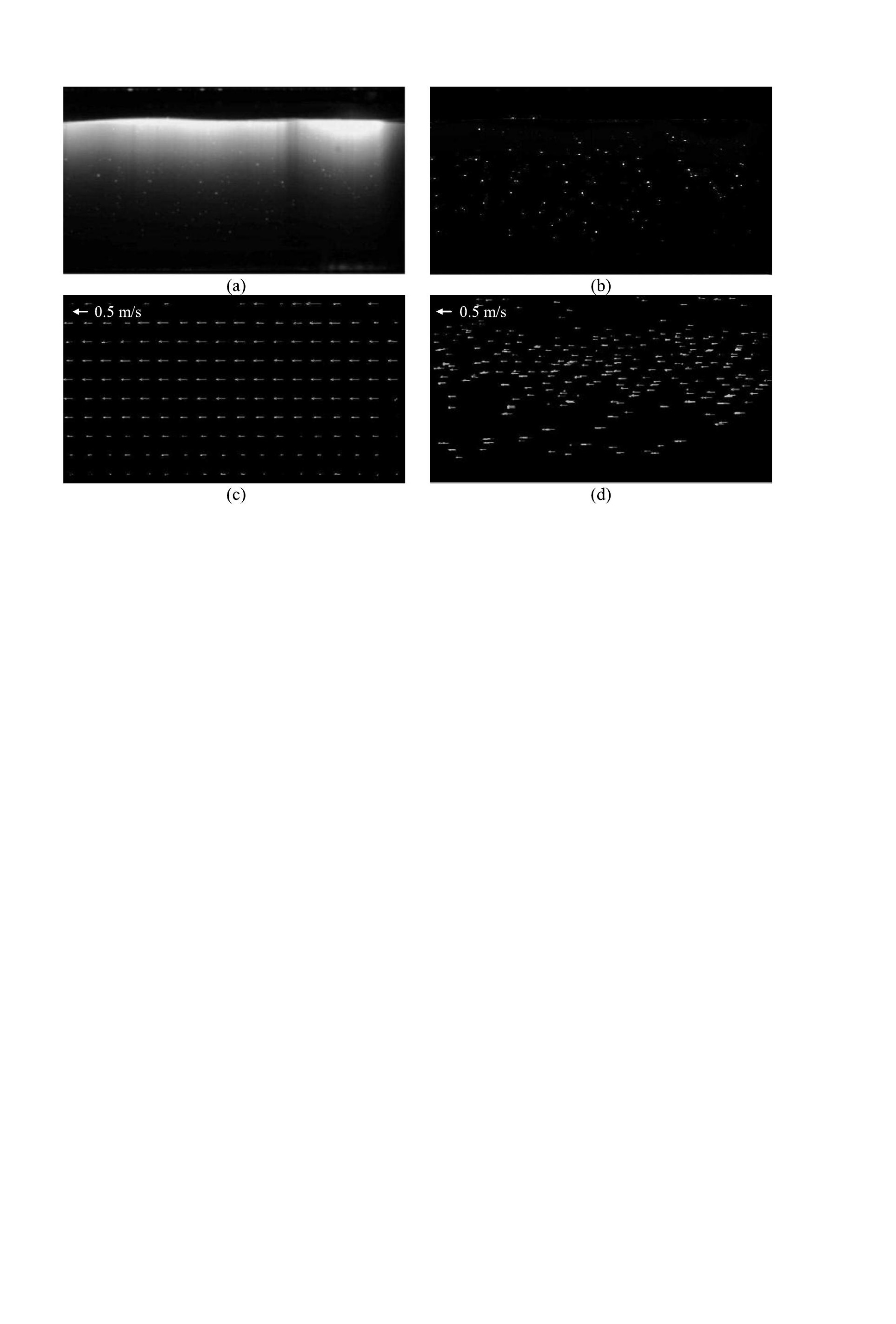

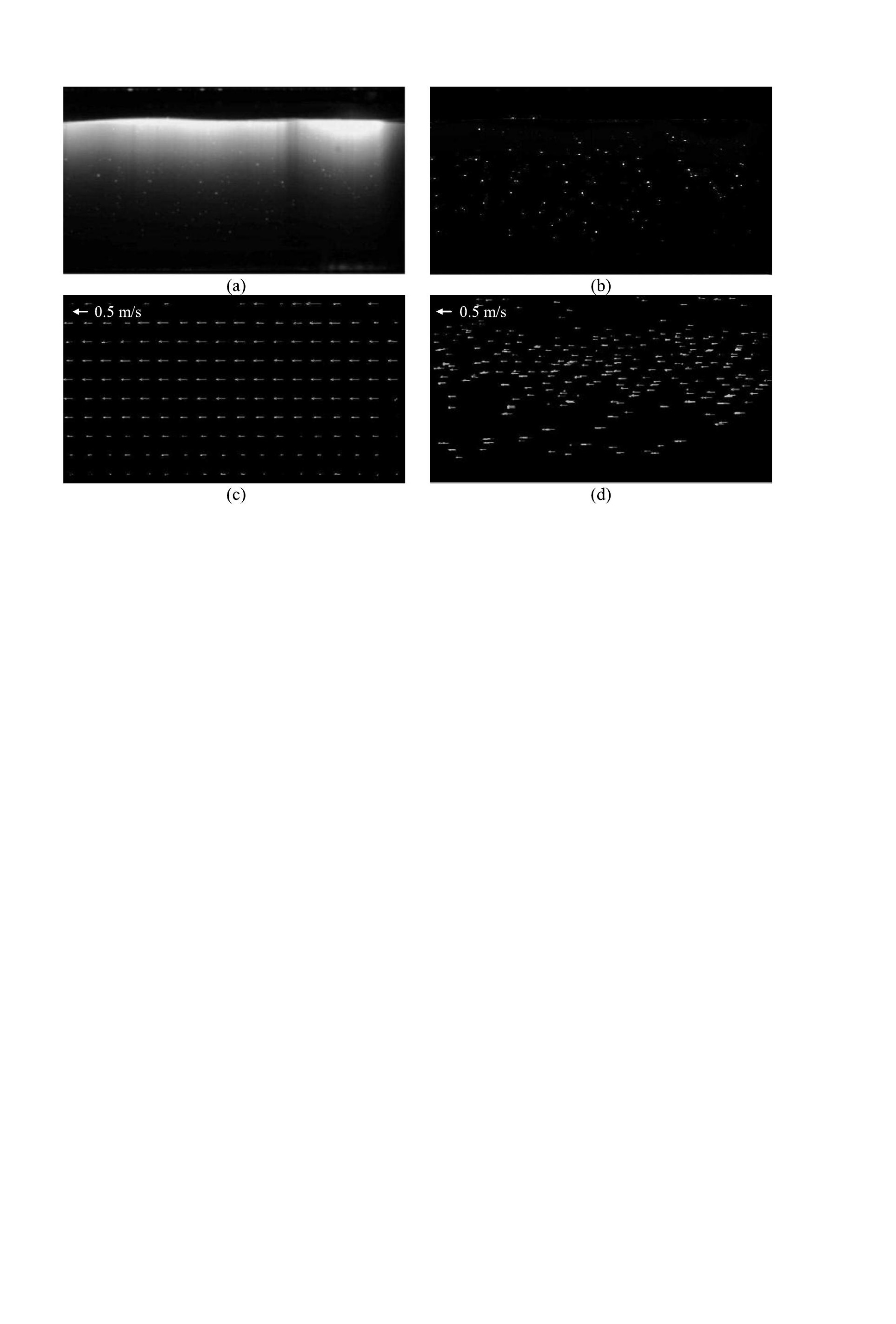

(a)
$60.5 \mathrm{~m} / \mathrm{s} \ldots$
$\ldots \ldots$
$\ldots \ldots$
$\ldots \ldots$
$\ldots \ldots$

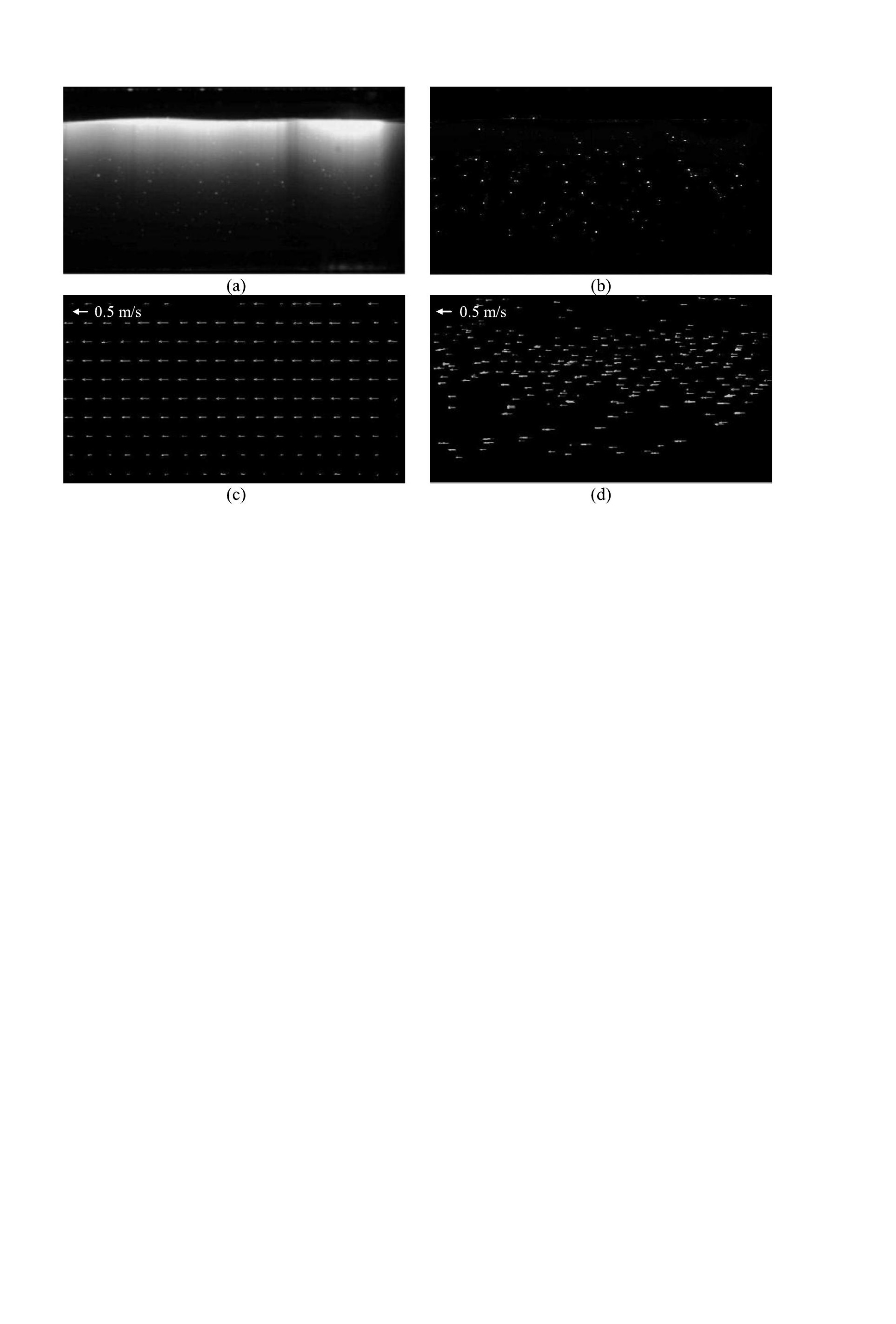

(a)
$60.5 \mathrm{~m} / \mathrm{s} \ldots$
$\ldots \ldots$
$\ldots \ldots$
$\ldots \ldots$
$\ldots \ldots$

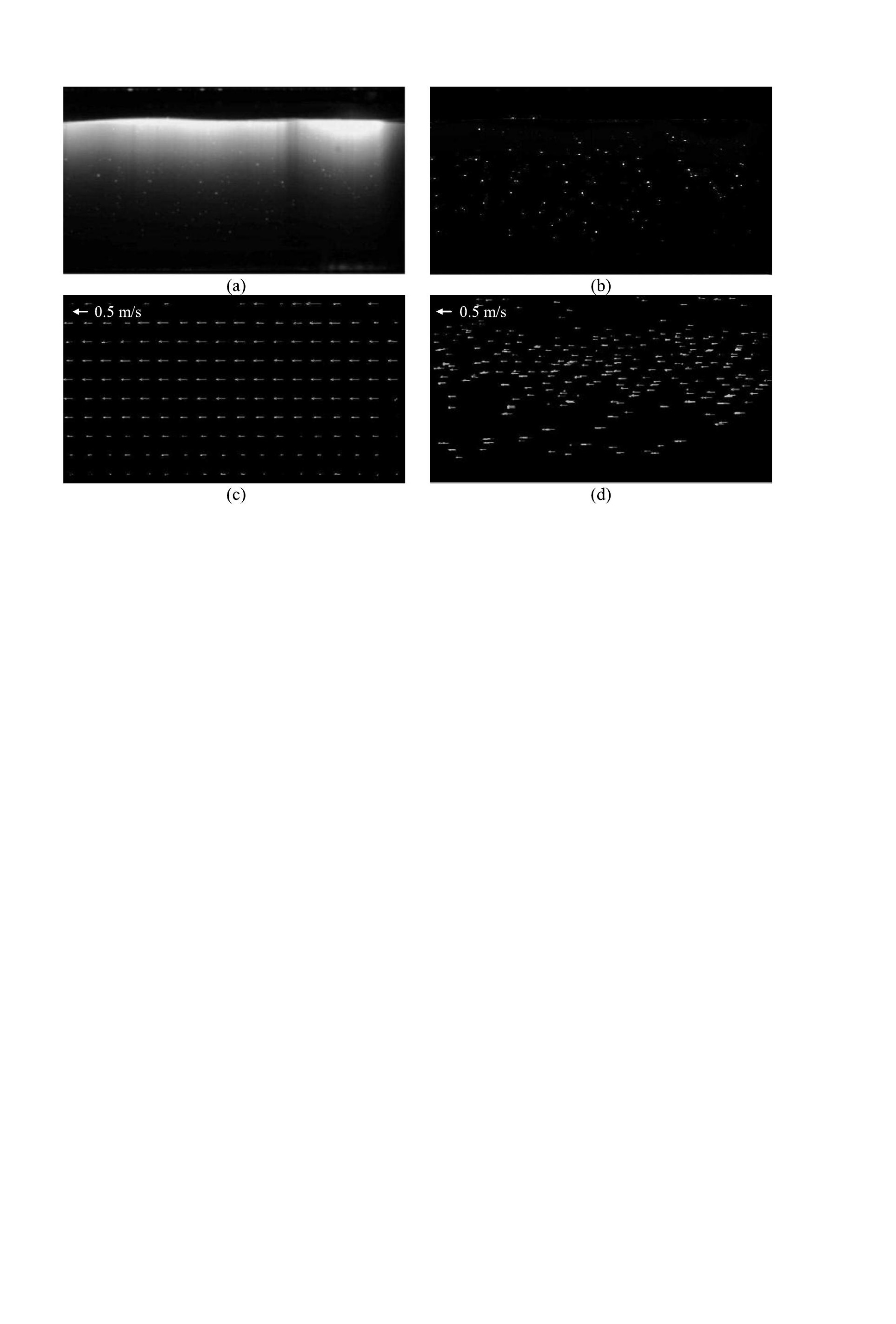

(b)

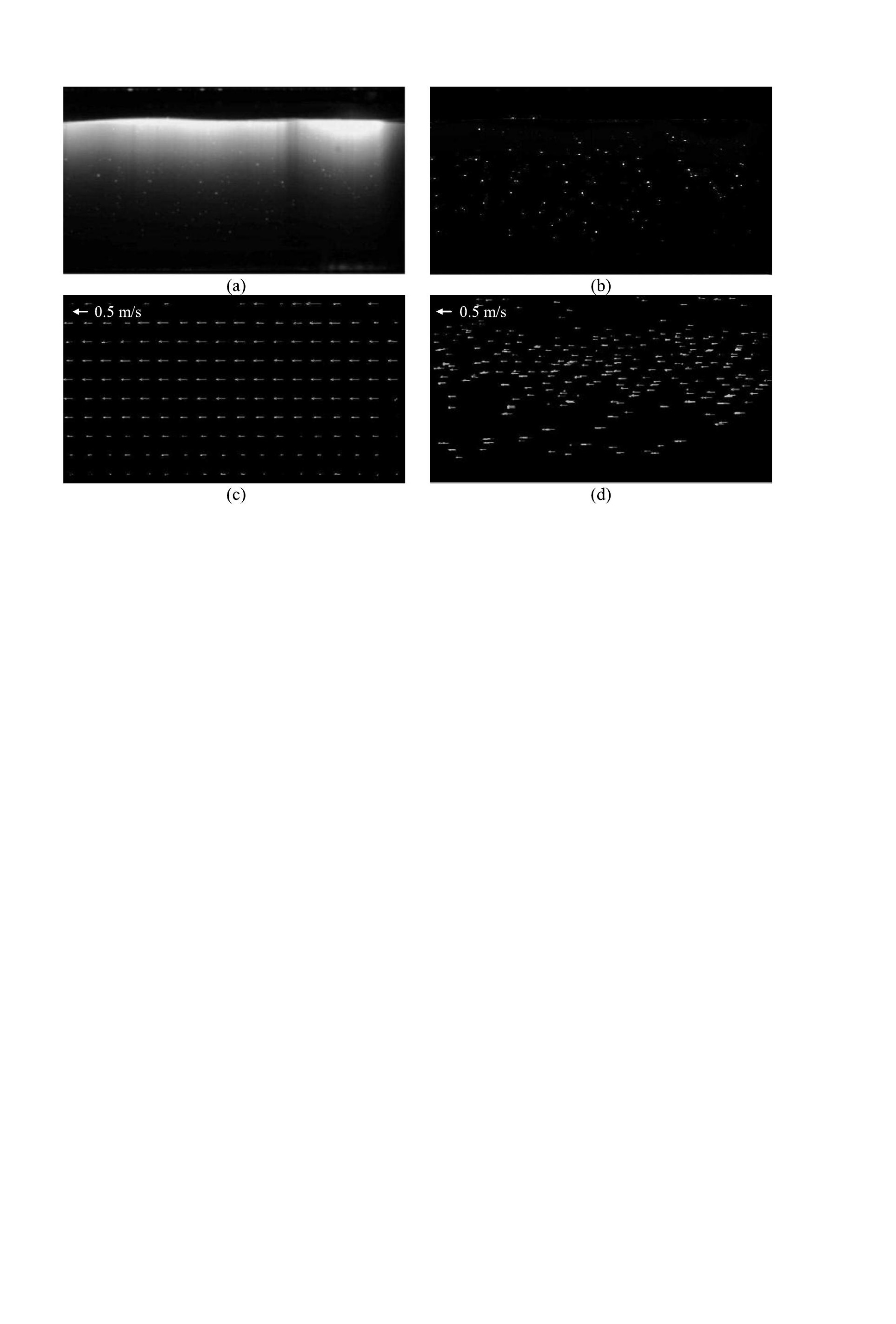

(d)$$
\text { ) }
$$$$
\text { (a) }
$$

政

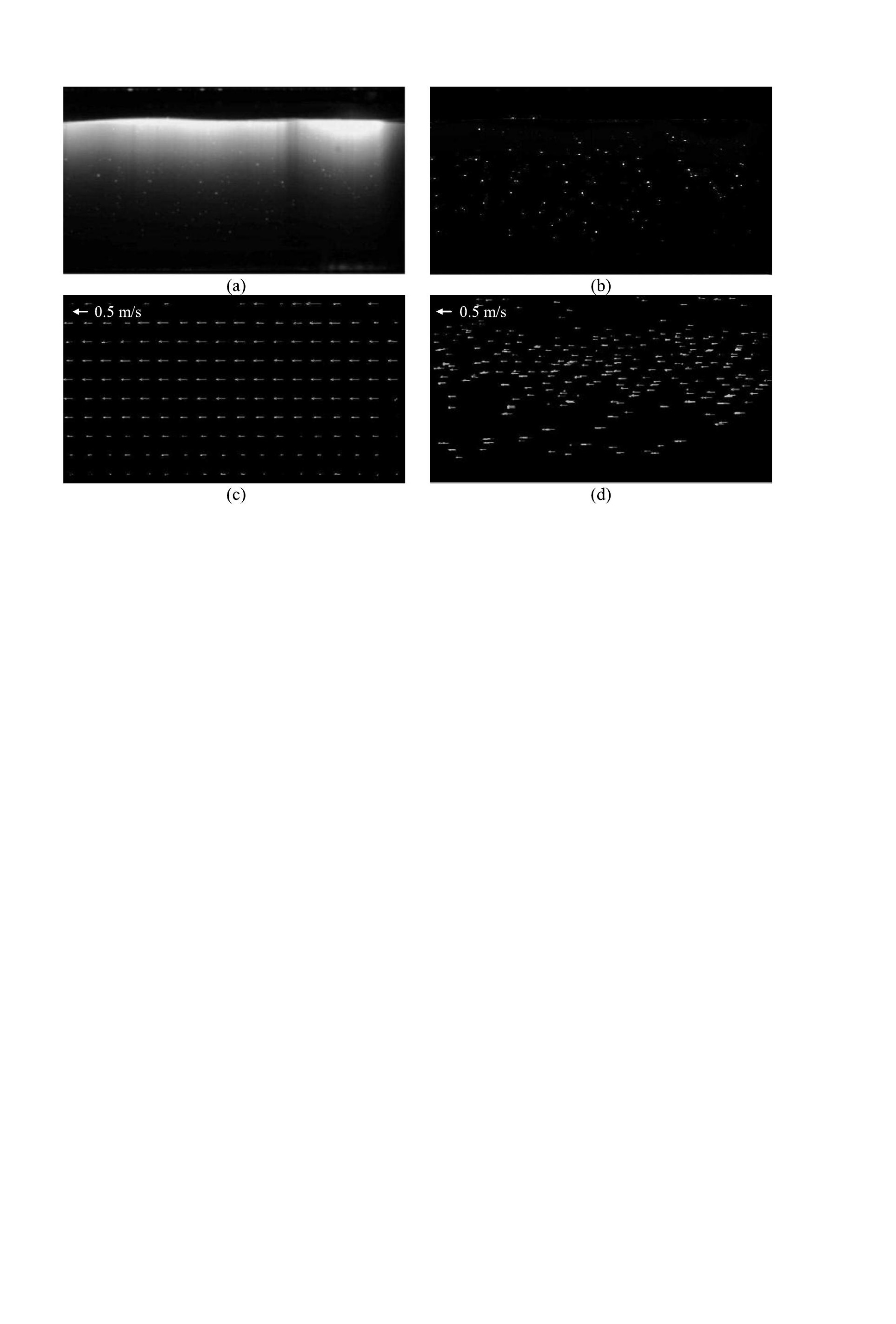

(c)
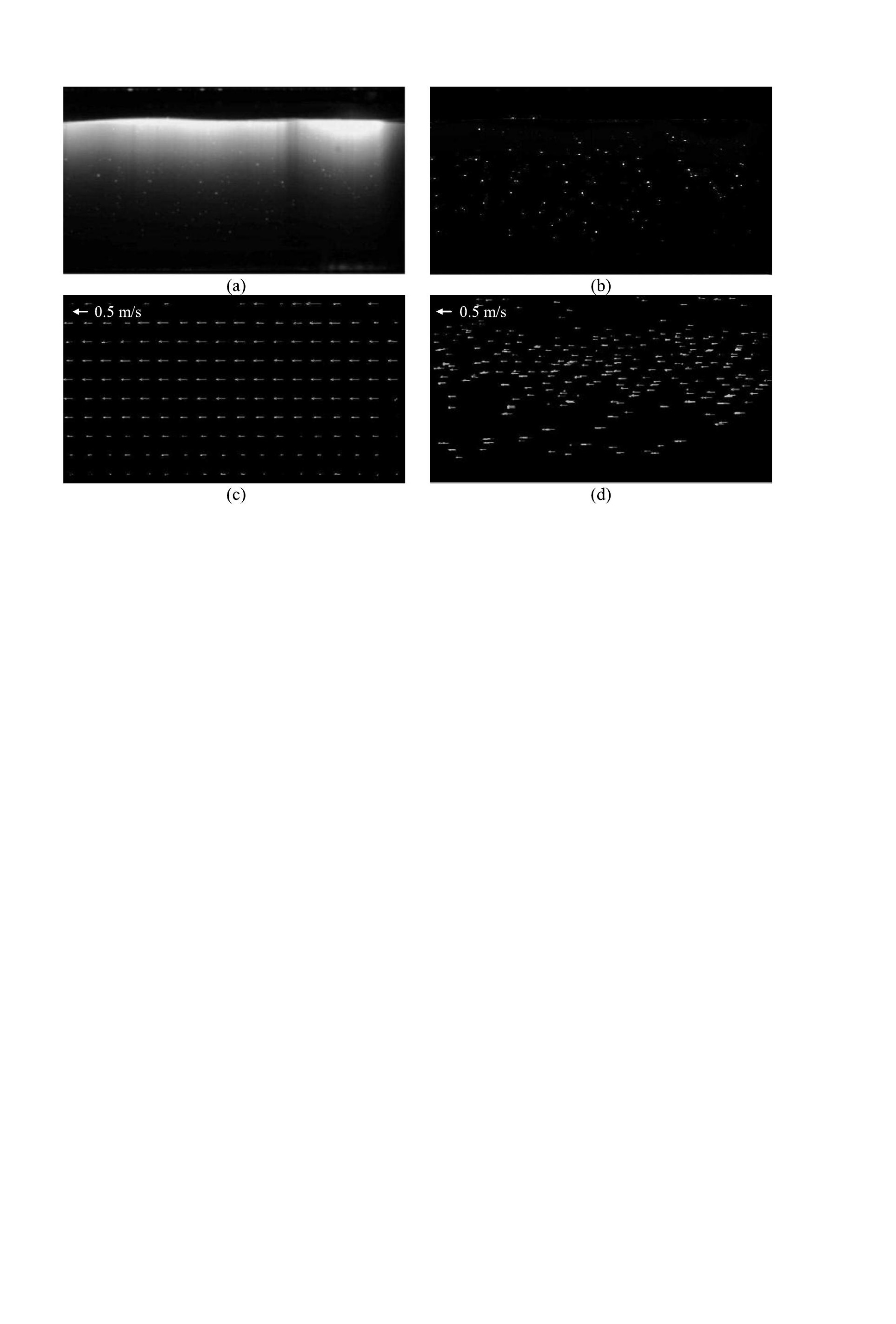

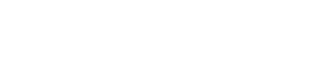
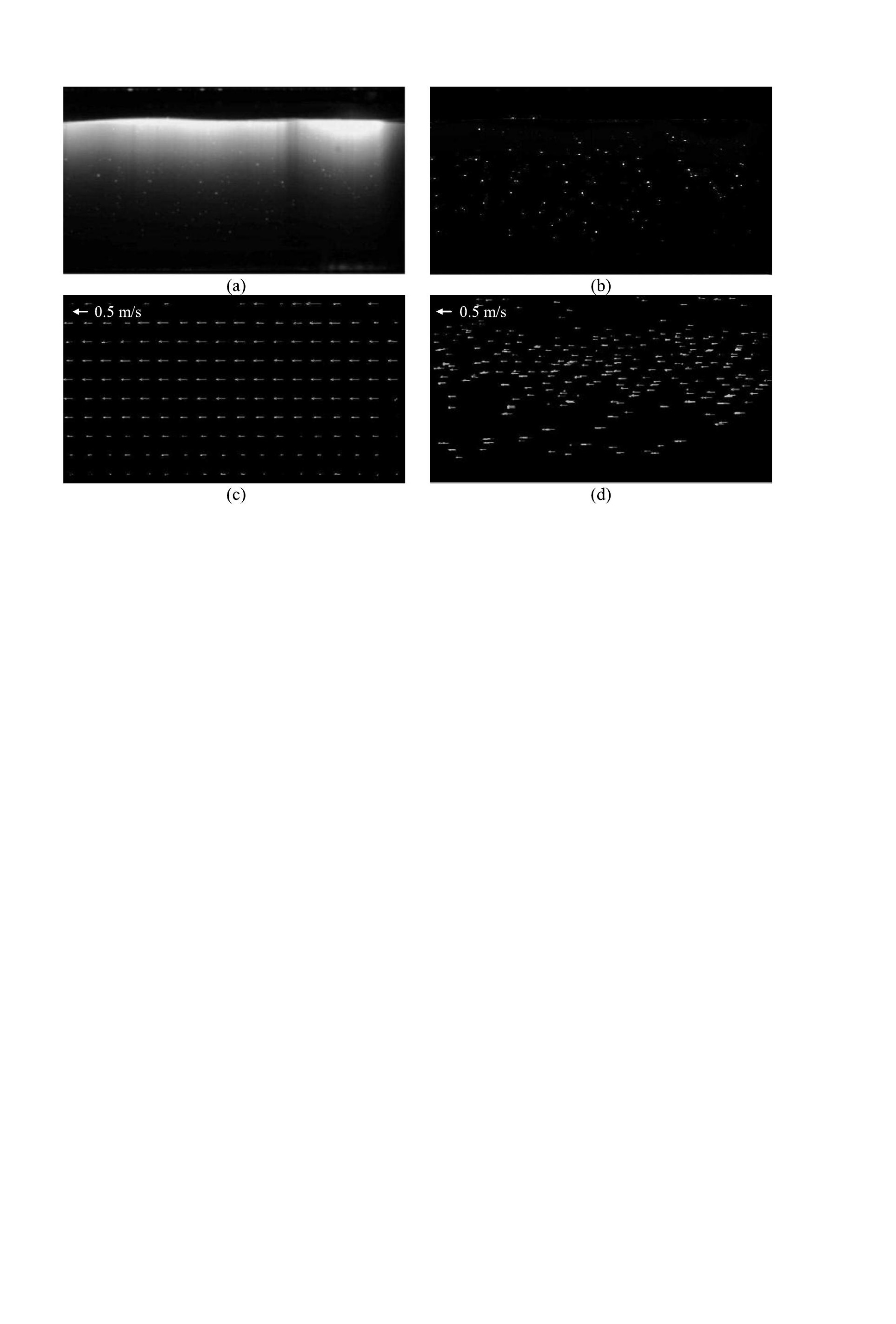

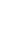$$
\text { (1) }
$$$$
+2
$$$$
\text { (1) }
$$

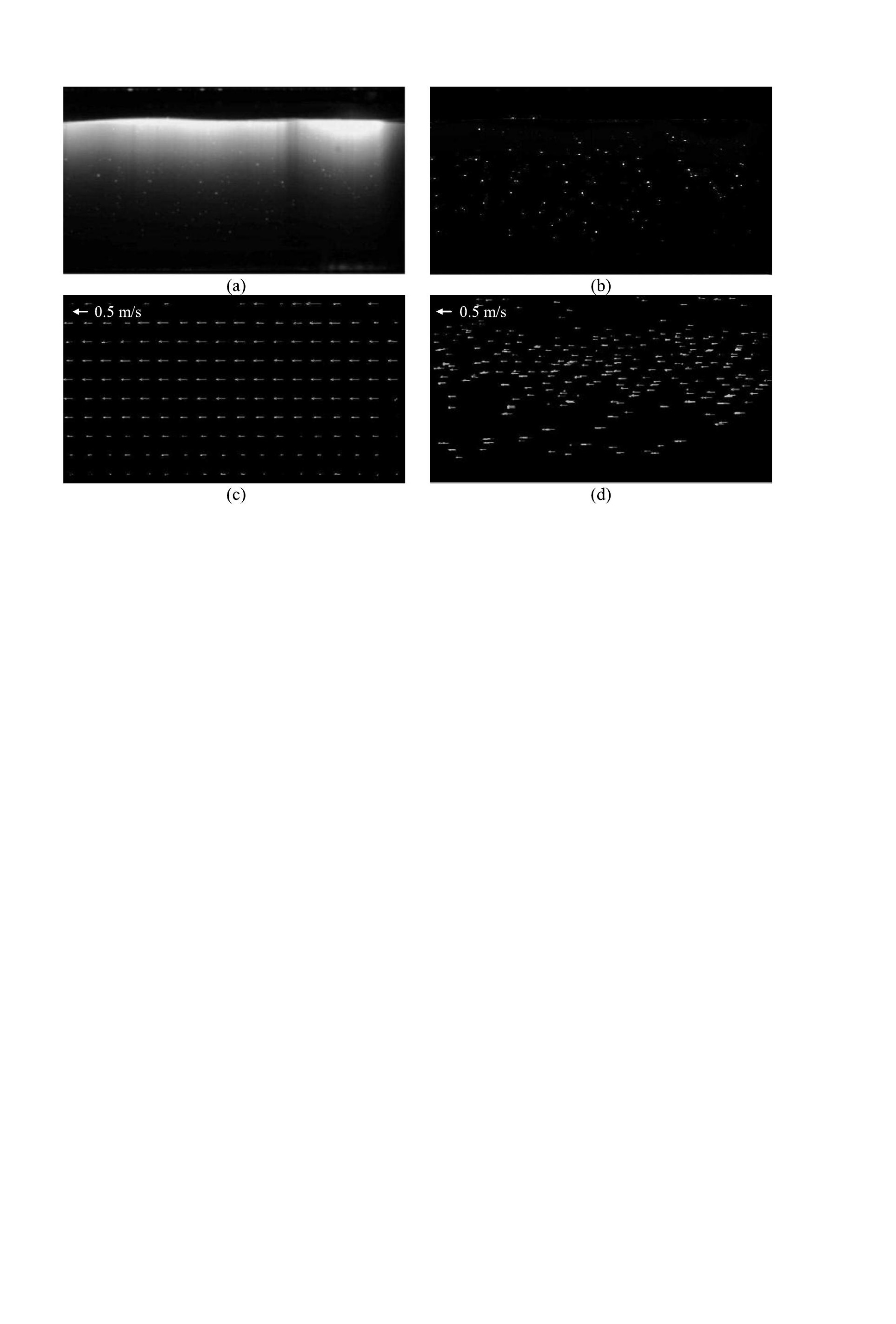$$
\text { (a) }
$$ 


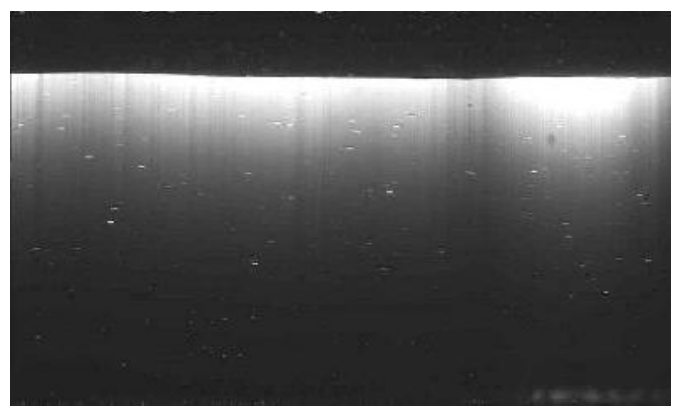

(a) Stratified flow

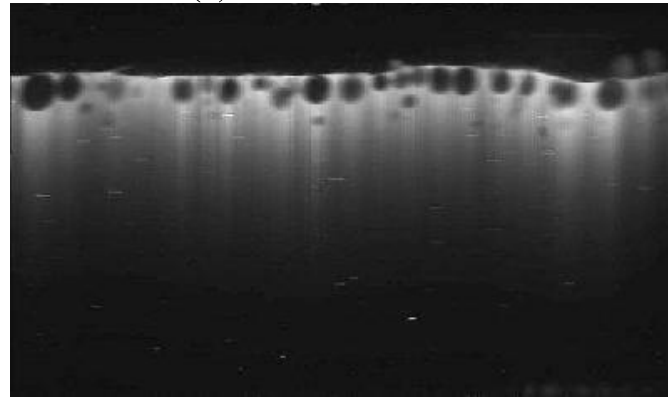

(c) Oil droplet layer

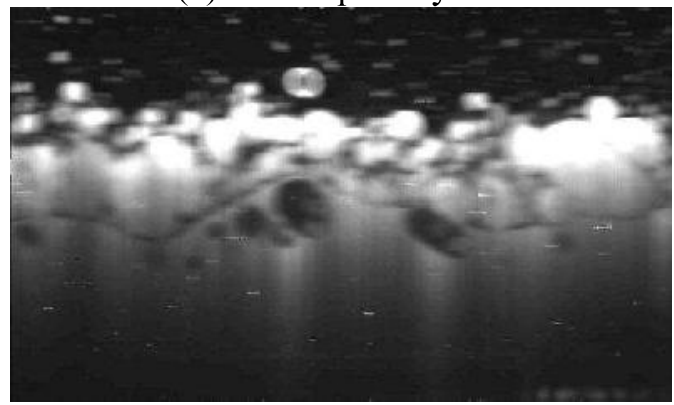

(e) Three layer flow

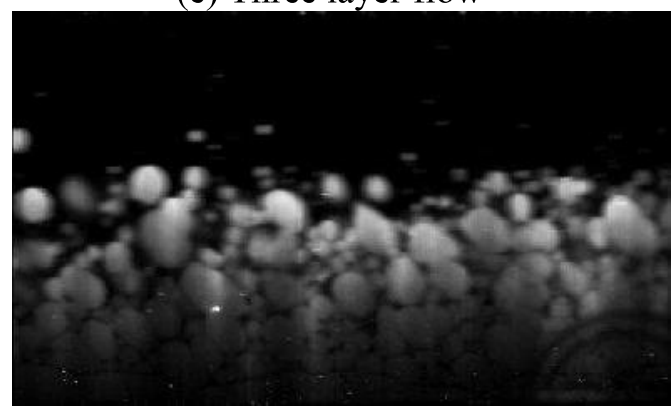

(g) Oil flow over glycerol solution dispersion with glycerol solution film

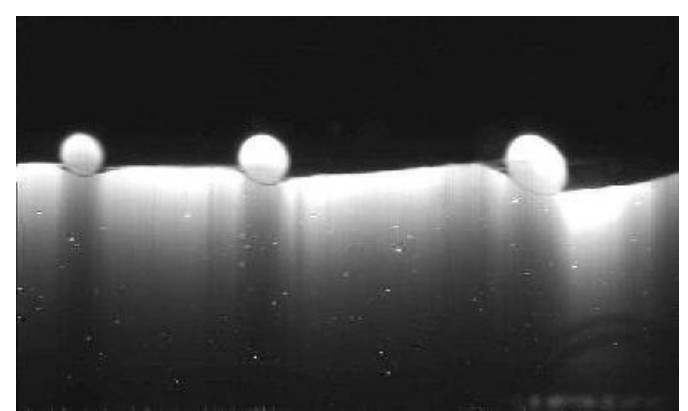

(b) Stratified flow with droplets

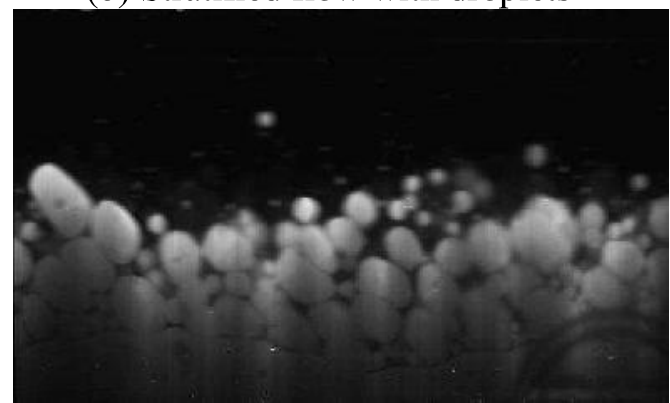

(d) Glycerol solution droplet layer

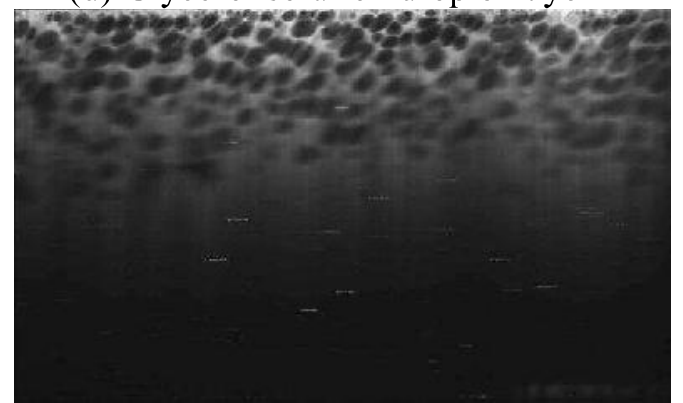

(f) Oil dispersion over glycerol solution

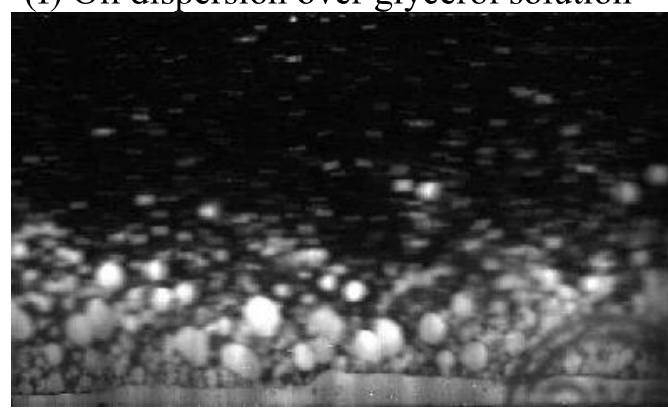

(h) Glycerol solution dispersion with glycerol solution film 


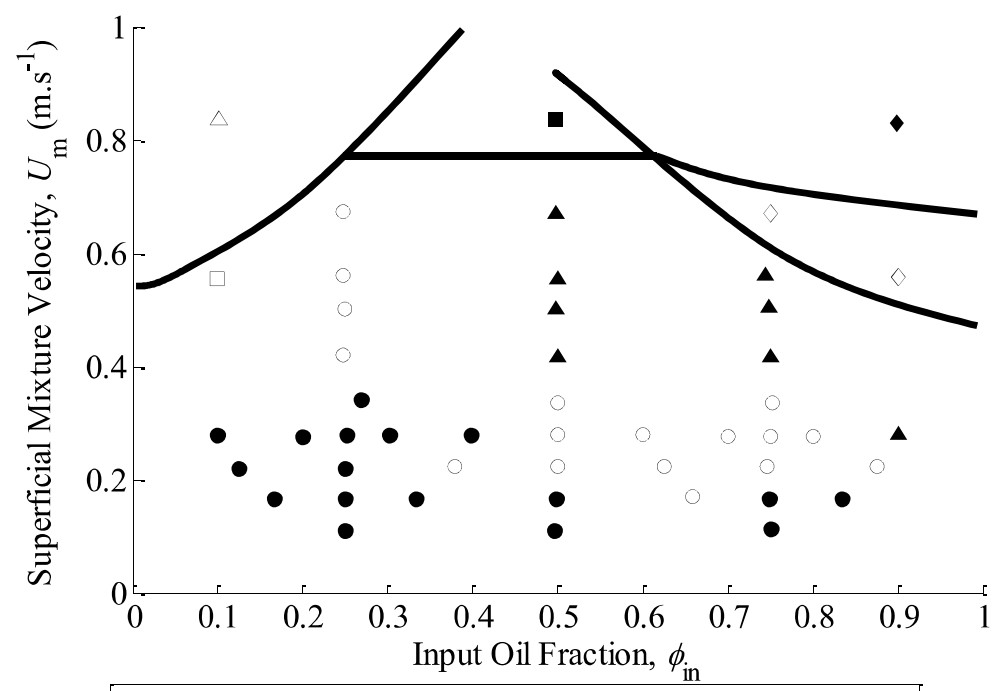

- Stratified Flow

Stratified Flow with Droplets

Oil Droplet Layer

- Three Layer Flow

- Glycerol Solution Droplet Layer

$\triangle$ Oil Dispersion over Glycerol Solution

$\diamond \quad$ Oil Flow over Glycerol Solution Dispersion with Film

- Glycerol Solution Dispersion with Film 


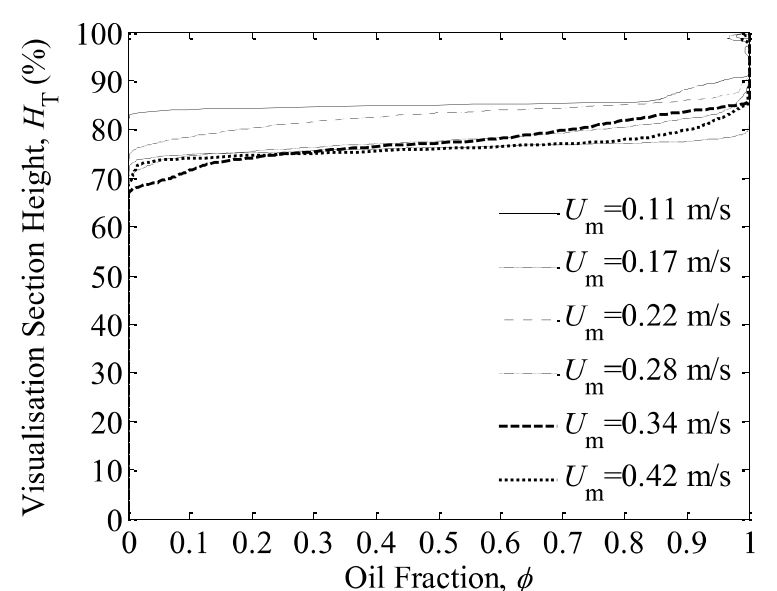

(a)

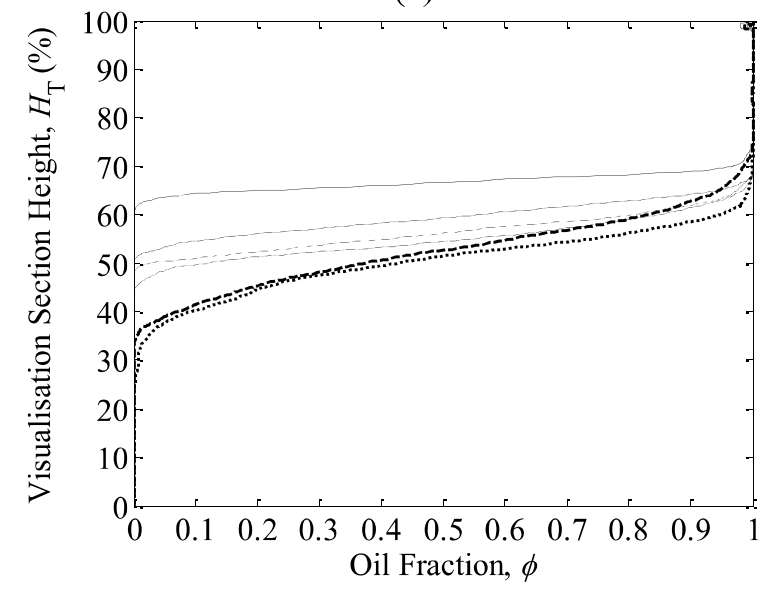

(c)

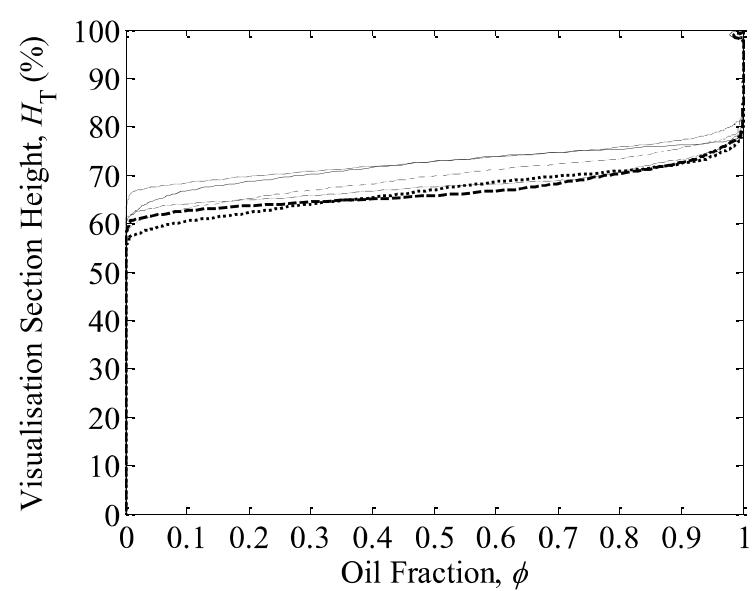

(b)

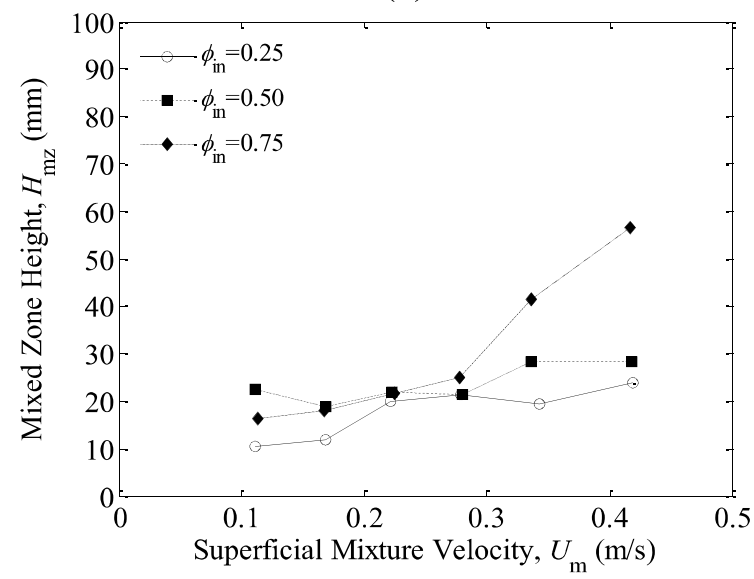

(d) 


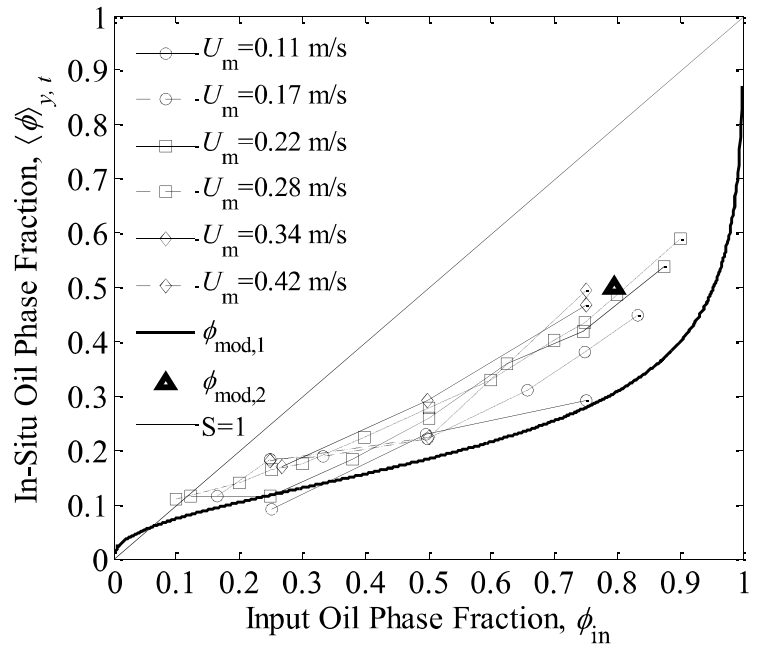


Figure9

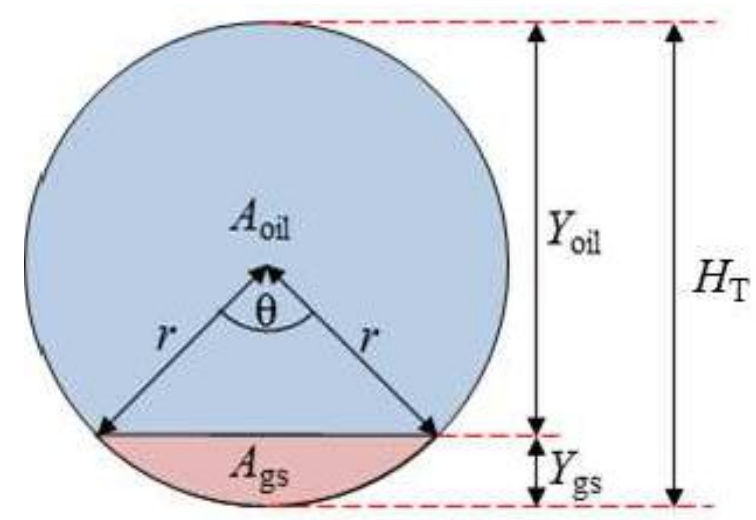

$$
H_{\mathrm{T}}
$$
$\mathrm{T}_{\mathrm{T}}$ 


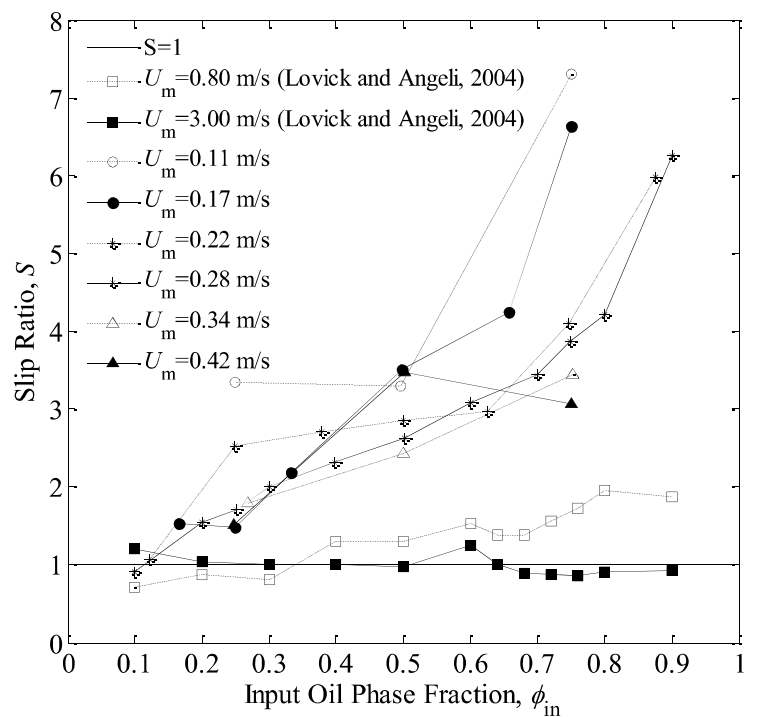




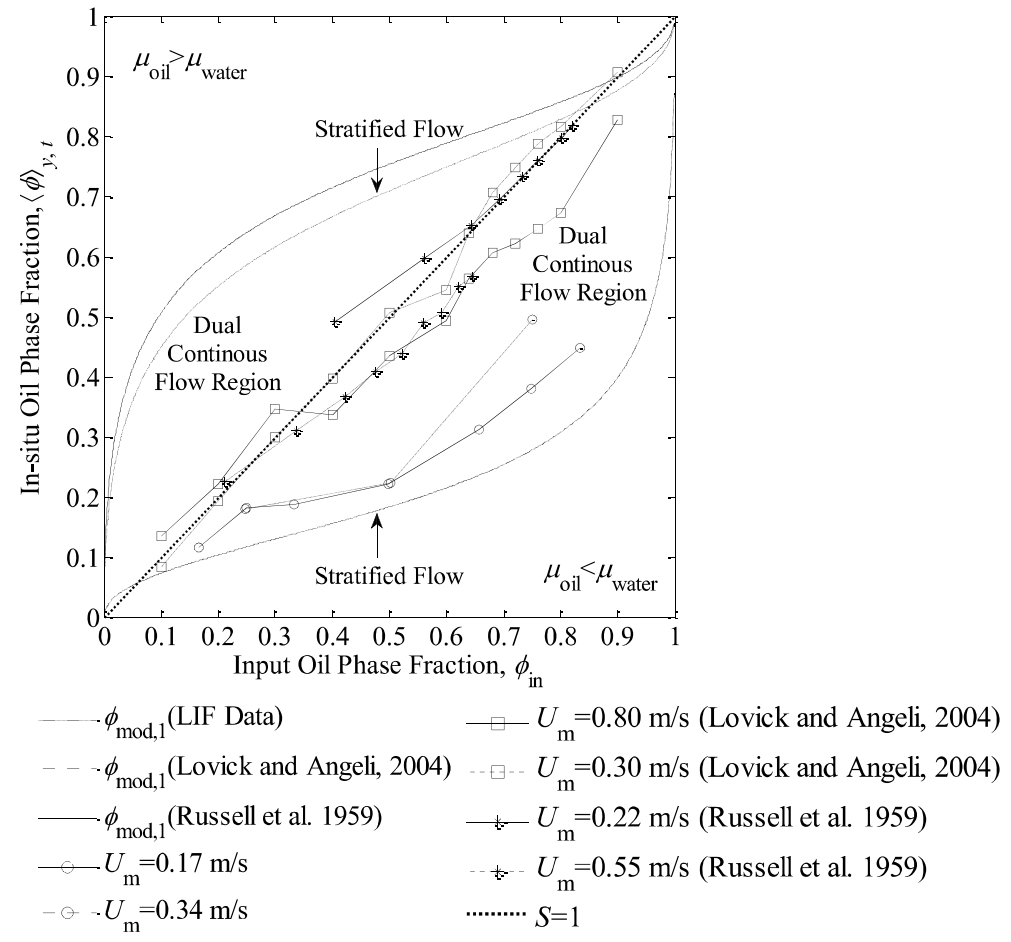




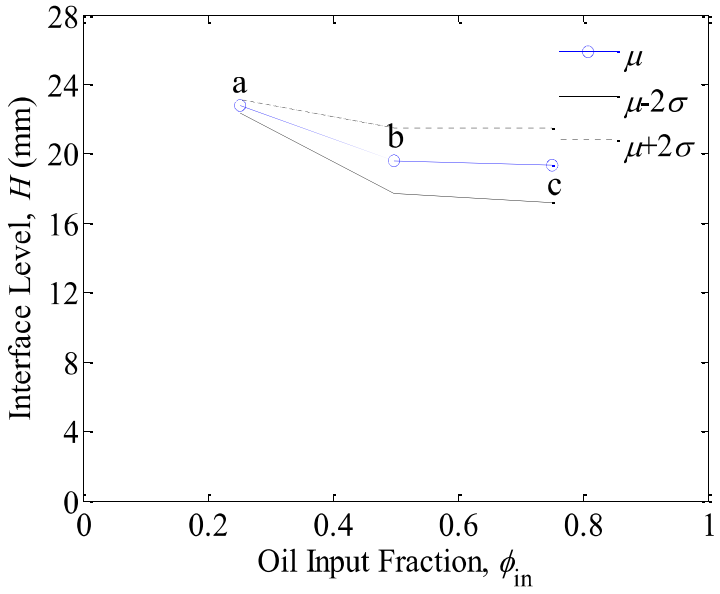

(a)

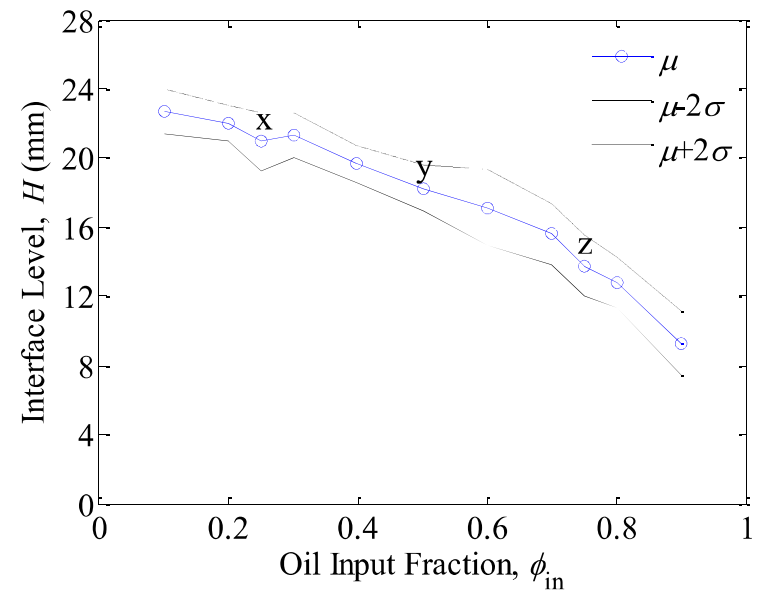

(b) 


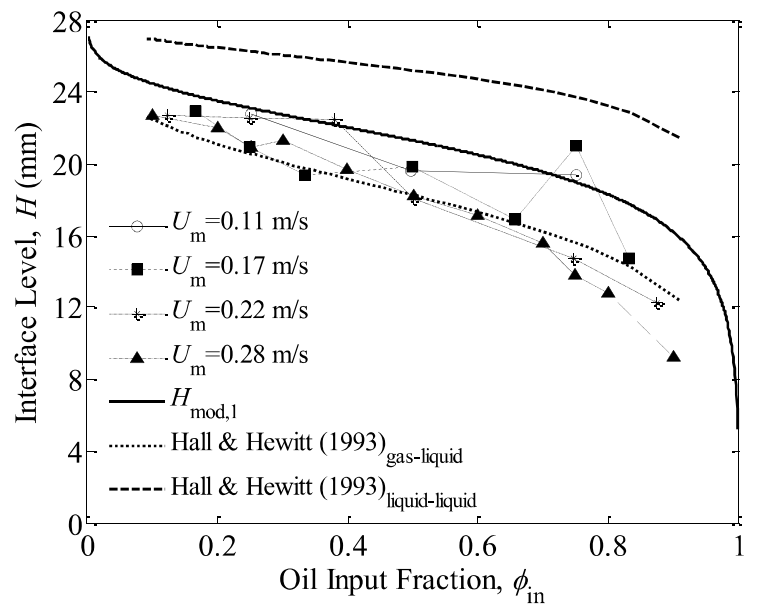




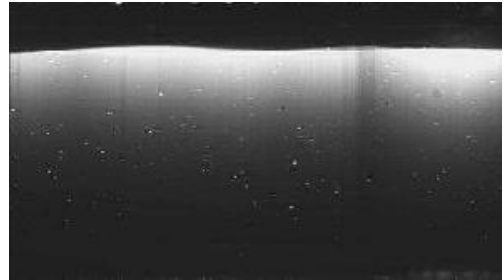

$\left(a_{1}\right)$

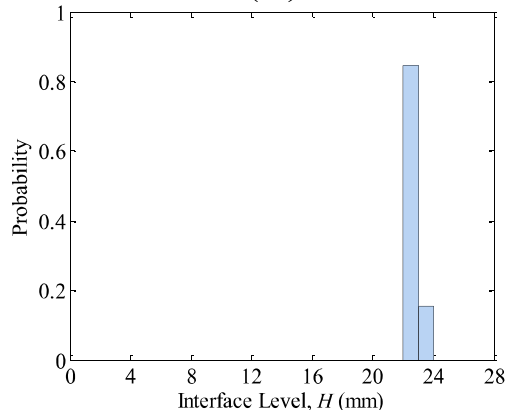

$\left(a_{2}\right)$

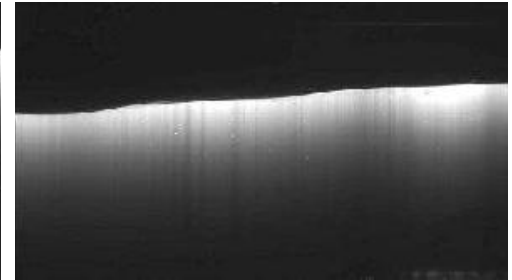

$\left(b_{1}\right)$

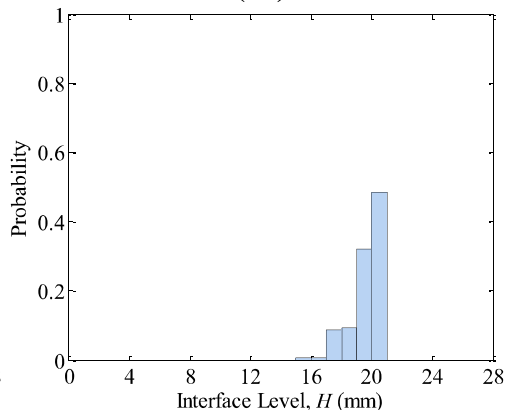

$\left(b_{2}\right)$

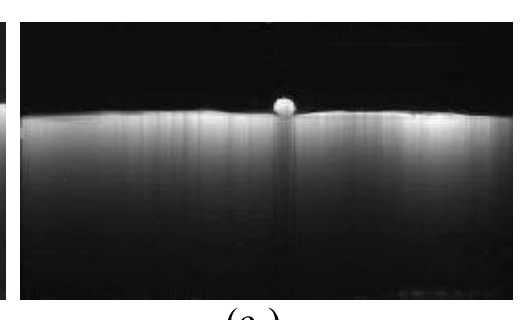

$\left(c_{1}\right)$

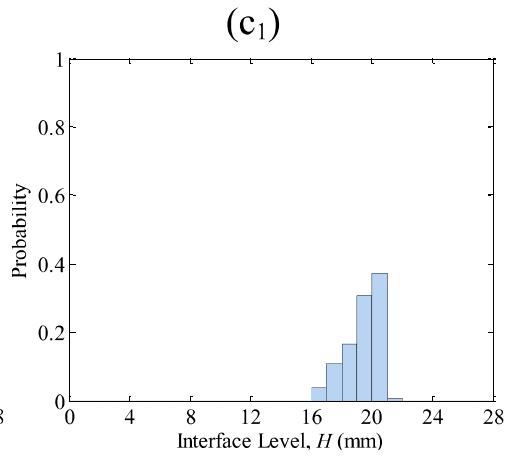

$\left(c_{2}\right)$
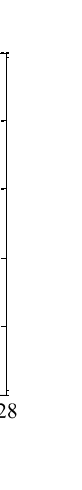
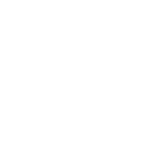


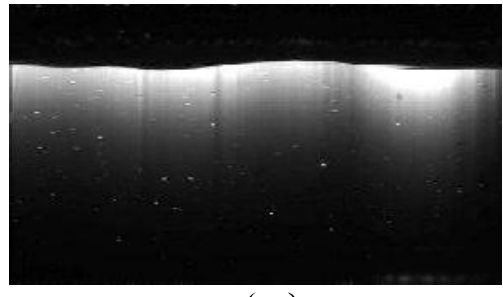

$\left(\mathrm{x}_{1}\right)$

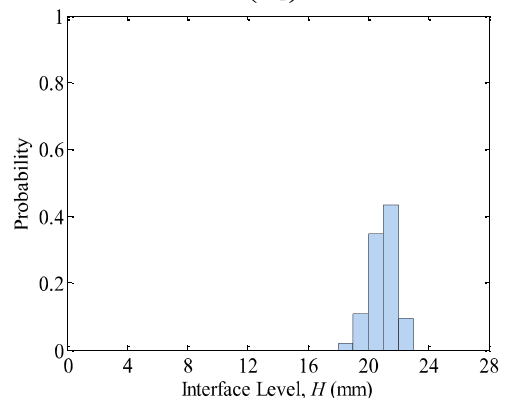

$\left(\mathrm{x}_{2}\right)$

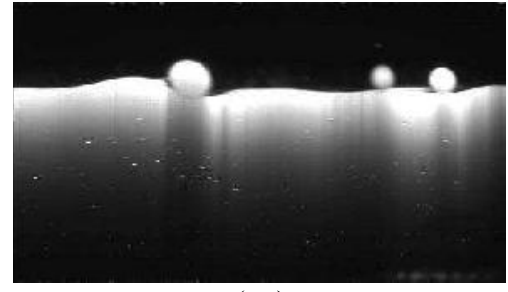

$\left(\mathrm{y}_{1}\right)$

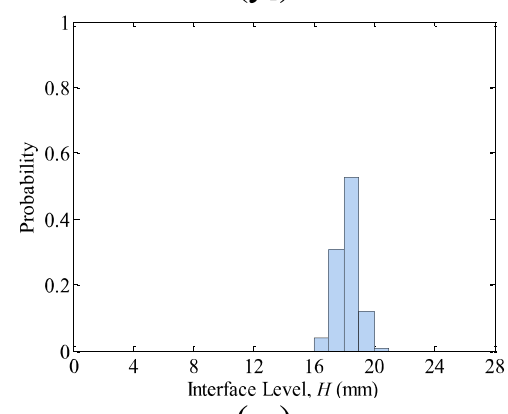

$\left(\mathrm{y}_{2}\right)$

(

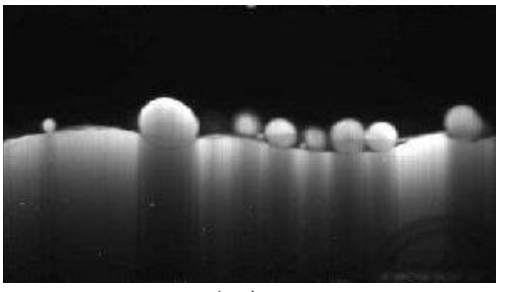

$\left(\mathrm{z}_{1}\right)$

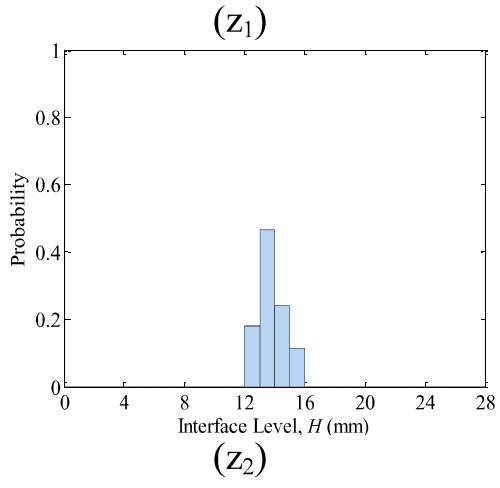

$\left(\mathrm{z}_{2}\right)$ 


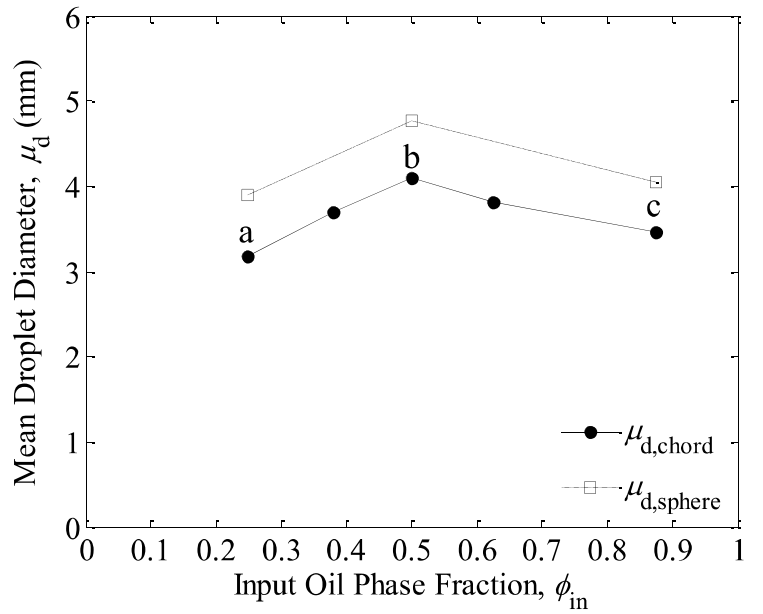

(a)

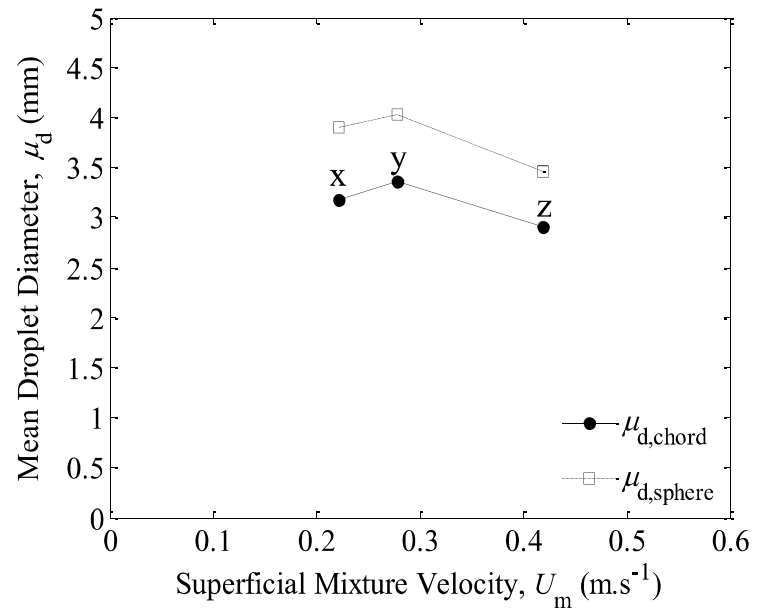

(b) 
$\left(a_{1}\right)$

$\left(a_{2}\right)$
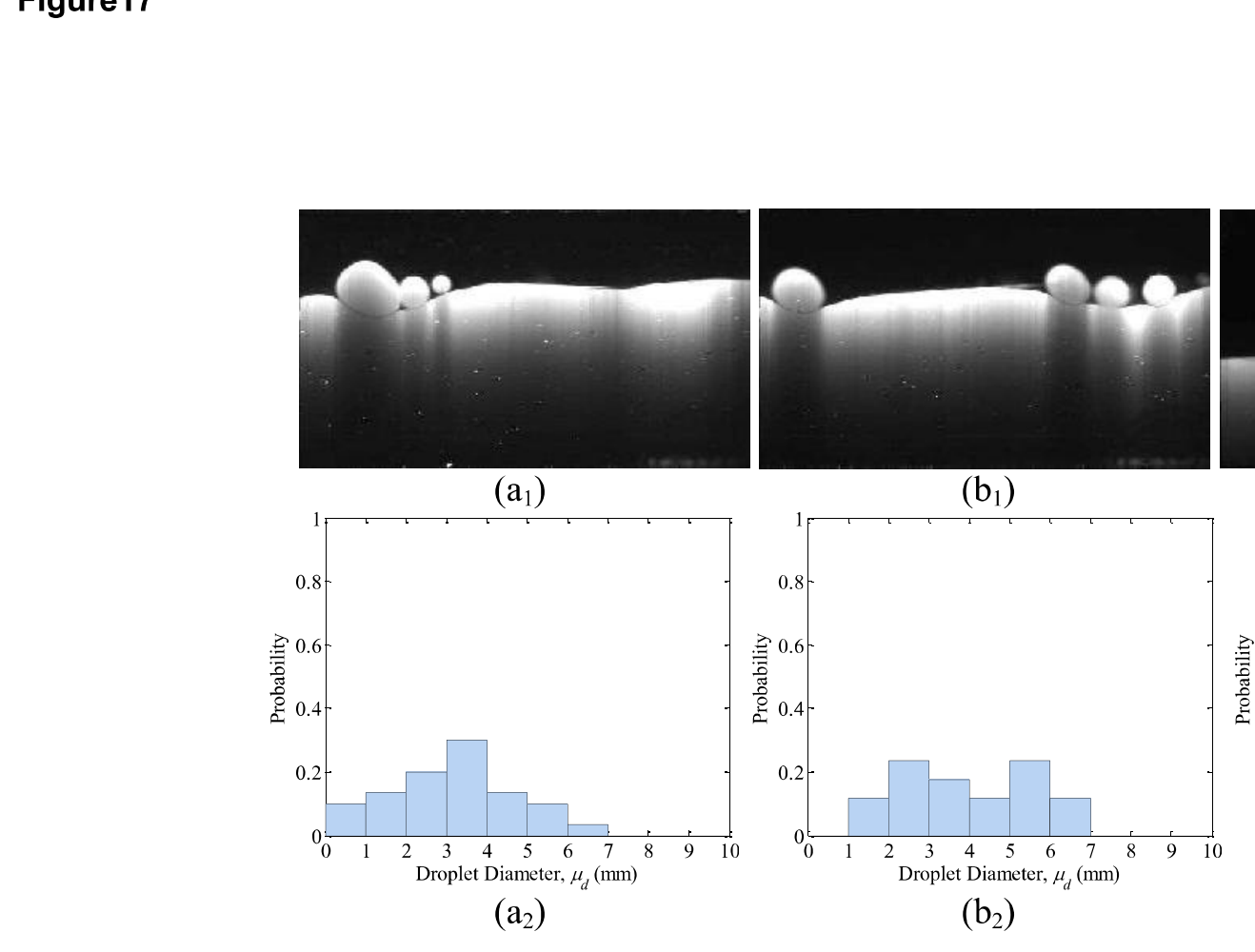

$\left(b_{1}\right)$
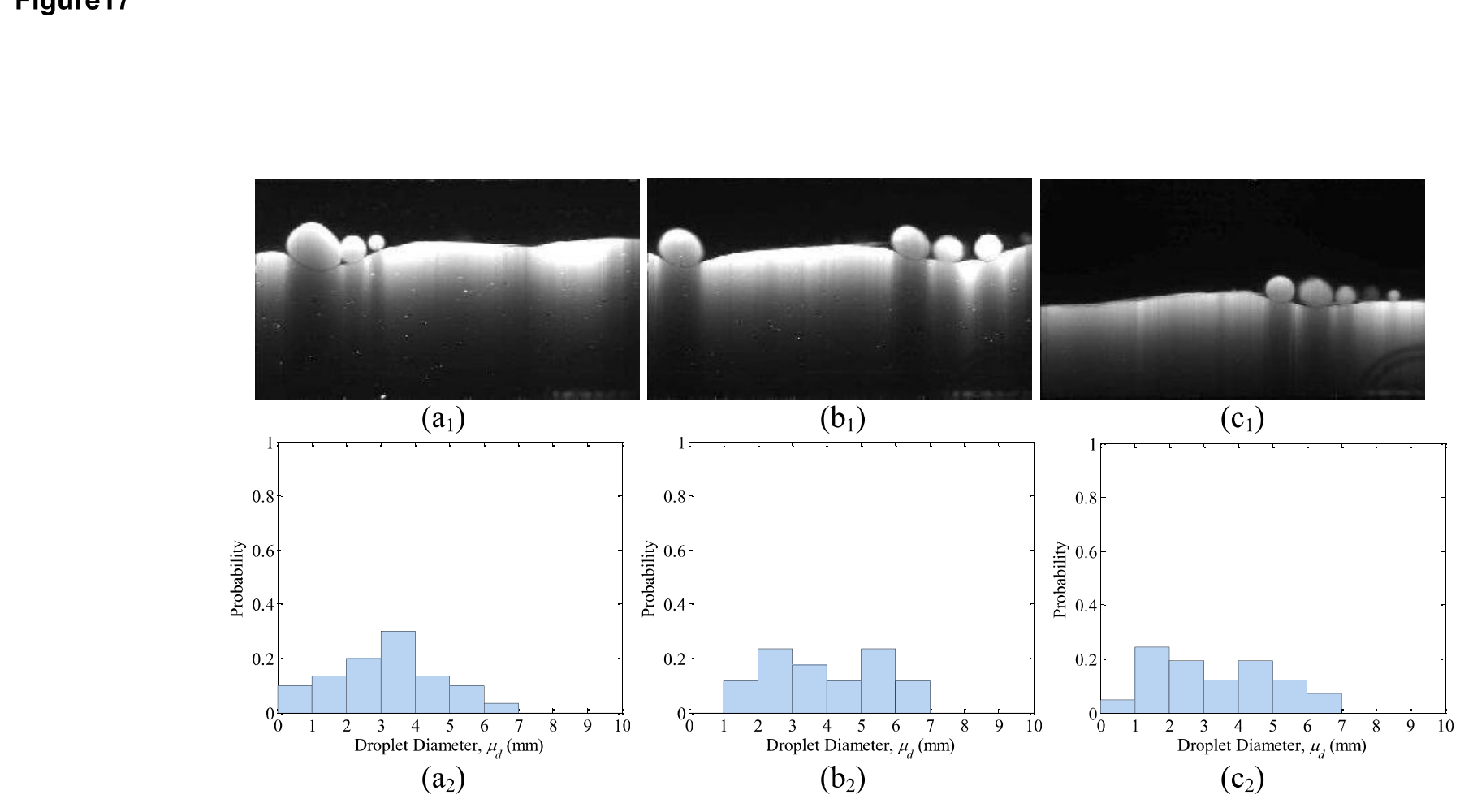

$\left(c_{1}\right)$

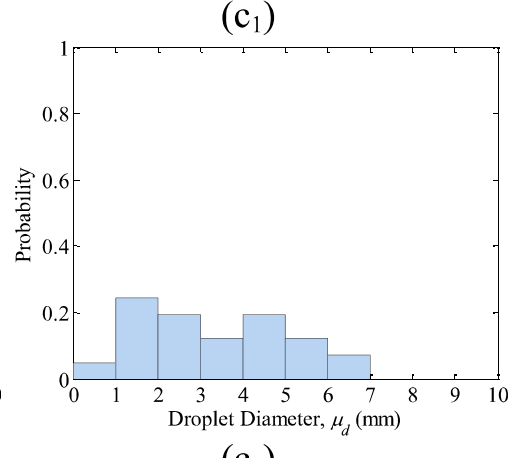

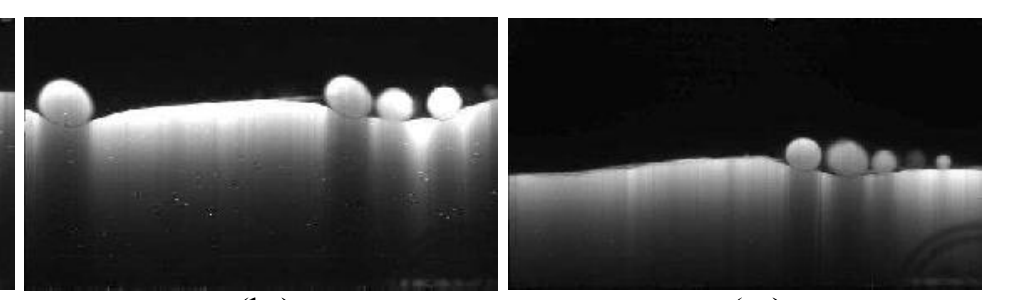

.

.

.

$+2$
$\left(b_{2}\right)$
$\left(c_{2}\right)$
$(-2$
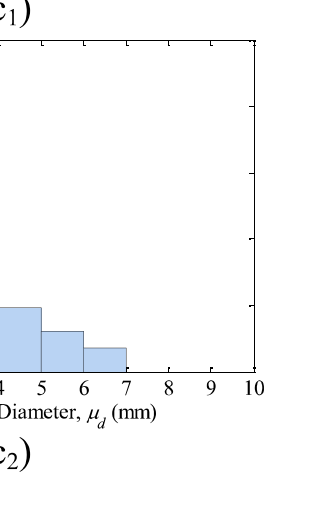

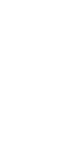
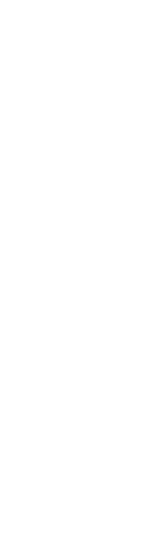


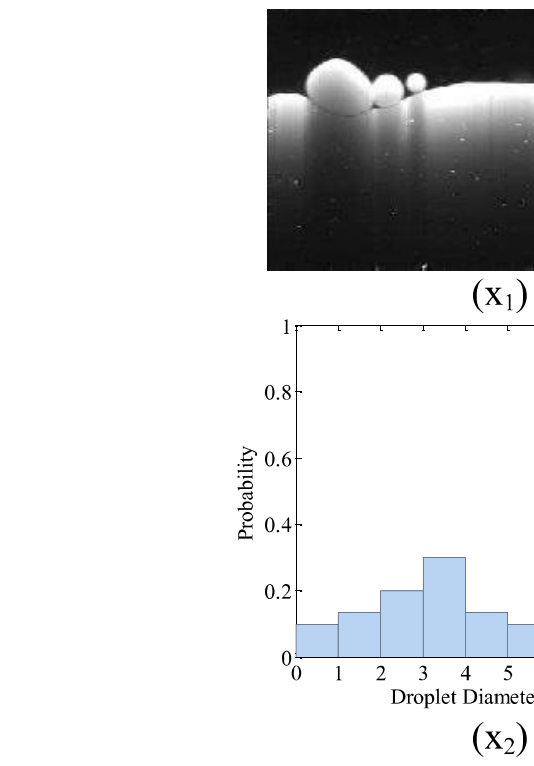

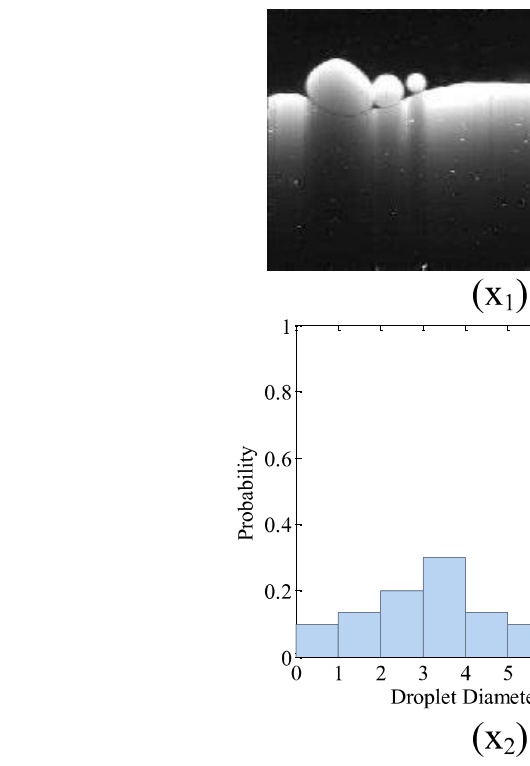

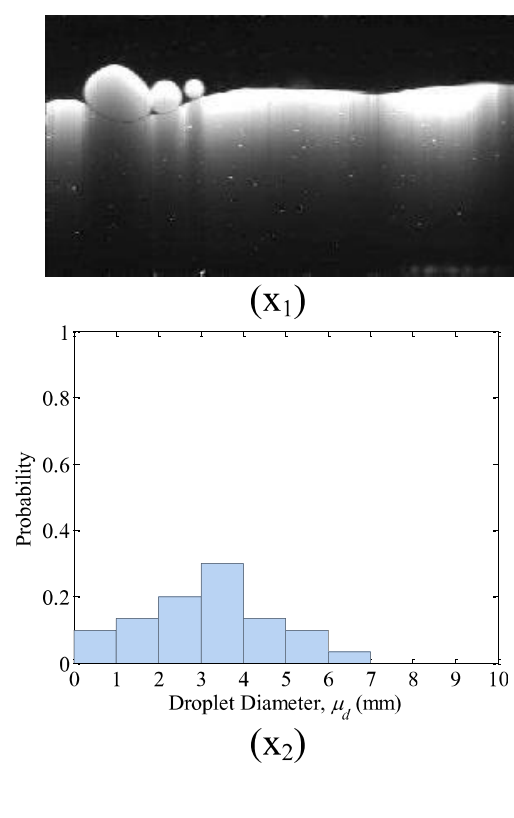

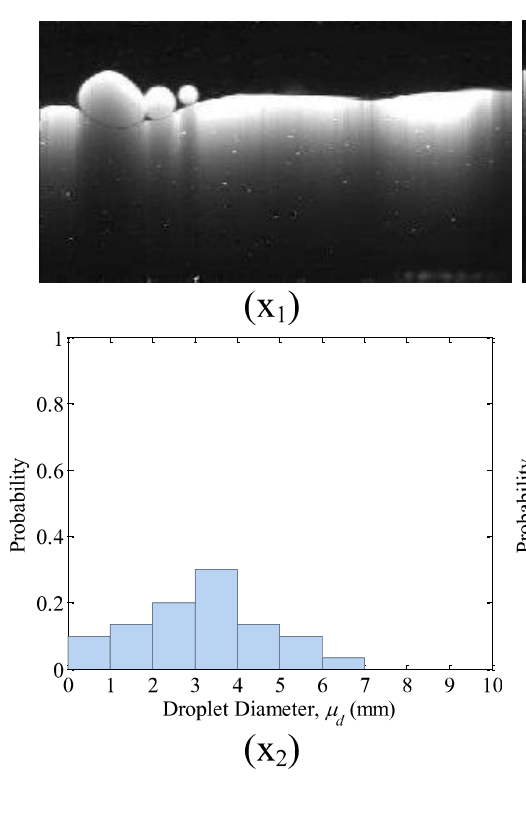

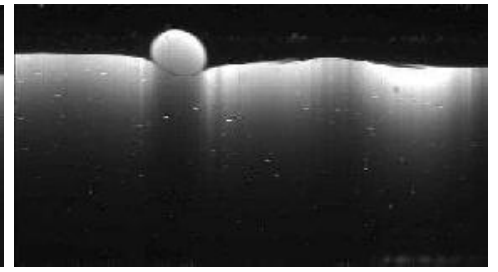

$\left(\mathrm{y}_{1}\right)$

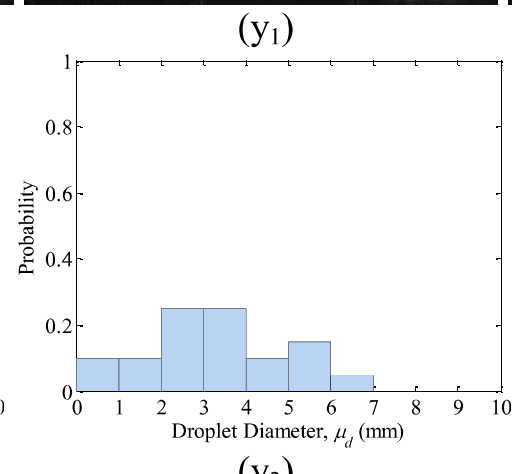

$\left(\mathrm{y}_{2}\right)$ (a)

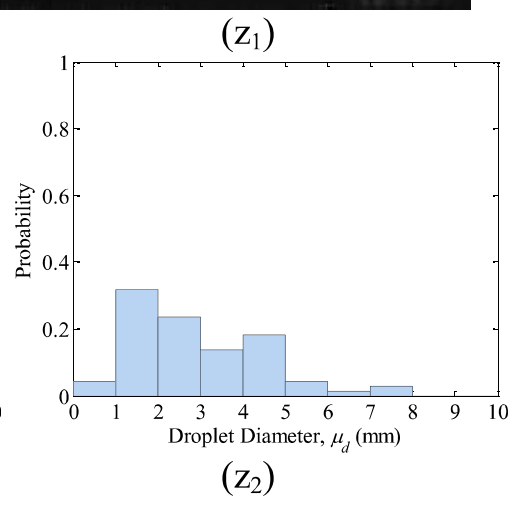

$\left(\mathrm{z}_{1}\right)$

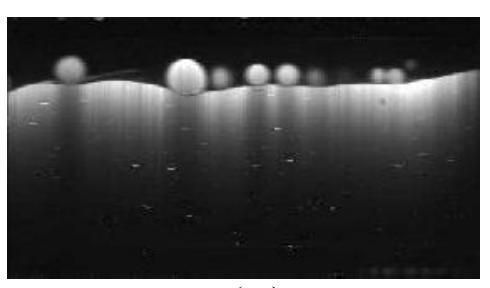

$\left(\mathrm{z}_{2}\right)$
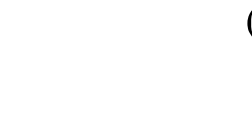

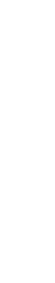

.

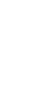




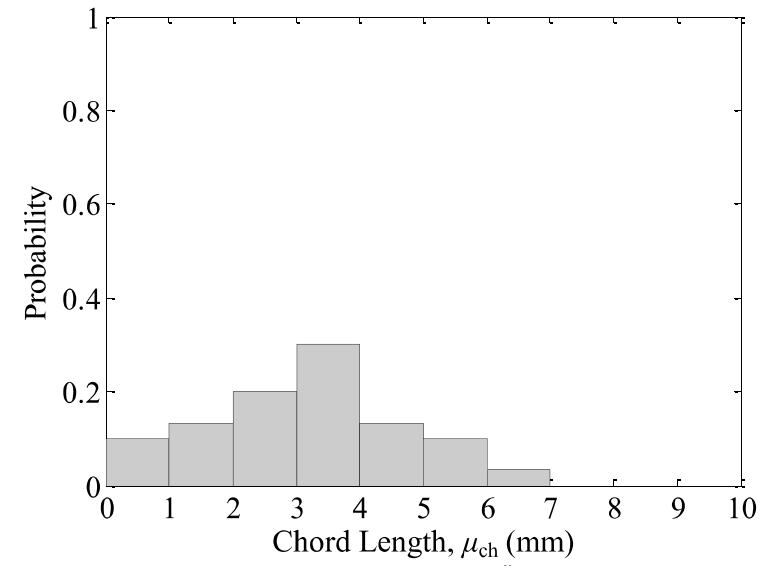

(a)

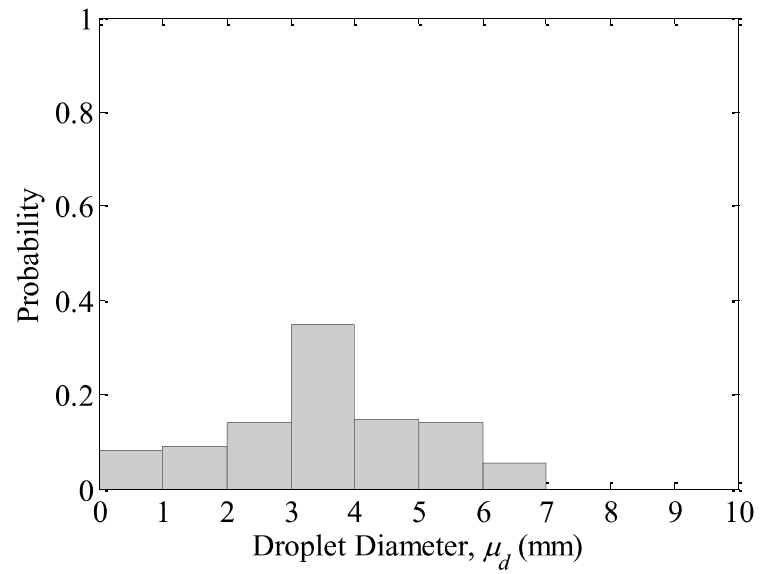

(b) 


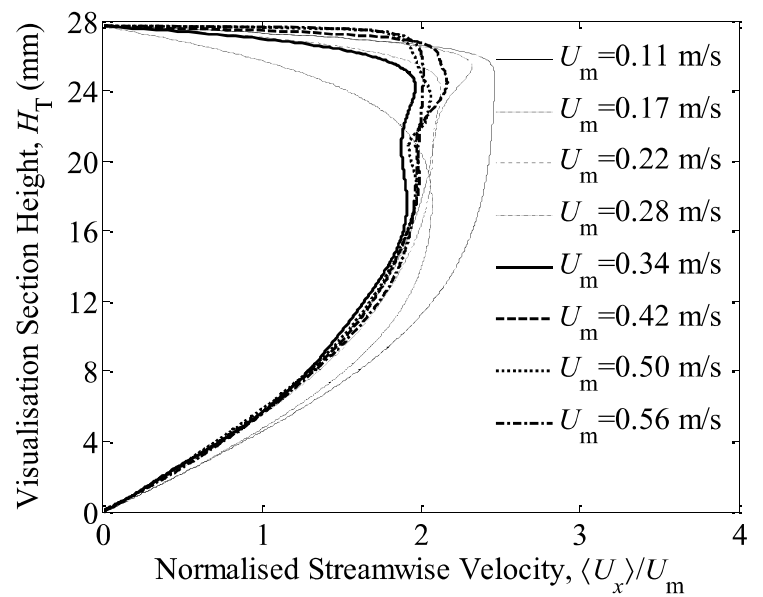

(a)

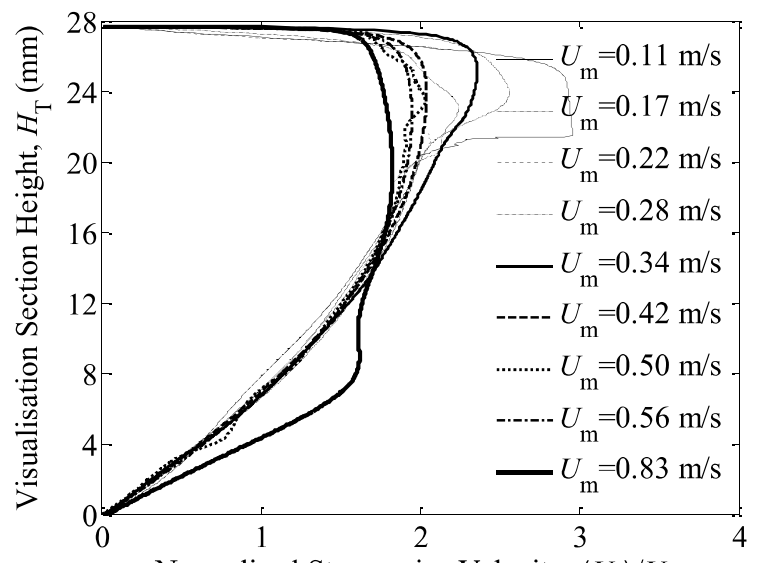

Normalised Streamwise Velocity, $\left\langle U_{x}\right\rangle / U_{\mathrm{m}}$

(b)

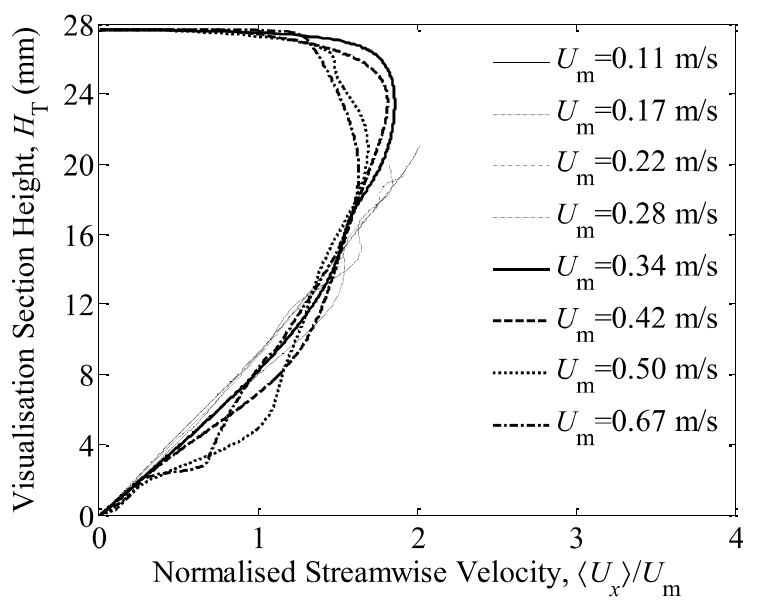

(c) 


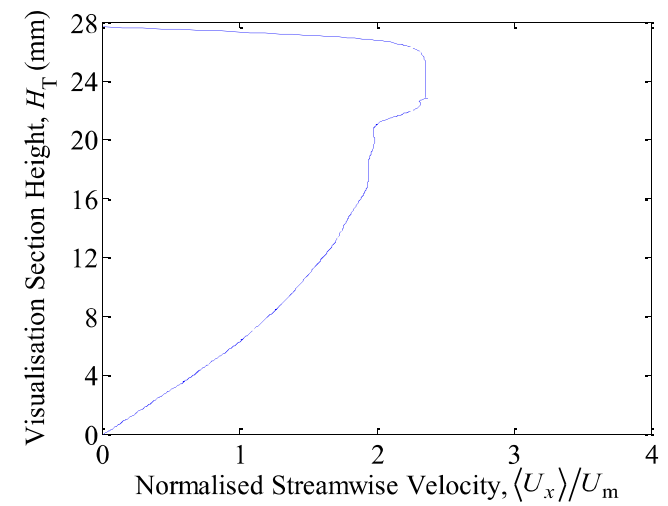

(a)

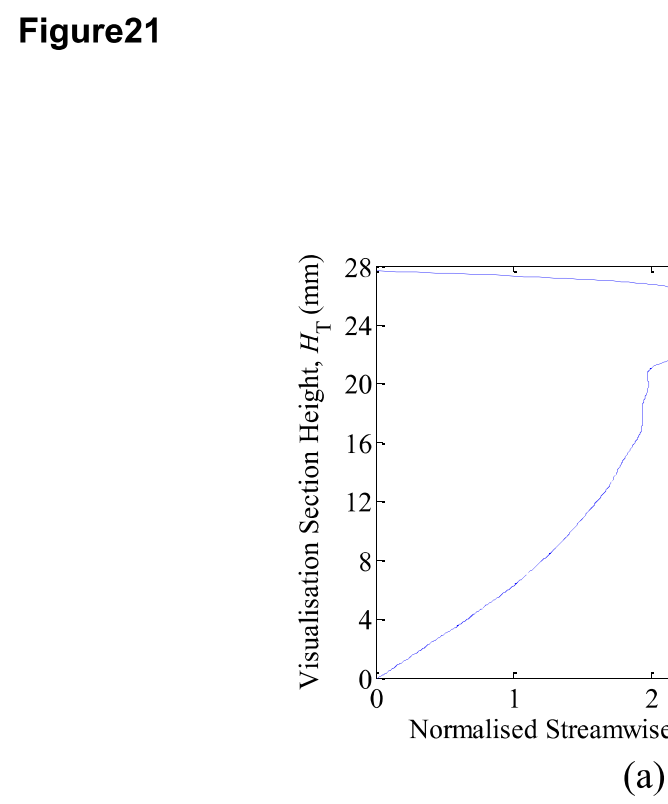

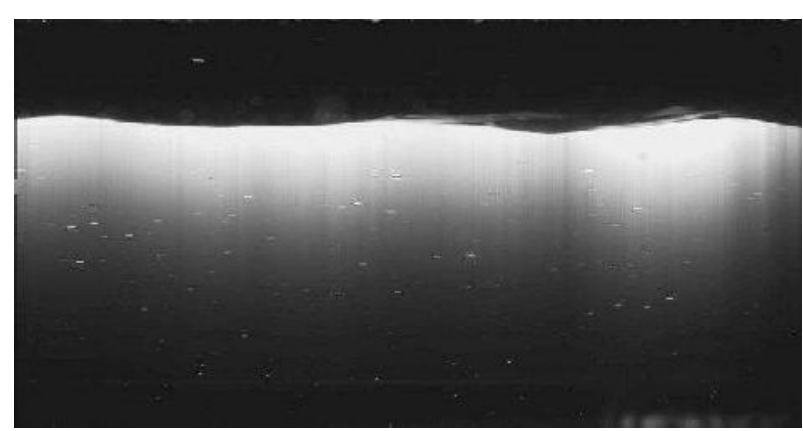

(b) 


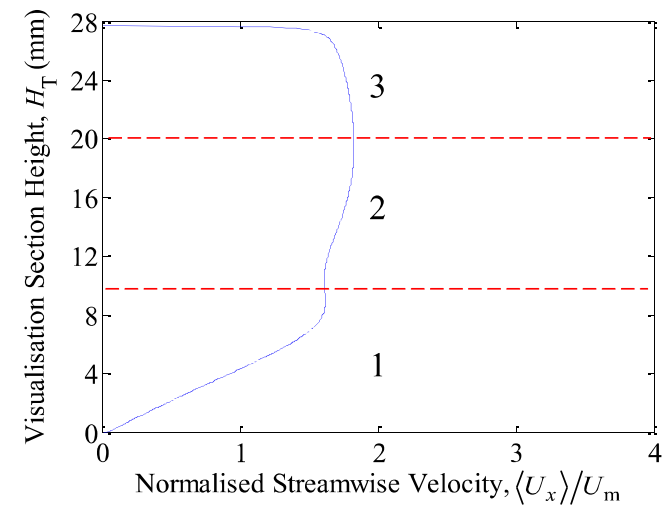

(a)

.

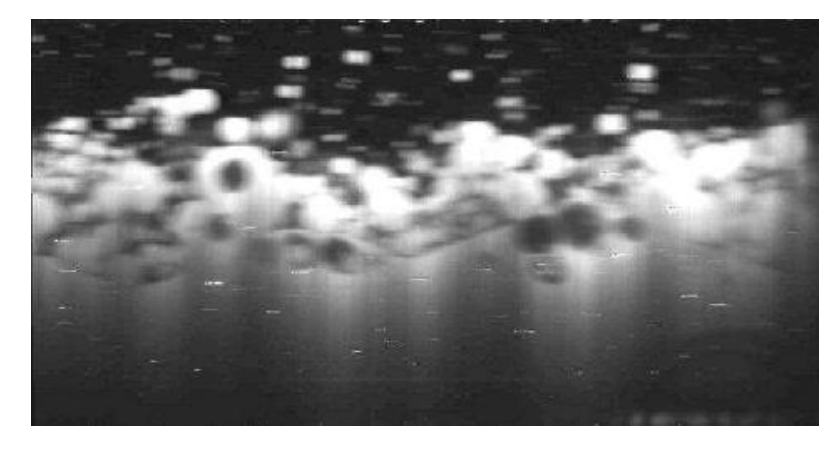

(b) 


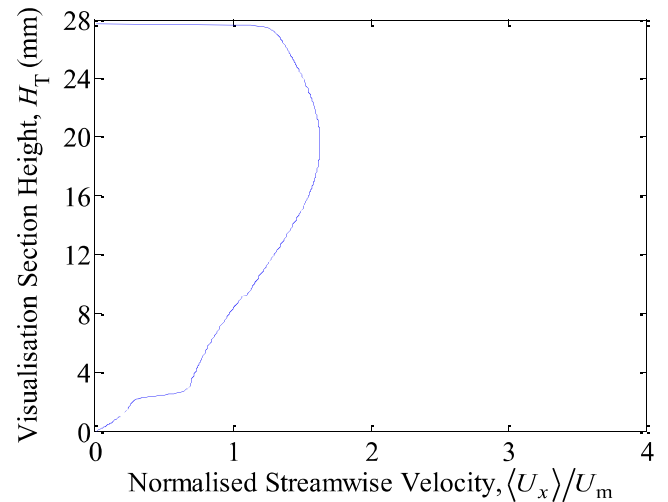

(a1)

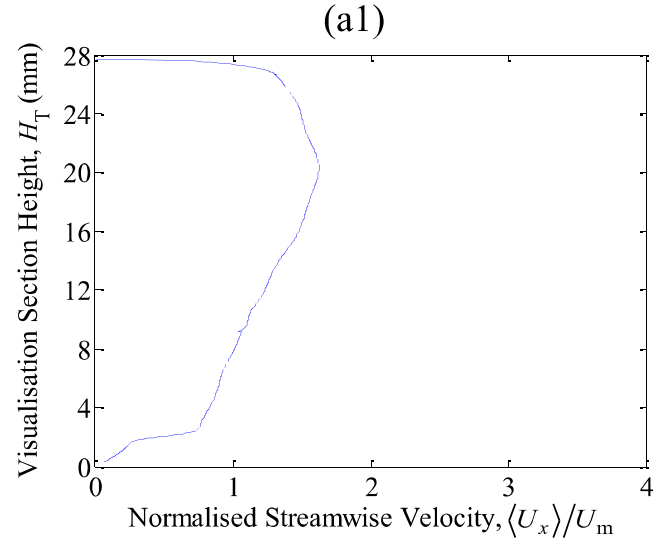

(a2)

(a2)

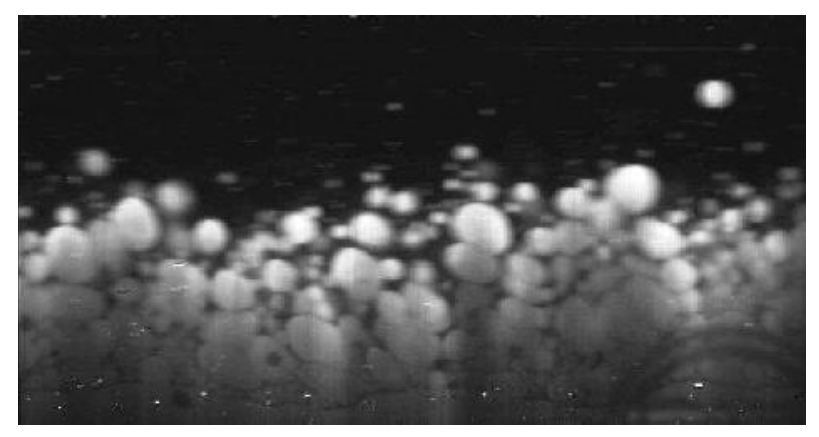

(b1)

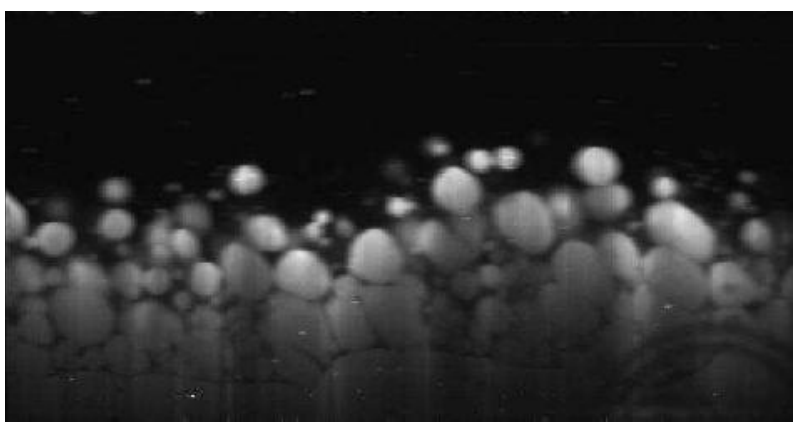

(b2)

(b2) 


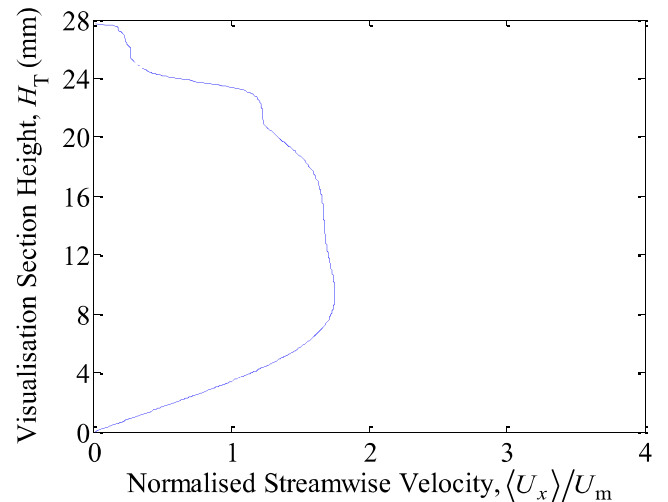

(a1)

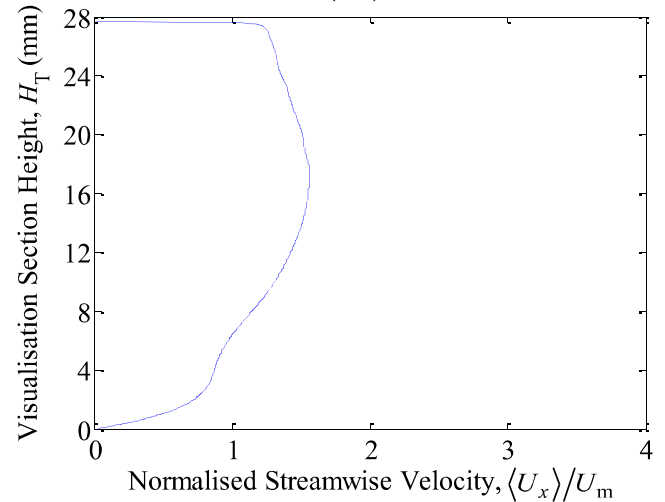

(a2)

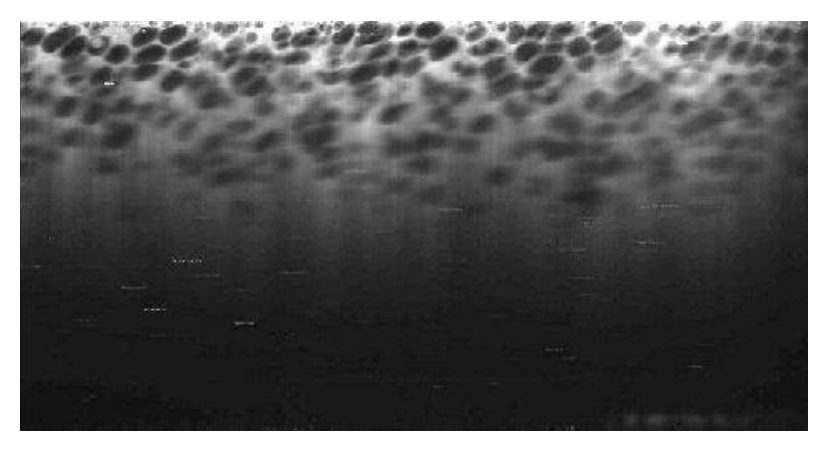

(b1)

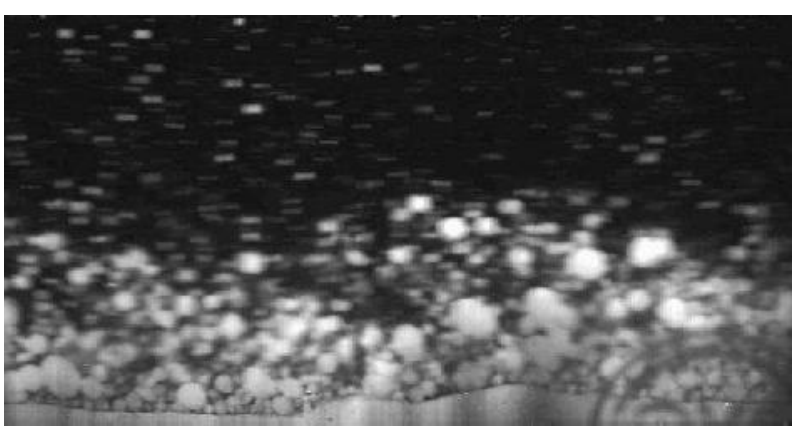

(b2) 


\section{Figure Captions}

Figure 1: Schematic diagram of the TOWER multiphase flow facility.

Figure 2: Circular cross-section visualisation section.

Figure 3: Demonstration of the graticule image correction technique: (a) a raw image of the graticule; (b) the image in Figure 3(a) after it has undergone correction; (c) a raw PLIF image and (d) the image in Figure 3(c) after it has undergone correction.

Figure 4: The PIV/PTV process for acquiring velocity vectors between two images separated by a known time interval: (a) a distortion-corrected image; (b) the image in Figure 4(a) after it has undergone sliding minimum subtraction; (c) a velocity vector map calculated using the PIV technique between two successive images, and; (d) a PTV vector map which is calculated from the PIV vector map shown in Figure 4(c). In Figure 4(c) and 4(d) the size of the vectors (arrows) refers to the velocity.

Figure 5: Typical instantaneous PLIF images of the eight distinct flow regimes observed in the experimental campaign.

Figure 6: Flow regime map constructed from series of flow images such as those in Figure 5.

Figure 7: Vertical time-averaged oil phase fraction profiles $\bar{\phi}(y)$ for different superficial mixture velocities $U_{\mathrm{m}}$ at an input oil fraction $\phi_{\text {in }}$ of: (a) 0.25 , (b) 0.50 , (c) 0.75 , and (d) interface zone height as a function of superficial mixture velocities $U_{\mathrm{m}}$ for constant input oil fractions $\varphi_{\mathrm{in}}$.

Figure 8: In-situ oil phase fraction $\langle\phi\rangle_{y, t}$ as a function of input oil fraction $\phi_{\text {in }}$ for different superficial mixture velocities $U_{\mathrm{m}}$. 
Figure 9: Stratified liquid-liquid flow model construction.

Figure 10: Slip ratio $S$ as a function of input oil fraction $\phi_{\text {in }}$ for different superficial mixture velocities $U_{\mathrm{m}}$. PLIF data is presented in blue and the results from Lovick and Angeli (2004) are presented in red.

Figure 11: In-situ phase fraction $\langle\phi\rangle_{y, t}$ as a function of input oil fraction $\phi_{\text {in }}$ for different superficial mixture velocities $U_{\mathrm{m}}$, compared with data from Lovick and Angeli (2004) and Russell et al. (1959).

Figure 12: The mean $\mu$, upper $\mu+2 \sigma$ and lower $\mu-2 \sigma$ limits for the interface level $H$ as a function of input oil fraction $\phi_{\text {in }}$ for superficial mixture velocities $U_{\mathrm{m}}$ of: (a) $0.11 \mathrm{~m} \cdot \mathrm{s}^{-1}$, and; (b) $0.28 \mathrm{~m} \cdot \mathrm{s}^{-1}$. Points $\mathrm{a}, \mathrm{b}$ and $\mathrm{c}$ correspond to the instantaneous images $\mathrm{a}_{1} \rightarrow \mathrm{c}_{1}$ and probability histograms $\mathrm{a}_{2} \rightarrow \mathrm{c}_{2}$ in Figure 14. Points $\mathrm{x}, \mathrm{y}$ and $\mathrm{z}$ correspond to the instantaneous images $\mathrm{x}_{1} \rightarrow \mathrm{z}_{1}$ and probability histograms $\mathrm{x}_{2} \rightarrow \mathrm{z}_{2}$ in Figure 15 .

Figure 13: Interface level $H$ as a function of input oil fraction $\phi_{\text {in }}$ for different superficial mixture velocities $U_{\mathrm{m}}$ featuring interface level models presented by Hall and Hewitt (1993) for stratified liquid-liquid flow, stratified gas-liquid flow and the laminar drag model, $H_{\mathrm{mod}, 1}$.

Figure 14: Flow images with input oil fraction $\phi_{\text {in }}$ of: $\left(a_{1}\right) 0.25,\left(b_{1}\right) 0.50,\left(c_{1}\right) 0.75$, all at superficial mixture velocities $U_{\mathrm{m}}=0.11 \mathrm{~m} \cdot \mathrm{s}^{-1} ;\left(\mathrm{a}_{2}\right),\left(\mathrm{b}_{2}\right)$ and $\left(\mathrm{c}_{2}\right)$ show the probability histograms for the same conditions respectively. Data corresponds to Points a, b and c, as labelled in Figure 12.

Figure 15: Flow images with input oil fraction $\phi_{\text {in }}$ of: $\left(\mathrm{x}_{1}\right) 0.25 \mathrm{~m} \cdot \mathrm{s}^{-1},\left(\mathrm{y}_{1}\right) 0.50,\left(\mathrm{z}_{1}\right) 0.75$, all at a superficial mixture velocities $U_{\mathrm{m}}=0.28 \mathrm{~m} \cdot \mathrm{s}^{-1} ;\left(\mathrm{x}_{2}\right),\left(\mathrm{y}_{2}\right)$ and $\left(\mathrm{z}_{2}\right)$ show the probability 
histograms for the same conditions respectively. Data corresponds to Points $\mathrm{x}, \mathrm{y}$ and $\mathrm{z}$, as labelled in Figure 12.

Figure 16: Mean glycerol solution droplet diameter $\mu_{d, \mathrm{gs}}$ for: (a) varying input oil fraction $\varphi_{\text {in }}$ and constant superficial mixture velocities $U_{\mathrm{m}}=0.22 \mathrm{~m} \cdot \mathrm{s}^{-1}$, and; (b) varying superficial mixture velocity $U_{\mathrm{m}}$ and a constant input oil fraction of $\phi_{\mathrm{in}}=0.25$. Points $\mathrm{a}, \mathrm{b}$ and $\mathrm{c}$ correspond to the instantaneous images $a_{1} \rightarrow c_{1}$ and probability histograms $a_{2} \rightarrow c_{2}$ in Figure 17. Points $x, y$ and $z$ correspond to the instantaneous images $x_{1} \rightarrow z_{1}$ and probability histograms $x_{2} \rightarrow z_{2}$ in Figure 18.

Figure 17: Flow images with a superficial mixture velocity $U_{\mathrm{m}}=0.22 \mathrm{~m} \cdot \mathrm{s}^{-1}$ at oil input fractions $\phi_{\text {in }}$ of: $\left(\mathrm{a}_{1}\right) 0.25,\left(\mathrm{~b}_{1}\right) 0.50$ and, $\left(\mathrm{c}_{1}\right) 0.87 ;\left(\mathrm{a}_{2}\right),\left(\mathrm{b}_{2}\right)$ and $\left(\mathrm{c}_{2}\right)$ show the glycerol solution droplet size distribution probability histograms for the same conditions respectively. Data corresponds to Points a, b and c, as labelled in Figure 16(a).

Figure 18: Flow images with an oil input fraction fractions $\phi_{\text {in }}=0.25$ at superficial mixture velocities $U_{\mathrm{m}}$ of: $\left(\mathrm{x}_{1}\right) 0.22,\left(\mathrm{y}_{1}\right) 0.28$ and, $\left(\mathrm{z}_{1}\right) 0.42 ;\left(\mathrm{x}_{2}\right),\left(\mathrm{y}_{2}\right)$ and $\left(\mathrm{z}_{2}\right)$ show the glycerol solution droplet size distribution probability histograms for the same conditions respectively. Data corresponds to Points $\mathrm{x}, \mathrm{y}$ and z, as labelled in Figure 16(b).

Figure 19: Droplet size probability histogram distributions for a flow with a superficial mixture velocity $U_{\mathrm{m}}=0.22 \mathrm{~m} \cdot \mathrm{s}^{-1}$ and at oil input fraction $\phi_{\mathrm{in}}=0.25$ for: (a) chord length, and; (b) droplet diameter.

Figure 20: Normalised velocity profiles $\left\langle U_{x}\right\rangle / U_{\mathrm{m}}$ for different superficial mixture velocities $U_{\mathrm{m}}$ at an input oil fraction $\phi_{\text {in }}$ of: (a) 0.25 ; (b) 0.50 , and; (c) 0.75 . 
Figure 21: (a) Velocity profile, and; (b) instantaneous image, for $U_{\mathrm{m}}=0.28 \mathrm{~m} \cdot \mathrm{s}^{-1}$ and $\phi_{\text {in }}=0.38$.

Figure 22: (a) Velocity profile, and; (b) instantaneous flow image, for three-layer flow with droplets at $U_{\mathrm{m}}=0.84 \mathrm{~m} \cdot \mathrm{s}^{-1}$ and $\phi_{\text {in }}=0.50$.

Figure 23: Velocity profiles $\left(a_{1} \rightarrow a_{2}\right)$ and instantaneous images $\left(b_{1} \rightarrow b_{2}\right)$ for: (1) $U_{\mathrm{m}}=0.67 \mathrm{~m} \cdot \mathrm{s}^{-1}$ and $\phi_{\text {in }}=0.75$, and; (2) $U_{\mathrm{m}}=0.56 \mathrm{~m} \cdot \mathrm{s}^{-1}$ and $\phi_{\text {in }}=0.75$.

Figure 24: Velocity profiles $\left(a_{1} \rightarrow a_{2}\right)$ and instantaneous images $\left(b_{1} \rightarrow b_{2}\right)$ for dispersed flows, at: (1) $U_{\mathrm{m}}=0.84 \mathrm{~m} \cdot \mathrm{s}^{-1}$ and $\phi_{\text {in }}=0.25$, and; (2) $U_{\mathrm{m}}=0.83 \mathrm{~m} \cdot \mathrm{s}^{-1}$ and $\phi_{\text {in }}=0.90$. 


\section{Tables}

Table 1: Table of test liquid physical properties.

\begin{tabular}{|lcc|}
\hline & Oil & Glycerol solution 80 wt.\% with 0.4 $\mathbf{~ m L}$ \\
& Exxsol D80 & of 5 wt.\% solution of Eosin Y per litre (L) \\
\hline Density $\left(\mathbf{k g} \cdot \mathbf{m}^{-3}\right)$ & 796 & 1,205 \\
\hline Viscosity (Pa $\cdot \mathbf{s})$ & $2.3 \times 10^{-3}$ & $47 \times 10^{-3}$ \\
\hline Refractive Index & 1.444 & 1.444 \\
\hline
\end{tabular}

Table 2: Categorisation of observed flow regimes.

\begin{tabular}{|ll|}
\hline Flow Regime Categories & Flow Regimes \\
\hline \multirow{2}{*}{ Stratified Flow } & Stratified flow \\
\cline { 2 - 2 } & Stratified flow with droplets \\
\hline \multirow{3}{*}{ Mixed Flow } & Oil droplet layer \\
\cline { 2 - 2 } & Glycerol solution droplet layer \\
\cline { 2 - 2 } & Three-layer flow \\
\hline Two-Layer, Dispersed Over/Under & Oil dispersion over glycerol solution flow \\
\cline { 2 - 2 } Continuous Flow & Oil flow over glycerol solution dispersion \\
\hline Dispersed Flow & Oil continuous dispersed flow \\
\cline { 2 - 2 } & Glycerol solution continuous dispersed flow \\
\hline
\end{tabular}

